Florida International University FIU Digital Commons

3-11-2010

\title{
Glycemic Control: Risk Factors, Quality of Life, Workforce Participation, and Mortality Among US Adults with Type 2 Diabetes
}

Evelyn P. Davila

Florida International University, miss_evelynda@yahoo.com

DOI: $10.25148 /$ etd.FI10041607

Follow this and additional works at: https://digitalcommons.fiu.edu/etd

Part of the Epidemiology Commons

\section{Recommended Citation}

Davila, Evelyn P., "Glycemic Control: Risk Factors, Quality of Life, Workforce Participation, and Mortality Among US Adults with Type 2 Diabetes" (2010). FIU Electronic Theses and Dissertations. 180.

https://digitalcommons.fiu.edu/etd/180 


\section{FLORIDA INTERNATIONAL UNIVERSITY}

Miami, Florida

GLYCEMIC CONTROL: RISK FACTORS, QUALITY OF LIFE, WORKFORCE PARTICIPATION, AND MORTALITY AMONG US ADULTS WITH TYPE 2 DIABETES

A dissertation submitted in partial fulfillment of the requirements for the degree of DOCTOR OF PHILOSOPHY in

PUBLIC HEALTH

by

Evelyn Patricia Davila

2010 
To: Dean Fernando M. Treviño

R.Stempel College of Public Health and Social Work

This dissertation, written by Evelyn Patricia Davila, and entitled Glycemic Control: Risk Factors, Quality of Life, Workforce Participation, and Mortality among US Adults with Type 2 Diabetes, having been approved in respect to style and intellectual content, is referred to you for judgment.

We have read this dissertation and recommend that it be approved.

Mary Jo Trepka

David J. Lee

Jai Parkash

Theophile Niyonsenga, Major Professor

Date of Defense: March 11, 2010

The dissertation of Evelyn Patricia Davila is approved.

Dean Fernando M. Treviño R.Stempel College of Public Health and Social Work

Interim Dean Kevin O'Shea

University Graduate School

Florida International University, 2010 


\section{DEDICATION}

In loving memory of my mother Norma E. Davila (1955-1997) 


\section{ACKNOWLEDGMENTS}

I would like to thank the members of my dissertation committee who provided a great deal of help and advice while being supportive and caring. I would also like to thank the expert consultants of my dissertation committee, Drs Lora E. Fleming and Hermes Florez, for the many hours they spent giving me feedback and knowledge on the topics of occupational health and diabetes. I wish to also thank Drs William G LeBlanc, Kristopher L. Arheart, and Frederick Zimmerman who helped me with data management and statistical issues. Finally I want to thank Dr Nasar Ahmed for giving me great guidance, friendship, and mentorship since the beginning of the doctoral program and including during the final stages of the dissertation. 


\section{ABSTRACT OF THE DISSERTATION \\ GLYCEMIC CONTROL: RISK FACTORS, QUALITY OF LIFE, WORKFORCE PARTICIPATION, AND MORTALITY AMONG US ADULTS WITH TYPE 2 DIABETES \\ by}

Evelyn Patricia Davila

Florida International University, 2010

Miami, Florida

Professor Theophile Niyonsenga, Major Professor

Despite research showing the benefits of glycemic control, it remains suboptimal among adults with diabetes in the United States. Possible reasons include unaddressed risk factors as well as lack of awareness of its immediate and long term consequences. The objectives of this study were to, using cross-sectional data, 1) ascertain the association between suboptimal (Hemoglobin A1c (HbA1c) $\geq 7 \%$ ), borderline (HbA1c 7 $8.9 \%$ ), and poor $(\mathrm{HbA} 1 \mathrm{c} \geq 9 \%$ ) glycemic control and potentially new risk factors (e.g. work characteristics), and 2) assess whether aspects of poor health and well-being such as poor health related quality of life (HRQOL), unemployment, and missed-work are associated with glycemic control; and 3) using prospective data, assess the relationship between mortality risk and glycemic control in US adults with type 2 diabetes. Data from the 1988-1994 and 1999-2004 National Health and Nutrition Examination Surveys were used. HbAlc values were used to create dichotomous glycemic control indicators. Binary logistic regression models were used to assess relationships between risk factors, employment status and glycemic control. Multinomial logistic regression analyses were 
conducted to assess relationships between glycemic control and HRQOL variables. Zeroinflated Poisson regression models were used to assess relationships between missed work days and glycemic control. Cox-proportional hazard models were used to assess effects of glycemic control on mortality risk. Using STATA software, analyses were weighted to account for complex survey design and non-response. Multivariable models adjusted for socio-demographics, body mass index, among other variables. Results revealed that being a farm worker and working over 40 hours/week were risk factors for suboptimal glycemic control. Having greater days of poor mental was associated with suboptimal, borderline, and poor glycemic control. Having greater days of inactivity was associated with poor glycemic control while having greater days of poor physical health was associated with borderline glycemic control. There were no statistically significant relationships between glycemic control, self-reported general health, employment, and missed work. Finally, having an $\mathrm{HbA} 1 \mathrm{c}$ value less than $6.5 \%$ was protective against mortality. The findings suggest that work-related factors are important in a person's ability to reach optimal diabetes management levels. Poor glycemic control appears to have significant detrimental effects on HRQOL. 


\section{TABLE OF CONTENTS}

CHAPTER

PAGE

I. INTRODUCTION 1

Overview 1

Research questions and hypotheses $\quad 5$

$\begin{array}{ll}\text { Significance of the present study } & 9\end{array}$

II. LITERATURE REVIEW 10

$\begin{array}{ll}\text { Overview } & 10\end{array}$

Risk factors of suboptimal and poor glycemic control $\quad 10$

Potentially new risk factors for suboptimal and poor glycemic control 11

$\begin{array}{ll}\text { Consequences of suboptimal and poor glycemic control } & 14\end{array}$

Diabetes, glycemic control and health related quality of life 15

Workforce participation, unemployment and absenteeism 16

$\begin{array}{ll}\text { Workforce participation and glycemic control } & 17\end{array}$

$\begin{array}{lr}\text { Glycemic control and mortality risk } & 18\end{array}$

III. METHODOLOGY 20

$\begin{array}{ll}\text { Overview } & 20\end{array}$

Sample and description of data sets $\quad 20$

$\begin{array}{ll}\text { Inclusion and exclusion criteria } & 22\end{array}$

Variables used for inclusion/exclusion criteria $\quad 22$

$\begin{array}{ll}\text { Outcome and predictor variables } & 23\end{array}$

$\begin{array}{ll}\text { Potential confounding variables } & 27\end{array}$

$\begin{array}{ll}\text { Sample weight variables } & 30\end{array}$

Primary sampling units (PSU) and strata variables $\quad 32$

Data management and preparation $\quad 33$

Missing data 33

Model criteria and strategy $\quad 34$

Interaction terms $\quad 36$

$\begin{array}{ll}\text { Statistical analyses } & 37\end{array}$

IV. RESULTS 45

Overview 45

$\begin{array}{ll}\text { General characteristics of study sample } & 47\end{array}$

Potentially new risk factors and glycemic control 52

$\begin{array}{ll}\text { Suboptimal glycemic control } & 58\end{array}$

Borderline glycemic control $\quad 61$

$\begin{array}{ll}\text { Poor glycemic control } & 64\end{array}$

$\begin{array}{ll}\text { Summary of results of research question } 1 & 67\end{array}$

The association between glycemic control and HRQOL 68

$\begin{array}{ll}\text { Self-rated general health } & 69\end{array}$ 
Days of poor mental health in the past 30 days $\quad 70$

Days of poor physical health in the past 30 days 75

Days of inactivity due to poor health in the past 30 days 77

$\begin{array}{ll}\text { Missed work days and glycemic control } & 79\end{array}$

$\begin{array}{ll}\text { Employment and glycemic control } & 79\end{array}$

Summary of results of research question $2 \quad 82$

Glycemic control and all-cause mortality risk $\quad 82$

Suboptimal glycemic control $\quad 86$

$\begin{array}{ll}\text { Borderline glycemic control } & 87\end{array}$

Poor glycemic control $\quad 88$

Glycemic control and cardiovascular mortality risk $\quad 90$

Suboptimal glycemic control $\quad 91$

Borderline glycemic control $\quad 92$

Poor glycemic control $\quad 93$

Summary of results of research question $3 \quad 94$

V. DISCUSSION 95

Overview 95

Potentially new risk factors and glycemic control 95

Glycemic control and work hours $\quad 96$

Glycemic control and farm workers $\quad 99$

Glycemic control and desire to lose weight 100

$\begin{array}{ll}\text { Glycemic control and secondhand smoke exposure } & 102\end{array}$

Glycemic control and health related quality of life 103

Glycemic control and self-reported general health, physical health,
and activity limitation

$\begin{array}{ll}\text { Glycemic control and mental health } & 107\end{array}$

Workforce participation and glycemic control 111

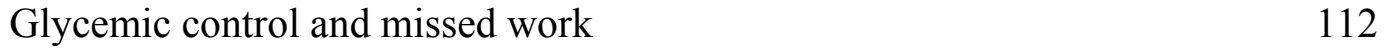

Glycemic control and unemployment 113

Mortality and glycemic control 115

$\begin{array}{ll}\text { Strengths and limitations } & 120\end{array}$

VI. CONCLUSION 126

Employers $\quad 129$

Individuals with diabetes and their healthcare providers 131

Public health advocates and professionals 133

$\begin{array}{ll}\text { Future research } & 134\end{array}$

LIST OF REFERENCES 136

$\begin{array}{ll}\text { VITA } & 161\end{array}$ 


\section{LIST OF TABLES}

TABLE

PAGE

1. List of key STATA commands used in present study

2a. Demographic characteristics among individuals classified with type 2 diabetes, 20+ years of age, NHANES III (1988-1994) and

NHANES 1999-2004

$2 \mathrm{~b}$. Presence of potentially new risk factors among individuals classified with type 2 diabetes, 20+ years of age, NHANES III (1988-1994) and NHANES 1999-2004

2c. Presence of glycemic control group characteristics among idividuals classified with type 2 diabetes, 20+ years of age, NHANES III (1988-1994) and NHANES 1999-2004

2d. Categorical health indicators and behaviors characteristics among Individuals classified with type 2 diabetes, 20+ years of age, NHANES III (1988-1994) and NHANES 1999-2004

2e. Numerical health indicators and behavioral characteristics among individuals classified with type 2 diabetes, 20+ years of age, NHANES III (1988-1994) and NHANES 1999-2004

3a. Cross-tabulations ${ }^{\dagger}$ of demographic variables with suboptimal $(\mathrm{HbA} 1 \mathrm{c} \geq 7.0 \%)$, borderline $(\mathrm{HbA} 1 \mathrm{c} \geq 7.0-8.9 \%)$, and poor $(\mathrm{HbA} 1 \mathrm{c} \geq$ 9.0\%) glycemic control, employed adults classified with type 2 diabetes, NHANES 1999-2004

3b. Cross-tabulations ${ }^{\dagger}$ of potentially new risk factors variables with suboptimal $(\mathrm{HbA} 1 \mathrm{c} \geq 7.0 \%)$, borderline $(\mathrm{HbA} 1 \mathrm{c} \geq 7.0-8.9 \%)$, and poor (HbA1c $\geq 9.0 \%$ ) glycemic control, employed adults classified with type 2 diabetes, NHANES 1999-2004

3c. Cross-tabulations ${ }^{\dagger}$ of health indicators and behaviors variables with suboptimal $(\mathrm{HbA} 1 \mathrm{c} \geq 7.0 \%)$, borderline (HbAlc $\geq 7.0-8.9 \%$ ), and poor (HbA1c $\geq 9.0 \%$ ) glycemic control, employed adults classified with type 2 diabetes, NHANES 1999-2004

3d. Mean comparisons for numerical variables with suboptimal $(\mathrm{HbA} 1 \mathrm{c} \geq$ $7.0 \%$ ), borderline (HbA1c $\geq 7.0-8.9 \%$ ), and poor (HbA1c $\geq 9.0 \%$ ) glycemic control, employed adults classified with type 2 diabetes, NHANES 19992004 
4a. Univariable logistic regression. Relationships between demographic characteristics with suboptimal (HbA1c $\geq 7.0 \%$ ), borderline (HbA1c $\geq 7.0$ $8.9 \%$ ), and poor ( $\mathrm{HbA} 1 \mathrm{c} \geq 9.0 \%$ ) glycemic control, employed adults classified with type 2 diabetes, NHANES 1999-2004

4b. Univariable logistic regression. Relationship between potentially new risk factors with suboptimal (HbAlc $\geq 7.0 \%)$, borderline $(\mathrm{HbAlc} \geq 7.0$ $8.9 \%$ ), and poor ( $\mathrm{HbA} 1 \mathrm{c} \geq 9.0 \%)$ glycemic control, employed adults classified with type 2 diabetes, NHANES 1999-2004

4c. Univariable logistic regression. Health indicators and behaviors factors associated suboptimal (HbA1c $\geq 7.0 \%)$, borderline (HbAlc $\geq 7.0-8.9 \%)$, and poor $(\mathrm{HbA} 1 \mathrm{c} \geq 9.0 \%)$ glycemic control, employed adults classified with type 2 diabetes, NHANES 1999-2004

5. Multivariable logistic regression. Relationship between suboptimal glycemic control and work characteristics among employed adults with type 2 diabetes, NHANES 1999-2004

6. Multivariable logistic regression. Relationship between borderline (HbA1c 7-8.9\%) glycemic control and work characteristics among employed adults with type 2 diabetes, NHANES 1999-2004

7. Multivariable logistic regression. Relationship between poor glycemic control $(\mathrm{HbA} 1 \mathrm{c} \geq 9.0 \%)$ and work characteristics among employed adults with type 2 diabetes, NHANES 1999-2004

8. Cross-tabulations ${ }^{\dagger}$ of health related quality of life variables and suboptimal (HbAlc $\geq 7.0 \%)$, borderline (HbA1c $\geq 7.0-8.9 \%)$, and poor (HbAlc $\geq 9.0 \%$ ) glycemic control, adults classified with type 2 diabetes, NHANES 1999-2004

9. Multinomial logistic regression. Relationship between days of poor physical and mental health and activity limitation among adults with type 2 diabetes with suboptimal (HbA $1 \mathrm{c} \geq 7.0 \%$ ), borderline (HbA1c $\geq 7.0$ $8.9 \%$ ), and poor (HbA1c $\geq 9.0 \%$ ) glycemic control, NHANES 2001-2004

10. Multinomial logistic regression. Relationship between suboptimal glycemic control ( $\mathrm{HbA} 1 \mathrm{c} \geq 7.0 \%)$ and days with poor mental health among adults with type 2 diabetes, NHANES 2001-2004

11. Multinomial logistic regression. Relationship between borderline glycemic control (HbA1c 7-8.9\%) and days with poor mental health among adults with type 2 diabetes, NHANES 2001-2004 
12. Multinomial logistic regression. Relationship between poor glycemic Control $(\mathrm{HbA} 1 \mathrm{c} \geq 9.0 \%)$ and days with poor mental health among adults with type 2 diabetes, NHANES 2001-2004

13. Multinomial logistic regression. Relationship between borderline glycemic control (HbAlc 7-8.9\%) and days with poor physical health among adults with type 2 diabetes, NHANES 2001-2004

14. Multinomial logistic regression. Relationship between poor glycemic control (HbA1c $\geq 9.0 \%)$ and inactivity days among adults with type 2 diabetes, NHANES 2001-2004

15. Cross-tabulations ${ }^{\dagger}$ of health related quality of life variables and suboptimal $(\mathrm{HbA} 1 \mathrm{c} \geq 7.0 \%)$, borderline $(\mathrm{HbA} 1 \mathrm{c} \geq 7.0-8.9 \%)$, and poor (HbA1c $\geq 9.0 \%$ ) glycemic control among adults classified with type 2 diabetes, NHANES 1999-2004

16. Multivariable logistic regression. Relationship between employment status and poor glycemic control (HbA1c $\geq 9 \%$ ) among adults with type 2 diabetes, NHANES 1999-2004

17. Cross-tabulations ${ }^{\dagger}$ of cause of death with participant characteristics, adults classified with type 2 diabetes, NHANES 1986-1994 and NHANES III mortality linked data file

18. Un-adjusted Cox proportional hazards model. Factors associated with all-cause and cardiovascular mortality risk among adults with type 2 diabetes, NHANES III

19. Adjusted Cox proportional hazards model. Relationship between suboptimal glycemic control and all-cause mortality risk among adults with type 2 diabetes, NHANES III

20. Adjusted Cox proportional hazards model. Relationship between borderline glycemic control and all-cause mortality risk among adults with type 2 diabetes, NHANES III

21. Adjusted Cox proportional hazards model. Relationship between poor glycemic control and all-cause mortality risk among adults with type 2 diabetes, NHANES III

22. Adjusted Cox proportional hazards model. Relationship between suboptimal glycemic control (HbA1c $\geq 7.0 \%)$ and CVD mortality risk among adults with type 2 diabetes, NHANES III 
23. Adjusted Cox proportional hazards model. Relationship between borderline glycemic control and CVD mortality risk among adults with type 2 diabetes, NHANES III

24. Adjusted Cox proportional hazards model. Relationship between poor glycemic control and CVD mortality risk among adults with type 2 diabetes, NHANES III 


\section{LIST OF ACRONYMS}

ACCORD

AACE

BMI

CDC

CI

CV

CVD

DCCT

BRFSS

HbA1c

HR

HRQOL

HS

ICD

QOL

NCHS

$\mathrm{NH}$

NHANES

OR

PA

PSU
Action to Control Cardiovascular Risk in Diabetes

American Association of Clinical Endocrinologists

Body Mass Index

Centers for Disease Control and Prevention

Confidence Interval

Coefficient of Variation

Cardiovascular Disease

Diabetes Control and Complications Trial

Behavior Risk Factor Surveillance System

Glycosylated Hemoglobin A1c

Hazard Ratio

Health Related Quality of Life

High School

International Classification of Diseases

Quality of Life

National Center for Health Statistics

Non-Hispanic

National Health and Nutrition Examination Surveys

Odds Ratio

Physical Activity

Primary Sampling Units 
SHS

US
Secondhand Smoke Exposure

United States 


\section{CHAPTER I.}

\section{INTRODUCTION}

\section{Overview}

Diabetes mellitus is one of the most serious chronic diseases in the United States (US) and in the world. In the US alone, diabetes affects an estimated 7.0\% (20.8 million) of the total population, and another 54 million are at risk. ${ }^{1}$ The prevalence of diabetes in the US is expected to rise in the future given the continued increase in the prevalence of associated risk factors such as older age, obesity, and minority race and ethnicity (e.g. Blacks and Hispanics). ${ }^{2}$ Projections have indicated that the prevalence of diabetes will increase to 366 million worldwide by the year $2030,{ }^{3}$ with 39 million in the US by the year $2050 .{ }^{4}$ In the US, the death rate for diabetes increased by $45 \%$ from 1970 to $2002,{ }^{5}$ and in 2006, diabetes was the sixth leading cause of death in the US. ${ }^{6}$ In addition to the high risk of mortality associated with diabetes, diabetes is also associated with great morbidity and disability. For example, among adults over 20 years of age, diabetes is the leading cause of blindness, leg amputations, and kidney disease. ${ }^{1,7}$ Consequently, both indirect (e.g. due to disability, unemployment, premature death) and direct (e.g. medical costs including hospitalizations and treatment) costs associated with diabetes are high, with the total costs estimated at about $\$ 174$ billion, of which $\$ 116$ billion are medical. ${ }^{7}$ In fact, the medical expenditures among those individuals with diabetes are estimated to be more than twice of those without. ${ }^{7}$ Therefore, the public health and economic burden associated with diabetes is large and is likely to increase.

Diabetes is generally diagnosed using a fasting plasma glucose test with a glucose result of $126 \mathrm{mg} / \mathrm{dl}$ after an eight-hour fast or by a two hour oral glucose tolerance test 
with a glucose result of $200 \mathrm{mg} / \mathrm{dl}$ or more. ${ }^{8}$ Diabetes is primarily categorized as type 1 or type 2 . Type 1 diabetes is the least common, accounting for less than $10 \%$ of all cases; it is caused by almost complete lack of insulin secretion, usually due to the autoimmune destruction of pancreatic beta cells. ${ }^{9}$ Type 2 diabetes is much more common accounting for $90-95 \%$ of cases. Type 2 diabetes is characterized by impaired insulin secretion that could arise due to insulin resistance and it is highly associated with obesity. ${ }^{9}$ Although diabetes can be diagnosed at any age, individuals diagnosed at an older age are usually diagnosed with type 2 diabetes. Regardless of the classification of diabetes, the outcome is the same, namely high levels of blood glucose or hyperglycemia due to a combination of an increase in insulin resistance, a decrease in beta cell function, and a decrease in insulin secretion by the pancreas. ${ }^{1}$

People can live with diabetes without major complications as long as it is well controlled and managed. The aim of diabetes management is to adequately maintain normal blood glucose levels (i.e. $70-130 \mathrm{mg} / \mathrm{dl}$ ) in order to prevent microvascular (e.g., retinopathy, neuropathy, nephropathy) and macrovascular (e.g., peripheral vascular or cardiovascular disease $[\mathrm{CVD}])$ complications. ${ }^{10}$ A key element in diabetes management is glycemic control, which may be indicated by a person's HbAlc. The hemoglobin A1C test measures the percent of glucose in the bloodstream attached to hemoglobin molecules in the previous two to three months, which is the lifespan of red blood cells. Thus, the more excess glucose in the bloodstream, the higher the percentage of hemoglobin molecules attached, the higher the HbAlc level. This test has been considered the gold standard for assessing glycemic control in diabetes care for the last 25 years. ${ }^{10,11}$ Although there are other methods of assessing glycemic control, the HbAlc 
test has been shown to be better than serum fructosamine tests because the latter only measures glycemic control in the last 2 weeks. ${ }^{12}$ Although continuous (daily) blood glucose monitoring would provide the most accurate evaluation of a person's average blood glucose levels, the HbA1c test is more practical, less costly, and more convenient than continuous blood glucose monitoring.

Several guidelines for glycemic control currently exist. The American Diabetes Association (ADA) treatment goals for glycemic control recommend $\mathrm{HbA1c}$ values of $<$ 7.0\%. ${ }^{9}$ The International Diabetes Federation and the American Association of Clinical Endocrinologists (AACE) recommend an HbAlc value of $<6.5 \%$. ${ }^{13}$ However, an HbA1c value of $<7.0 \%$ is not always realistic for individuals who are older and with several coexisting conditions and/or those facing terminal illness. ${ }^{14}$ Thus, two commonly used thresholds for glycemic control are a HbAlc value of $\geq 7 \%$, which is generally considered suboptimal glycemic control, and a $\mathrm{HbA1c}$ value of $\geq 9 \%$, which is an indicator of poor glycemic control and more often used as a target value for patients in worse health (e.g., greater comorbidity and/or terminally ill). ${ }^{15-18}$

Research has shown clear evidence of the benefits of good glycemic control in terms of diabetes-related complications. For example, it has been noted that a $1 \%$ decrease in the HbA1c value can lead to a $10 \%$ reduction in risk of coronary artery disease. ${ }^{19,20}$ Moreover, good glycemic control has been associated with fewer diabetic complications and better metabolic control. ${ }^{11}$ Studies have also shown that macrovascular and microvascular complications are related to poor glycemic control. For example, poor glycemic control has been associated with severe periodontitis, ${ }^{21}$ incidence of proteinuria and symptoms consistent with diabetic neuropathy, ${ }^{22}$ and elevated C- 
reactive protein, a marker of systemic inflammation and risk factor for cardiovascular disease. $^{23}$

Given the medical benefits of good glycemic control, strong efforts by public health advocates and the medical community have been made to improve glycemic control among adults with diabetes. For example, national diabetes programs, targeted at the general public, such as the National Diabetes Education Program have launched campaigns like the "Control the ABC's" campaign, which stresses the importance of the HbA1c test, in addition to frequent blood pressure and cholesterol check-ups. ${ }^{24}$ The Diabetes Quality Improvement Project is another diabetes program that has been developed in order to improve diabetes management. ${ }^{25}$ This program's mission is to develop a set of standardized performance and outcome measures in order to make equal comparisons of diabetes management health care plans across the US. ${ }^{25}$

Despite research showing the importance of lower HbA1c levels for preventing diabetic complications and despite public health efforts towards diabetes management, glycemic control remains a serious public health problem in the US. For example, in the 1980s and 1990s, only about $37 \%$ of US adults diagnosed with diabetes had HbA1c levels $<7 \%$ (i.e. optimal glycemic control). ${ }^{25}$ Others have noted that $18 \%$ of the US population with diabetes in the $1980 \mathrm{~s}$ and $1990 \mathrm{~s}$ had $\mathrm{HbA} 1 \mathrm{c}$ values of $>9.5 \% .{ }^{26}$ More recent data have shown slight improvements, yet still about $44 \%$ of the population with diabetes has an HbA1c value of suboptimal glycemic control. ${ }^{27}$

There are several possible reasons why adults with diabetes are not reaching optimal (i.e. HbA1c of $<7 \%$ ) or good (HbAlc of $<9 \%$ ) glycemic control. One of the reasons could be the presence of unknown risk factors for suboptimal or poor glycemic 
control that may be acting as barriers to proper diabetes management. Another reason could be the fact that information about the known morbidity outcomes does not promote a sense of urgency to the individuals with diabetes that would otherwise motivate them to adhere to their diabetes treatment regimen. Thus, it is important to assess potentially new risk factors for suboptimal, borderline, and poor glycemic control, as well as to learn about the consequences of suboptimal, borderline, and poor glycemic control other than those that are traditionally studied and reported (e.g., macrovascular and microvascuar complications).

The objectives of this study were to: 1) ascertain whether suboptimal, borderline, or poor glycemic control are associated with factors such as numbers of hours worked, type of occupation, secondhand smoke (SHS) exposure, and desire to lose weight in US adults with type 2 diabetes; 2) assess whether aspects of poor health and well-being such as poor quality of life, unemployment and missed work days are associated with suboptimal, borderline, and poor glycemic control in US adults with type 2 diabetes; and 3) assess the relationship between the risk of mortality and suboptimal, borderline, and poor glycemic control in US adults with type 2 diabetes.

\section{$\underline{\text { Research questions and hypotheses }}$}

This research used secondary, cross-sectional data from a nationally representative sample survey (1999-2004 NHANES) and a prospective study design (1988-1994 NHANES and its linked mortality file) to assess the relationship between suboptimal, borderline, and poor glycemic control and potentially four new risk factors i) number of hours worked per week, (ii) type of occupation, (iii) SHS exposure, and (iv) 
desire to lose weight. In addition, this study assessed the relationship between suboptimal, borderline, and poor glycemic control and employment status, absenteeism and mortality. NHANES is a series of population based surveys conducted by the Centers for Disease Control and Prevention (CDC) and designed to monitor several aspects of health, thereby providing a nationally representative picture of the health and nutritional status of the US population. ${ }^{28}$ A summary of the research questions and hypotheses for this study is shown in figure 1.

\section{Figure 1. A diagrammatic view of study hypotheses}

\section{Research Question 1:}

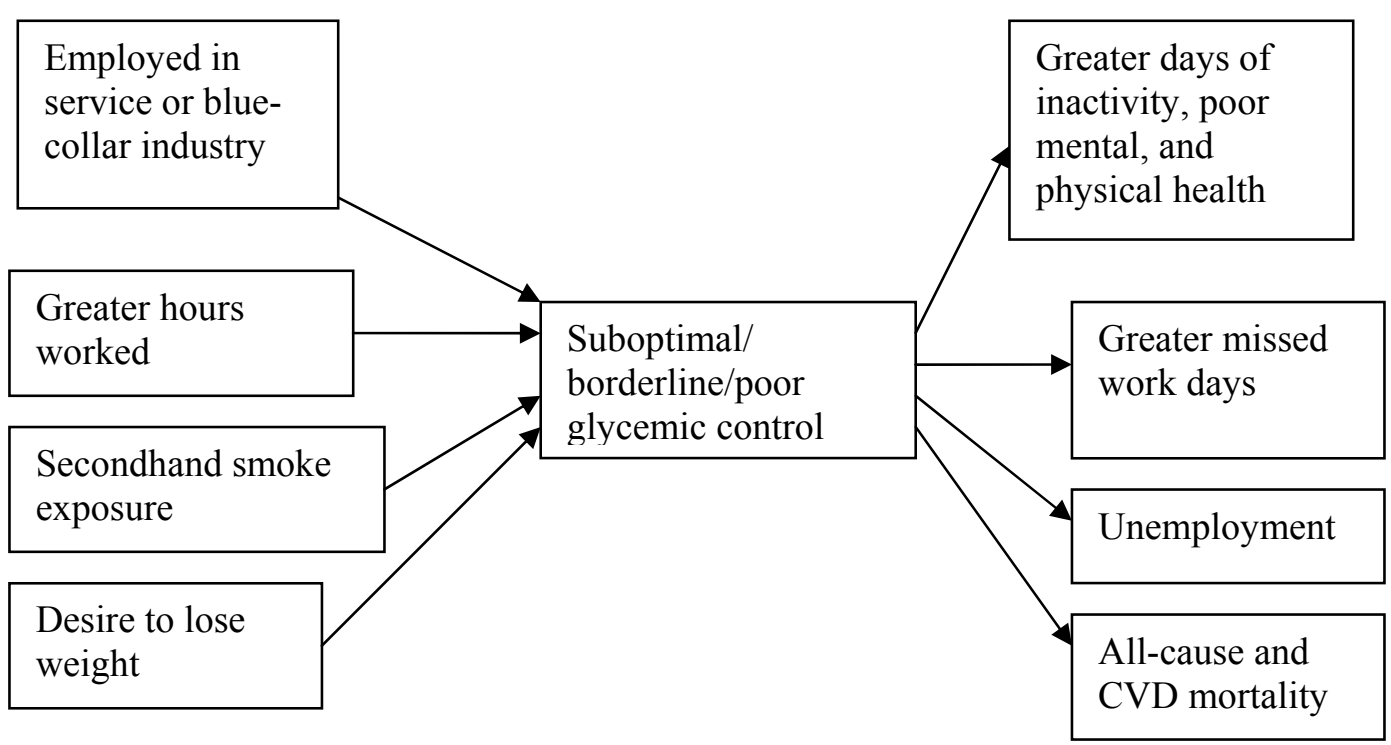

Research Question 1: Are suboptimal (HbA1c $\geq 7 \%$ ), borderline (HbA1c 7$8.9 \%)$, and poor $(\mathrm{HbA} 1 \mathrm{c} \geq 9 \%)$ glycemic control associated with potentially new risk factors such as i) number of hours worked per week, (ii) type of occupation, (iii) SHS 
exposure, and (iv) desire to lose weight among US adults with type 2 diabetes? This question leads to the following hypotheses.

Hypothesis 1a: Suboptimal, borderline, and poor glycemic control are associated with greater number of hours worked per week after controlling for potential confounders such as (i) duration of diagnosis, (ii) body mass index (BMI), (iii) alcohol use, (iv) greater number of non-CVD chronic conditions, (v) family history of diabetes, (vi) insurance status, (vii) sex, (viii) physical activity, (ix) diabetes treatment, and (x) race/ethnicity.

Hypothesis 1b: Suboptimal, borderline, and poor glycemic control are associated with occupational groups with greater time constraints or demands such as blue collar and service workers versus white collar workers after controlling for potential confounders such as (i) duration of diagnosis, (ii) BMI, (iii) alcohol use, (iv) greater number of non-CVD chronic conditions, (v) family history of diabetes, (vi) insurance status, (vii) sex, (viii) physical activity, (ix) diabetes treatment, and (x) race/ethnicity. Hypothesis 1c: Among self-reported non-smokers, suboptimal, borderline, and poor glycemic control are associated with being exposed to secondhand smoke after controlling for potential confounders such as (i) duration of diagnosis, (ii) BMI, (iii) alcohol use, (iv) greater number of non-CVD chronic conditions, (v) family history of diabetes, (vi) insurance status, (vii) sex, (viii) physical activity, (ix) diabetes treatment, and $(\mathrm{x})$ race/ethnicity.

Hypothesis 1d: Suboptimal, borderline, and poor glycemic control are associated with desire to lose weight after controlling for potential confounders such as (i) duration of diagnosis, (ii) BMI, (iii) alcohol use, (iv) greater number of non-CVD chronic 
conditions, (v) family history of diabetes, (vi) insurance status, (vii) sex, (viii) physical activity, (ix) diabetes treatment, and (x) race/ethnicity.

Research Question 2: Are a) health-related quality of life (HRQOL) measures indicative of poor health [i.e. (i) poor self-reported general health and greater number of days (ii) poor physical health, (iii) poor mental health and (iv) limited activity in the past 30 days]; b) greater days of missed work in the past 12 months (i.e. absenteeism), and c) unemployment, associated with suboptimal (HbA1c $\geq 7 \%)$, borderline (HbA1c 7-8.9\%), and poor (HbAlc $\geq 9 \%$ ) glycemic control among US adults with type 2 diabetes, after controlling for potential confounders (mentioned in hypothesis 1)? This research question leads to the following hypotheses:

Hypothesis 2a: All four HRQOL measures are associated with suboptimal, borderline, and poor glycemic control, after controlling for potential confounders.

Hypothesis $2 \mathrm{~b}$ : Greater number of missed work days in the past 12 months is associated with suboptimal, borderline, and poor glycemic control, after controlling for potential confounders.

Hypothesis 2c: Unemployment is associated with suboptimal, borderline, and poor glycemic controls, after controlling for potential confounders.

Research Question 3: Greater mortality risk among are associated with suboptimal (HbAlc $\geq 7 \%$ ), borderline (HbAlc 7-8.9\%), and poor (HbAlc $\geq 9 \%$ ) glycemic control among US adults with type 2 diabetes after controlling for potential confounders as noted in hypothesis 1 ? Two hypotheses are derived from this research question.

Hypothesis 3a: All-cause mortality risk is associated with suboptimal, borderline, and poor glycemic control, after controlling for potential confounders. 
Hypothesis 3b: CVD mortality risk is associated with suboptimal, borderline, and poor glycemic control, after controlling for potential confounders.

\section{$\underline{\text { Significance of the present study }}$}

Results from this study will provide information of public health significance for several reasons. First, by using a nationally representative sample of adults, this proposed study will allow generalization of the findings related to glycemic control among US adults with a self-reported diagnosis of diabetes. Second, by using a large nationally representative database, a comprehensive and thorough statistical assessment of the relationship between potentially new risk factors (e. g. type of occupation, hours worked, secondhand smoke exposure, and desire to lose weight) with suboptimal, borderline, and poor glycemic control, while adjusting for potential confounders, will be possible. Third, by studying the relationship between glycemic control and HRQOL, as well as employment and absenteeism, a better understanding of the overall well-being and the disability/dysfunction associated with poorly managed diabetes will be gained; thus, providing an estimate of the impact of disease that is not obtained in traditional morbidity

and mortality outcome measures. ${ }^{29}$ Fourth, by studying the association between glycemic control and mortality risk using prospective data, the long-term benefits due to good diabetes management will be elucidated. 


\section{CHAPTER II.}

\section{LITERATURE REVIEW}

\section{$\underline{\text { Overview }}$}

The literature search for this study included a comprehensive review of scientific articles in English available in databases such as MEDLINE using MeSH and keywords such as glycemic control, A1C, diabetes management, diabetes control, hemoglobin, diabetes treatment, among others from all years, as early as from 1950. In addition, articles that were found to be relevant from these searchers were used to capture additional articles by examining their reference lists as well as by looking at others who cited them. The literature review is presented separately for each research question and hypothesis.

\section{$\underline{\text { Risk factors of suboptimal and poor glycemic control }}$}

There have been several national and international studies assessing factors associated with poor glycemic control such as (i) minority race-ethnicity (e.g. Blacks and Hispanics), (ii) older age, (iii) male sex, (iv) drinking alcohol, (v) longer duration of diabetes, (vi) diabetes treatment (e.g., insulin or oral antidiabetic drugs), (vii) lack of insurance, (viii) having a CVD-related comorbidity (e.g. hypercholesterolemia, hypertension, microalbuminuria, kidney disease), (ix) positive family history of diabetes, BMI, smoking, (x) greater physical activity, (xi) lack of adherence to diabetes management, and (xii) certain non-CVD chronic conditions (such as depression). ${ }^{30-39}$ Though these traditionally studied factors are important in glycemic control, research has suggested that other factors such as work characteristics (e.g. an individual's occupation ${ }^{40}$ and the number of hours worked per week), ${ }^{41,42}$ secondhand smoke exposure, ${ }^{43}$ and the 
desire to lose weight may also influence glycemic control; yet the nature of the relationship between these factors and glycemic control has not been well studied.

Potentially new risk factors for suboptimal and poor glycemic control

Work constitutes an important part of most adult lives, and under several conditions can be the cause of the ill health. Thus, it is possible that glycemic control is also affected by work factors. However, few studies exist relating work characteristics with glycemic control among adults with diabetes.

The number of hours a person works is one factor that may be associated with poor glycemic control. Indeed, it is plausible that individuals working long hours are less likely to be able to properly manage their diabetes due to the lack of time to check their blood glucose levels, inject insulin, take oral agents when necessary, or eat well-balanced meals at regular time intervals, all factors that may affect glucose levels. Furthermore, research has shown that individuals with diabetes do consider their disease as an issue of concern affecting the type of jobs they seek, as well as the breaks and the work schedule they need. ${ }^{44,45}$ Although work-hours alone have not been linked to glycemic control, a related variable, job stress, has been associated with greater HbAlc levels even among employed individuals without diabetes in non-US studies. ${ }^{42,46,47}$ Job stress, a term used to describe the psychological and physiological effects resulting from being employed in a high pressure and pace job coupled with lack of workplace decision or control, has even been associated with other cardiovascular risk factors. ${ }^{48-51}$

Work characteristics including the length of and control over work schedules may also affect glycemic control. In fact, commercial truck drivers reported problems 
regulating their glucose levels and have blamed their unhealthy dietary patterns due to being on the road. ${ }^{52}$ In relation to diabetes, certain occupations that are characterized as having high work demands (e.g. long work hours with limited breaks) have been shown to have a higher prevalence of diabetes. ${ }^{53}$ For example, in a study of industrial factory workers in Japan, Japanese transport workers (e.g. those picking up and delivering factory materials) were shown to have greater risk of developing type 2 diabetes, compared to Japanese managers, clerical workers, and technical workers, even after adjusting for obesity and other potential confounders. ${ }^{54}$ Industrial male workers and female nurses have also been shown to have greater risk of type 2 diabetes. ${ }^{41,55}$ Nonetheless, office workers have also been shown to be at lower risk for incidence of diabetes. ${ }^{56}$ In the only known study relating occupation to glycemic control, which was based on data of adults with diabetes in New Zealand, the authors showed that glycemic control, as measured by fructosamine levels, did not vary by type of occupation (i.e. no difference found among administrative, clerical/sales/service, skilled trades, and unskilled manual workers). ${ }^{57}$ Given the few studies relating occupation to glycemic control diabetes, ${ }^{41,54-57}$ more research is needed to determine the association between glycemic control and occupational factors.

Another factor that has not been fully investigated but is gaining recognition as a major determinant of several chronic conditions is passive smoking, also known as SHS exposure or environmental tobacco smoke. Aside from its effects on lung and respiratory functions, SHS exposure has been associated with greater acute coronary syndromes and an increase in inflammatory markers. ${ }^{58}$ Research has shown that there is an association between diabetes and smoking. ${ }^{59}$ Specifically, studies have shown that smoking 
contributes to worse metabolic control among individuals with diabetes, ${ }^{60}$ greater likelihood of both microvascular ${ }^{61}$ and macrovascular complications,${ }^{62}$ and worse glycemic control. ${ }^{63,64}$ There have been fewer studies on the relationship between SHS and diabetes. ${ }^{43,65}$ Nevertheless, these studies have shown a relationship between SHS and glucose intolerance among young adults ${ }^{43}$ as well as incidence of diabetes. ${ }^{65}$ To date, there are very few if any studies, to the author's knowledge based on a robust MEDLINE search of published articles since 1950, investigating the effects of SHS and glycemic control.

Another potential risk factor for suboptimal and poor glycemic control is an individual's desire to lose weight or control their weight. For example, it is possible that some adults with diabetes who want to lose or control their weight avoid taking insulin as recommended by their physician knowing that weight gain is one of the potential side effects of insulin use. ${ }^{66-69}$ Thus, not taking insulin when needed may result in poor glycemic control, particularly if the individual has other unhealthy dietary and eating behaviors. In fact, poor glycemic control has been found among adolescents and young adult females with eating disorders ${ }^{70-72}$ and among adolescents that perceived themselves as being overweight. ${ }^{73}$ Furthermore, not taking insulin in order to avoid weight gain is actually considered one of the DSM-IV diagnostic criteria for bulimia. ${ }^{72}$ However, there are very few if any studies specifically assessing the relationship between the desire to lose weight and glycemic control among US adults with diabetes. 


\section{Consequences of suboptimal and poor glycemic control}

Increasing the years of healthy life and improving the quality of life (QOL) are some of the overarching goals of Healthy People 2010. ${ }^{74}$ Although the term QOL is mostly used by the social sciences, in public health and the medical professions, the focus has been placed on the concept of Health Related Quality of life (HRQOL). The CDC defines HRQOL as an individual's "perceived physical and mental health over time", a measure which provides a better insight into how an illness or condition can affect everyday life. ${ }^{75}$ In general, HRQOL indicators can be thought of as a way to assess overall disability and poor function resulting from a disease or condition, aspects that may not be determined when looking at traditional measures of morbidity and mortality. ${ }^{76}$ In addition, HRQOL measures can be especially useful when assessing the long term consequences of chronic conditions since poor disease management can take years to develop as a clinical or medical outcome,${ }^{77}$ impairing a person's overall health status and well-being, particularly one's mental health.

Since the 1990s, there has been growing interest in HRQOL, partly due to the growth of the US aging and chronically-ill population. ${ }^{76}$ Consequently, the CDC developed four "core" HRQOL questions, also referred to as "Core Healthy Day" measures, which were first included in the Behavioral Risk Factor Surveillance System (BRFSS) in 1993, and were then included in the National Health and Nutrition Examination Surveys (NHANES) beginning with the 2001-2002 cycle. These four questions measure: 1) overall self-rated general health [fair/poor versus excellent/very good/good]; 2) physical health based on the number of days with poor physical health in the past 30 days; 3 ) mental health based on the number of days with poor mental and 
emotional health in the past 30 days; and 4) disability and loss in productivity based on the number of days with activity limitation in the past 30 days. These core HRQOL questions have been found to have an acceptable validity and reliability when compared to the Medical Outcomes Short-Form 36 (SF-36) ${ }^{78}$ The SF-36 is a validated instrument with 36 questions about perceived health status and number of disability days, and is considered the gold standard for evaluating overall health including physical and mental health. ${ }^{78}$

\section{Diabetes, glycemic control and health related quality of life}

Individuals with diabetes have been shown to have poor quality of life. ${ }^{79}$ One of the reasons for such poor quality of life could be the increased number of daily medical (or health) responsibilities adults diagnosed with diabetes have. The constant reminder of the importance and necessity of responsibilities and activities such as taking insulin or oral diabetic medication at regular time intervals (e.g. after consuming certain meals or performing various physical activities) can take its toll in a person's life. ${ }^{79}$ In fact, because of the potential effects on HRQOL, it is believed that some physicians delay the initiation of insulin treatment, although a study showed that initiation of insulin treatment does not necessarily alter quality of life in a negative way. ${ }^{80}$ Nevertheless, it is possible that adults with diabetes have poor quality of life because of the health complications related to poorly managed diabetes, such as retinopathy, neuropathy, and cardiovascular conditions. ${ }^{77,81,82}$ Furthermore, given that fewer diabetic complications are found among those with better glycemic control, HRQOL is likely to be associated with glycemic control. However, the effect of glycemic control on HRQOL among diabetics is not clear. 
Indeed, while some studies have found poor glycemic control to be associated with poor quality of life, ${ }^{83-85}$ other studies have not found any association. ${ }^{86-89}$ For example, functional disability, which is a component of HRQOL, has shown an inconsistent relationship with glycemic control among adults with diabetes. ${ }^{90,91}$

None of the studies relating QOL and glycemic control were based on a nationally representative data of adults with diabetes. In addition, the discrepancies in the results of these studies could be a function of the different QOL measurement instruments employed. For example, while some studies have used what are known as "generic" QOL measures such as those developed and used by the CDC, others have used more "illness oriented" measures that are developed specifically for health conditions such as diabetes and include the Diabetes Qualify of Life Measure, the Diabetes Treatment Satisfaction Questionnaire, and the Problem Areas in Diabetes. ${ }^{77,79}$ Thus, there is a need of nationally representative studies aimed at understanding the association between poor glycemic control and HRQOL among US adults with diabetes using validated measures.

Workforce participation: unemployment and absenteeism

As the US population continues to change, so does the US workforce. Specifically, the workforce is expected to become increasingly diverse with growing number of elderly and minority workers. In fact, Hispanics are projected to represent $17 \%$ of the workforce by 2020 , an increase of $11 \%$ from the 1980 s. $^{40}$ Moreover, by the year 2012, there will be over 40 million American workers aged 65 and older. ${ }^{92}$ Since the elderly, racial and ethnic subgroups such as Hispanics and Blacks are known to have a higher prevalence of many chronic health conditions such as diabetes, ${ }^{93}$ the number of 
workers with these chronic conditions is expected to steadily increase. The consequences of these increases are likely to adversely impact workplace productivity, resulting in economic losses not only to the individual or the employer, but also to the nation as a whole. ${ }^{94}$ Furthermore, a person's employment status, and consequently economic status, may directly influence their access to health care and their ability to pay for medications or treatment requirements, which in turn affect proper management of the disease, and therefore may worsen patient prognosis. In addition to employment status, the number of missed work days is another individual-level measure for monitoring the impact of chronic disease on worker productivity. Missed work or "absenteeism" may provide a reflection of the disability that is experienced by diabetics with poor health and wellbeing that may perhaps not be obtainable by employment statistics. ${ }^{95,96}$ The disability and consequently diminished productivity at work may be of particular concern for employers given the financial implications such as decreased productivity.

\section{Workforce participation and glycemic control}

There are various studies assessing the relationship between diabetes status and workforce participation. For example, studies have shown that diabetics are both less

likely to be represented in the workforce and more likely to miss work, ${ }^{57,97-99}$ particularly among those individuals with more severe diabetic complications. ${ }^{94,100,101}$ What makes some adults with diabetes more likely to miss work or be unemployed versus other adults with diabetes is not entirely clear. However, one of the reasons could be a person's level of glycemic control. 
There are few studies assessing the relationship between glycemic control and workforce participation. In a double-blind randomized controlled trial of adults with type 2 diabetes, the treatment group with more strict diabetes treatment regimens (e.g. lower HbAlc target) had a more favorable outcome in terms of retained employment and less absenteeism. ${ }^{102}$ In a cross-sectional study of diabetic patients from Michigan, having more hours of missed work in the past month was associated with poor glycemic control. ${ }^{103}$ However, in a study of adults with diabetes referred to a multi-center diabetes program in the US, there appeared to be no significant relationship between glycemic control and the risk of days lost from school or work even after controlling for confounders. ${ }^{104}$ Thus, the association between glycemic control and missed work days requires further study.

\section{$\underline{\text { Glycemic control and mortality risk }}$}

Along with improved quality of life and reduction in health disparity, increased life expectancy is a major goal of Healthy People 2010. Most studies that have assessed the relationship between glycemic control and mortality have been based on international studies, adults without diabetes, and/or chronically-ill populations. These studies have found that greater mortality risk is associated with poor glycemic control. For example, greater mortality risk among advanced chronic kidney disease and dialysis patients with

poor glycemic control has been observed. ${ }^{105-107}$ In a prospective study of individuals in the United Kingdom to assess the relationship between $\mathrm{HbAlc}$ levels and all-cause and CVD mortality (after a 6 year follow-up), it was shown that the all-cause and CVD mortality risk increased with greater HbA1c levels. ${ }^{108}$ 
Furthermore, most of the studies investigating the relationship between diabetes management measures (e.g., fasting blood glucose, HbAlc) and CVD events, including mortality risk, have been primarily of men. ${ }^{108}$ The few studies that do exist among women have suggested differences in risk for CVD events and/or mortality, with most of them noting a lower mortality risk among women. ${ }^{108-110}$ Some studies have even found a counterintuitive relationship between $\mathrm{HbAlc}$ and mortality, suggesting that strict diabetic treatment regimes may eventually be more harmful than beneficial in certain populations such as those with greater comorbidity. ${ }^{111,112}$ Thus, more research is needed to determine the association between glycemic control and mortality risk among US adults with diabetes. 


\section{CHAPTER III.}

\section{METHODOLOGY}

\section{Overview}

This study used cross-sectional secondary data from a nationally representative survey sample (1988-1994 NHANES [also known as NHANES III] and 1999-2004 NHANES) to assess the relationship between four potentially new risk factors (type of occupation, number of hours worked per week, SHS exposure, and desire to lose weight) and suboptimal, borderline, and poor glycemic control. In addition, this study estimated the association between suboptimal, borderline, and poor glycemic control and employment status, missed work, and mortality.

\section{$\underline{\text { Sample and description of data sets }}$}

The National Health and Nutrition Examination Survey (NHANES) is a health survey developed by the National Center for Health Statistics (NCHS) to gather information about the health status of the US population. NHANES uses a stratified, multi-stage complex probability design that allows for a nationally representative estimation of the non-institutionalized US population. ${ }^{28}$

Briefly, the sampling scheme in NHANES consists of four stages. The first stage involves selecting primary sampling units (PSU) from the entire nation. These are approximately the size of a large county or several small ones. The second stage involves the PSU units being divided in to small sections, usually about the size of city blocks. The third stage consists of selecting households at random within each of the sectors. Finally, stage four is where individuals are chosen from selected households, and 
selection is based on age-sex-race screening strata. Weights are based on the probability of a person being selected at each of these stages. Sampling weights are created to provide unbiased national estimates, and are adjusted for non-response and then poststratified to match with the US population census counts of subpopulations. ${ }^{28}$

NHANES data are collected by a trained team of professionals through a series of in-person interviews and medical examinations conducted at mobile examination clinics or at home. All NHANES data are de-identified, with only sequential numbers identifying each participant in order to protect participant confidentiality. Most NHANES data files are public-use files and the NHANES files used in this study were all publicuse files that were downloaded from the NCHS website. The NHANES III linked Mortality File used in this study was the public-use file that contains data of the major causes of death (i.e. not specific cause of death) from the National Death Index, with follow-up through December 31, 2000. ${ }^{113}$ Depending on the hypotheses being tested in this study, different NHANES survey years were used. The reason for this is because not all variables were available in all survey years. In addition, depending on the sampling methodology, NCHS does not recommend merging of the older NHANES (i.e. 19881994) with the more recent NHANES survey years. NHANES 1999-2004 was used for hypotheses 1, 2b, and 2c for utilized. NHANES 2001-2004 was used for hypothesis 2a, while NHANES 1988-1994 and its Mortality file for hypothesis 3a and 3b was used. 


\section{$\underline{\text { Inclusion and exclusion criteria }}$}

Individuals with a self-report of diabetes, aged 20 years or older, and not pregnant were included in the study. Individuals defined as having type 1 diabetes were excluded; the remaining sample was considered to have type 2 diabetes, as defined in previous studies. $^{31,36,40}$

Variables used for inclusion/exclusion criteria:

Self-report of diabetes: This was a categorical variable defined as: 1) yes and 2) no, based to the question "Other than during pregnancy, have you been ever been told by a physician that you have diabetes or sugar diabetes?" Participants who were diagnosed with diabetes before the age of 30 (see variable question below), and were treated with insulin only were further classified as having type 1 diabetes. This definition is consistent with that used in previous studies utilizing NHANES data and is due to the agreement within clinicians that an individual diagnosed with diabetes before the age of 30 is more likely to have type 1 diabetes, but since type 2 diabetes is becoming more prevalent among youth, taking insulin only is likely a result of having type 1 diabetes. ${ }^{31,36,40}$ Those individuals considered not to have type 1 diabetes, were classified as having type 2 diabetes, a definition consistent with previous NHANES analyses. ${ }^{31,36,40}$ Age at diabetes diagnosis: This was a continuous variable expressed in years based on the question "How old were you when a doctor or health care professional told you that you had diabetes or sugar diabetes?" and asked among all individuals that selfreported being diagnosed with diabetes. 


\section{Outcome and predictor variables:}

Glycemic control: the HbAlc value was a continuous variable expressed as a percentage of glucose in the bloodstream that is attached to hemoglobin molecules.

Suboptimal and poor glycemic control: An individual with a HbAlc value equal to or greater than $7 \%$ was said to have suboptimal glycemic control. On the other hand, an individual with a $\mathrm{HbA} 1 \mathrm{c}$ value equal to or greater than $9 \%$ was said to have poor glycemic control. To be consistent with many of the studies using glycemic control variables, suboptimal glycemic control was defined as: 1) yes, if $\mathrm{HbAlc} \geq 7 \%$, and 2) no, if HbA1c $<7 \%$ (reference group); while poor glycemic control was defined as: 1) yes, if $\mathrm{HbAlc} \geq 9 \%$, and 2) no, if HbAlc $<9 \%$ (reference group).

Since a response of "yes" to the suboptimal glycemic control variable actually includes individuals with poor glycemic control and there are clinical differences or manifestations between suboptimal and poor glycemic control, a third glycemic control variable was also created, labeled "borderline" glycemic control, for the purposes of this study. This variable was defined as: 1) yes if $\mathrm{HbA1c} \geq 7 \%$ but $\mathrm{HbA} 1 \mathrm{c}<9 \%$, and 2) no, if $\mathrm{HbA} 1 \mathrm{c}<7 \%$ (reference group); thus, for this variable all individuals with $\mathrm{HbA} 1 \mathrm{c} \geq 9 \%$ were excluded from the analyses. All analyses were repeated for this new glycemic control variable.

In the 1999-2004 NHANES, glycosylated hemoglobin in whole blood was measured by the Diabetes Diagnostic Laboratory at the University of Missouri-Columbia using Primus CLC330 and Primus CLC 385 (Primus Corporation, Kansas City, MO) with a high performance liquid chromatography system among all participants age 12 years of age or older. ${ }^{114}$ Several quality control procedures were taken to ensure accuracy 
of measures. These measures included verifying and repeating plasma (or blood) specimens of $\mathrm{HbAlc}$ that were above $14 \%$ or below $4 \%$ (the accepted range for $\mathrm{HbAlc}$ measures were from $2 \%$ to $20 \%) .{ }^{114}$ From the measurements that were repeated, a few were selected at random and the coefficient of variation (CV) calculated. The CV provides an estimate of the amount of dispersion in the measure, in other words, how much one measure differs from the second measure taken, with a lower CV indicating lower dispersion and less measurement error. The $\mathrm{CV}$ for these specimens ranged from 1.53 to 1.29 , which indicates adequate similarity in the specimens when repeated. ${ }^{114}$

Health related quality of life: HRQOL was based on four different variables: selfrated general health, number of days of poor mental health, days of poor physical health, and days of activity limitation. Each HRQOL measure, except for the general health variable, was categorized into three levels in order to be consistent with previous studies while the general health variable was dichotomized. ${ }^{115,116}$

General health: General health was a categorical variable based on the question "Would you say your health in general is fair, poor, good, very good, and excellent?" It was recoded to include only two categories: 1) fair/poor and 2) good/very good/excellent (reference group).

Days with poor mental health: This was a discrete variable expressed in days and based on the question "Now thinking about your mental health, which includes stress, depression, and problems with emotions, for how many days during the past 30 days was your mental health not good?." This variable was recoded into a categorical variable as follows: 1) $\geq 14$ days, 2) 1-13 days, and 3) none (reference group).

Days with poor physical health: This was a discrete variable expressed as days, 
and based on the question "Now thinking about your physical health, which includes physical illness and injury, for how many days during the past 30 days was your physical health not good?." For purpose of the analyses, this variable was redefined into a categorical variable as follows: 1) $\geq 14$ days, 2) 1-13 days, 3) none (reference group). Days with limited activity: This was a discrete variable expressed as days, and based on the question "During the past 30 days, for about how many days did poor physical or mental health keep you from doing your usual activities, such as self-care, work, school or recreation?." For the analyses, this variable was redefined into a categorical variable as follows: 1) $\geq 14$ days, 2) 1-13 days, and 3) none (reference group). All-causes and CVD mortality: The all-cause mortality variable was defined consistent with NCHS standards. Among participants in the NHANES III file, those that were found in the NHANES III Linked Mortality public-use file were considered dead, but if the person was not found in the file than the person was assumed alive. ${ }^{113,117}$ Briefly, this linkage was conducted by the NCHS and was done by a probabilistic match of information from participants aged 17 years or older in NHANES III with information from the National Death Index through December 31, 2000 to determine mortality status. More detail about the matching on mortality conducted by NCHS can be found elsewhere. ${ }^{113,117}$ Verification of correct matches was also conducted by NCHS staff. The underlying causes of death were based on the International Classification of Diseases (ICD), $9^{\text {th }}$ and $10^{\text {th }}$ Revision Clinical Modification and were grouped by the NCHS staff into a standard list of 113 death categories. The CVD mortality variable was defined based on the ICD-10 variable for CVD mortality, and coded as: 1) yes, and 2) no. The yes category corresponds to ICD-10 deaths codes: I10-I13, I20-I25, I44-I49, I50, I60-I69, and 
I70-I78. ${ }^{113,117}$ The no category corresponds to anyone who died from another cause. Person-years of follow-up is included as a variable in the NHANES III linked mortality data file and were calculated by NCHS staff by subtracting the NHANES III examination date (as listed in the NHANES III data file) from the date of follow-up (when the death status was ascertained).

Desire to lose weight: This was a categorical variable based on the question "Would you like to weigh less, about the same, or more." For the purposes of the present analyses, this variable was defined as: 1) less, and 2) about the same or more (reference group).

Employment status: This was a categorical variable based on the question "Did you work last week (at a job or business)?" This variable was defined as: 1) yes, and 2) no (reference group).

Numbers of hours worked: This was a numeric variable expressed in hours, based on the question "How many hours did you work last week at all jobs or businesses?" Along with using this variable as a numeric variable, it was also categorized into three categories: 1) 1-20 hours (to include those with part-time or less), 2) 21-40 (to include those with more than a part-time and inclusive of full-time, and 3) 41+ (to include those considered generally to be working overtime). These categories were partially based on the definitions of part-time, full-time, and over-time employment based on federal labor laws, particularly over-time work which is defined as more than 40 hours by the Bureau of Labor Statistics. ${ }^{118,119}$

Number of missed work days (absenteeism): This was a variable expressed in days based on the question "During the past 12 months, about how many days did you miss 
work at a job or business because of an illness or injury (do not include maternity leave)? Type of occupation: This was a categorical variable based on the NCHS industry codes. ${ }^{120}$ This variable was collapsed into four NCHS occupational groups: 1) white collar, 2) blue collar, 3) service, and 4) farm worker, as done in previous studies. ${ }^{121}$

Smoking status: This was a categorical variable created from several variables to have the following categories: 1) non-smoker with undetectable cotinine (reference group), 2) non-smoker with detectable cotinine, and 3) smoker. For this study, smoking status was defined as a combination of self-report and serum cotinine levels. Current smokers were participants who answered yes to the question "Do you smoke cigarettes now?", and/or with serum cotinine levels $>15 \mathrm{ng} / \mathrm{mL} .{ }^{122}$ Participants who answered yes to the question "Does anyone who lives [with you] smoke cigarettes, cigars, or pipes anywhere inside this home?" and/or had had a serum cotinine level at or above the level of detection but below $\leq 15 \mathrm{ng} / \mathrm{ml}$, and reported not being current smokers were categorized as being exposed to secondhand smoke. Non-exposed non-smokers were participants who reported being former smokers or never smoking, no reported home secondhand smoke, and had cotinine levels below the detection limit. The detection limit for NHANES III and NHANES 1999-2000 surveys was $0.035 \mathrm{ng} / \mathrm{ml}$, while for NHANES $2001-2004$ it was $0.015 \mathrm{ng} / \mathrm{ml}^{123,124}$

\section{$\underline{\text { Potential confounding variables }}$}

Confounding variables that were tested included the following:

$\underline{\text { Sex }}$ was a categorical variable defined as: 1) female (reference group), 2) male. Age group in years was a continuous variable that was also categorized as 
follows: 1) 20-44 (reference group), 2) 45-64, and 3) 65 or more. Most analyses used the categorized variable in order to be consistent with the other continuous variables that were categorized, such as body mass index.

$\underline{\text { Racelethnicity }}$ was a categorical variable defined as: 1) non-Hispanic White (reference group), 2) non-Hispanic Black, 3) Hispanic, and 4) other.

Educational level was a categorical variable defined as: 1) less than high school (reference group), 2) high school graduate or equivalent, and 3) > high school.

Marital status was a categorical variable defined as: 1) not married (reference group), and 2) married/with partner. The not married category included responses of single, divorced, and widowed, while the married category included living with partner.

Alcohol consumer was a categorical variable created based on the question "In your entire life did you drink at least 12 drinks of any type of alcoholic beverage?". It was defined as: 1) no, and 2) yes. This question was the only question related to alcohol that was used because of the large number of missing data for the other alcohol-related questions that exist in the NHANES data files.

Insurance status was a categorical variable defined as: 1) uninsured (reference group), and 2) insured.

Physical activity (PA) level was a categorical variable defined as 1) vigorous, 2) moderate, and 3) none (reference group). "Vigorous" was a response of yes to the question: "Over the past 30 days did you do any vigorous PA for at least 10 minutes that caused heavy sweating, or large increases in breathing or heart rate?. "Moderate" was a response of yes to the question "Over the past 30 days did you do any moderate PA for at least 10 minutes that caused heavy sweating, or large increases in breathing or heart 
rate?" but no to the question of vigorous physical activity. A response of "no" to both the vigorous or moderate physical activity questions was defined as none. [Note: Although NHANES has other questions related to PA, these were the ones that where consistently asked from 1999-2004 and contained the least missing data.

Measured BMI group $\left(\mathrm{kg} / \mathrm{m}^{2}\right)$ was continuous variable that was redefined into a categorical variable as: 1) $\mathrm{BMI}<25$ (reference group), 2) BMI 25-29.9, and 3) $\mathrm{BMI} \geq 30$.

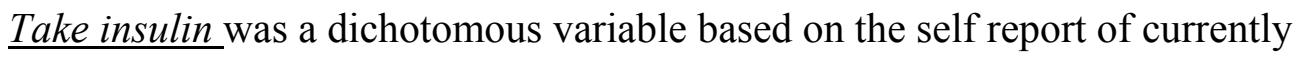
taking insulin defined as: 1) yes take insulin, and 2) do not take insulin (reference group).

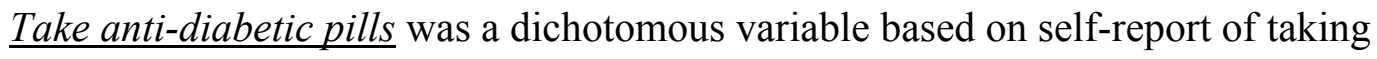
diabetic pills defined as: 1) yes take diabetic pills, and 2) do not take diabetic pills (reference group).

Duration of diabetes was a numeric variable expressed in number of years based on the variable for age of diabetes diagnosis and the variable for age.

Hypertension diagnosis was a categorical variable defined as: 1) no (reference), and 2) yes.

Total cholesterol (milligrams $(\mathrm{mg}) /$ deciliter $(\mathrm{dl})$ was a continuous variable expressed in $\mathrm{mg} / \mathrm{dl}$.

Albumin/creatinine ratio was a continuous variable based on a formula using two other variables: urinary albumin divided by urinary creatinine.

Percent (\%) carbs in diet was a continuous variable representing the percent of calories in the diet that came from carbohydrates and was based on a formula using two separate questions in the nutrition file of NHANES: the number of total calories in the diet and the number of calories from carbohydrates consumed (derived based on grams of 
carbohydrates consumed in the previous day; a 24 hour diet recall).

Cancer history was a created as a dichotomous variable based on the following two questions: "Has a doctor ever told you had skin cancer?" and "Has a doctor ever told you had other cancer?" A response of yes to either of the questions was defined as: 1) yes, while a response of no to both question was defined as 2) no (reference group).

History of stroke or heart failure was a dichotomous variable based on the following two questions: "Has a doctor ever told you had a stroke" and "Has a doctor ever told you had congestive heart failure." A response of yes to either question was defined as 1) yes, while a response of no to both questions was defined as 2) no (reference group).

Presence of asthma was a dichotomous variable based on the questions: "has a doctor ever told you had asthma" and "do you still have asthma?" A response of yes to both was defined as 1) yes, while a response of no to these two questions was defined as 2) no (reference group).

\section{Sample weight variables}

Each sampled person has a sampling weight providing unbiased national estimates. NHANES surveys use an unequal selection probability design. It is particularly important to know the weights when analyzing NHANES data because the NHANES oversamples some subgroups that may be of greater public health interest (e.g. African Americans). Weights are created by calculating a base weight (the reciprocal of the final probability of selection at each stage), then adjusting for non-response, and finally adjusting for post-stratification. ${ }^{28}$ 
Sample weight variables are calculated by the NCHS in two-year increments. For the continuous NHANES 1999-2002, a sample weight variable was created by NCHS (based on population estimates by the Bureau of the Census before the 2000 Decennial Census counts prior to public release), and can be obtained from the demographic data files for both cycles. ${ }^{28}$ The variable name for the weight variable depends on the type of data that are being investigated in the analysis and what data exists for the individuals in the study. Only one weight variable is typed in the command line in the STATA software for the analyses, and this weight variable is the one for the data file with a smaller sample size. For example, the questionnaire data files are those that have data on questions asked to participants while the laboratory files are those files that have data on blood or urine samples drawn from participants. Since it is more feasible and inexpensive to ask only questions in person rather than collect urine and blood samples, not all individuals have laboratory data. If one is interested in data from the laboratory file and an individual exist in both the questionnaire and laboratory files, then the weight variable for the laboratory file would be used.

As detailed in the NCHS documentation, ${ }^{28}$ each survey participant has several weights given differences in overall response rates to different survey components (e.g. home interview versus physical examination or laboratory specimen collection) as well as differences in population estimates provided from the Bureau of the Census for each given year. When using the NHANES III data file, we used the NCHS weight variable labeled wtpfhx6, which is the weight variable to be used for survey participants who had not only in-person interview data but also examination and laboratory data. ${ }^{125}$ 
Because we analyzed pooled NHANES data across years 1999 to 2004, we created a 6-year sample weight variable for these analyses. In addition, we also created a 4-year sample weight for analyses involving data from survey years 2001-2004. These variables had to be created since NHANES data files only contain 2-year sample weights and a 4 year sample weight for NHANES 1999-2004. The 6-year (1999-2004) sample weight variable was created as follows: 1) the 4-year sample weight variable created by NCHS staff for participants sampled from 1999-2002 (labeled by NCHS as MEC4YR) was downloaded, 2) the 2-year sample weight created by NCHS staff for participants sampled from 2003-2004 (labeled by NCHS as MEC2YR) was downloaded, 3) a new variable was created based on the multiplication of the MEC4YR variable by $2 / 3$ if the participant was sampled from the 1999-2000 or the 2001-2002 survey years data file and multiplication by $1 / 3$ if the participant was sampled from the 2003-2004 survey years data file. The multiplication by 2/3 was done for participants sampled from 1999-2002 because they are contributing 2/3 of the total 6 years of weights while the multiplication by $1 / 3$ was done for participants sampled from 2003-2004 because they are contributing only $1 / 3$ of the total 6 years of weights. The 2001-2004 weight variable was created by dividing the weight from the 2001-2002 or the 2003-2004 by half, depending on which of the two 2-year survey cycles the person was sampled in.

\section{Primary sampling units (PSU) and strata variables:}

The NHANES uses various methods and survey software to calculate sampling errors, which is used to determine the reliability of the statistics performed. These variables, however, are not disclosed to the public. Variances thus need to be calculated 
based on masked variance units (MVUs). Variance units are based on strata and PSU variables. The NCHS created these PSU and strata variables and has them available in all NHANES data files. These variables are labeled sdmvpsu and sdmvstra for the NHANES 1999 to 2004 cycles, and sdppsu6 and sdpstra6 for NHANES III. Both PSU and strata variables are used to provide appropriate adjustment in all statistical models for the complex sample design of the NHANES.

\section{Data management and preparation}

The public-use NHANES data files were downloaded from the NCHS website (http://www.cdc.gov/nchs/nhanes.htm). The consistency of variable labels across all cycle years was checked; variable labels were changed if needed. Data files were then appended. Certain variables were redefined in order to allow for appendage. In order to determine the extent of missing data, the presence of outliers (by looking at extreme values outside the upper or lower quartiles in boxplots), and any non-normality issues, frequency distributions were performed for discrete variables, and mean, standard deviation, and range for continuous variables were checked. Normality plots were also done. Transformations were not performed. Extreme outliers were not found.

\section{$\underline{\text { Missing data }}$}

In addition to variables that are naturally defined as missing because they were left blank, values that were defined as "don't know" were redefined as missing and excluded from the analyses. Following the NCHS rule for missing data, if a variable had less than $<10 \%$ missing data, that variable was used. However, if there was more missing 
data for a variable, sample characteristics were compared in terms of demographic variables (e.g. age, sex, race/ethnicity) to determine if there were any nonrandom missing data pattern that may indicate selection bias. If there were no differences in demographic characteristics, then an imputation method was proposed to be used to replace missing values. In the present analyses however, none of the variables used had more than $10 \%$ missing data (responses of "don’t know" or left blank) and therefore imputation method not used. Variables that were omitted in the analyses were those that were asked of only a small portion of survey participants. The variables omitted from the analyses included variables related to the frequency and duration of physical activity and variables related to the frequency and the number of alcohol beverages consumed.

\section{Model criteria and strategy}

The purpose or goal of the present study was to test the proposed hypotheses derived from the research questions. Because for the first research question there was a relatively small sample and in order to avoid an over-parameterized model, ${ }^{126,}{ }^{127}$ parsimonious models (i.e. models with less variables) that would best explain the relationship between the main independent (predictor) variables and the outcome variable while controlling for potential confounders were sought. In general, the more variables added to a model, the more significant the model is or the better fit of the model; at the same time, this may lead to confusion in the interpretation of the findings and to complex interactions. ${ }^{126,127} 128$ In this study, model evaluation was not based on the overall fit or model significance, but rather the inclusion of the most important variables that if were otherwise omitted could lead to a type I error (false positive). ${ }^{126,127} 128$ 
This method of model evaluation allowed for a parsimonious model with mainly important and clinically relevant variables and ease of interpretation of findings. Variables were added in a step-wise fashion (i.e. one at a time) with the main independent variable added first. At each time a variable was added, any changes in the coefficients and the p-value of the main independent variables as well as other covariates of interest in the model were noted; changes of $20-25 \%$ of the coefficients were considered meaningful. ${ }^{126}$ Thus, the assessment of the interactions and/or confounding was possible using this approach. The approach used in the present analyses is not the same as stepwise modeling. This stepwise modeling approach is mainly concerned with the inclusion of variables that are statistically significant. ${ }^{126,127}$

Variables that were candidates for inclusion in the models were those that were pre-determined based on the literature to have clinical significance and/or that were significant at the 0.20 alpha level in univariable analyses. The 0.20 alpha level was chosen in order to allow for inclusion of variables that were perhaps clinically significant but not statistically significant at a stricter 0.05 alpha level; the age group, race/ethnicity, and sex variables were automatically included in all models regardless of statistical significance. $^{126,127}$

However, since a parsimonious model was sought and because by definition a confounder has to be associated with both the main predictor variable and the outcome variable, only variables associated with both were used in the multivariable regression analyses (either logistic, multinomial, or Poisson models). For example, since occupational group was one of the potentially new factors of interest which was associated with glycemic control at the univariable level, variables associated with both 
occupational group and with glycemic control at the univariable level were included in the multivariable logistic regression model as well as were age group, sex, and race/ethnicity.

\section{$\underline{\text { Interaction terms }}$}

Several potential interactions were tested based on other studies that found them to be important. ${ }^{36,41,55,129,130}$ The following interactions were tested for each hypothesis:

Hypotheses 1a-1d:

○ 'age group'* sex

○ 'age group'*race/ethnicity

○ race/ethnicity*education

○ race/ethnicity*sex

○ physical activity*sex

○ occupation*marital status

○ desire to lose weight*sex.

Hypotheses 2a-2c:

○ 'age group'*sex

○ 'age group'*race/ethnicity

○ race/ethnicity*education

○ race/ethnicity*sex,

○ 'suboptimal glycemic control'*sex'

○ 'borderline glycemic control'* sex

○ 'poor glycemic control' ${ }^{*}$ sex. 
Hypotheses 3a-3b:

$\circ \quad$ 'age group'*sex

○ age group*race/ethnicity

○ race/ethnicity*sex

○ sex*education,

○ 'suboptimal glycemic control'*sex'

○ 'borderline glycemic control'*sex

○ 'poor glycemic control'*sex, and sex*'BMI category'

Only statistically significant interactions at the 0.05 alpha level were included in the adjusted models or discussed in the results sections for each hypothesis.

\section{$\underline{\text { Statistical analyses }}$}

All analyses were conducted using STATA 10.0 statistical software (College Station, TX, USA) because of its ability to adjust for the complex sample survey design of the NHANES. The type I error was set at 0.05 , and an observed statistic was deemed statistically significant if the association was at or below the alpha 0.05 level. Several statistical analyses were performed after the preliminary analyses. Preliminary analyses included assessing if the assumptions to perform the regression analyses were met. For example, before performing multivariable logistic regression analyses, the linearity in the residuals was assessed using residual versus predicted plots and scatter plots, for each predictor variable. The assumption of independence and randomness was checked by looking at any patterns in the residuals. Nevertheless, independence and randomness are 
more likely because this was a cross-sectional study (except for the mortality analyses) with a multi-stage complex sampling frame that includes a random selection of participants at the first stage of sampling. The proportionality assumption for Cox proportional hazard analyses was also assessed. The SVYSET and SVY commands in STATA, for indicating survey analyses, take into account the complex survey sample, and were used for all univariable and multivariable regression analyses.

In general, first descriptive statistics and other preliminary analyses such as chisquare tests were performed. ${ }^{131}$ Chi-square tests were done in order to evaluate if there were statistically significant differences in the percentage of the outcome variables based on the independent variable. In addition, cross-tabulations allowed for the assessment of any cell sizes of less than 5 that could possibly lead to unstable regression estimates or prevent regression models to run properly. Second, univariable regression analyses were performed in order to evaluate the un-adjusted relationship (e.g. odds ratio or hazards rate) between the outcome variable and each independent variable. Third, multivariable regression analyses were performed with only clinically and/or statistically significant covariates included in the final models while controlling for potential confounders. ${ }^{127}$ Dummy variables were automatically created by STATA for categorical variables when performing regression analyses. However, when testing interactions, dummy variables were created manually (e.g. a variable with 4 categories would have 3 dummy variables, each dummy variable as 0 vs. 1).

Where the literature suggested the need for stratified analyses (e.g. sex-specific analyses) or the use of variables in a different fashion (e.g. numerical versus categorical) or using different categorization, analyses in addition to the ones proposed were also 
conducted. Moreover, mediation tests, such as the Sobel test, ${ }^{132}$ were also performed to help explain the association (or indirect effect) between the main independent variable being tested and the outcome variable. The indirect effect of the mediator is the product of path " $\mathrm{a}$ " (the estimate of the independent variable predicting the mediator) and " $\mathrm{b}$ " (the estimate of the mediator predicting the dependent variable). This test is conducted by first determining the standard error of the indirect effect, dividing the estimate of the path "ab" with the standard error of the indirect effect; a Z test is then used comparing this ratio with a critical value for a given alpha level. ${ }^{132}$ Statistical analyses for each hypothesis derived from the different research questions are described below.

\section{Hypothesis 1 a}

It was hypothesized that individuals with type 2 diabetes who worked more hours were more likely to have suboptimal, borderline, and/or poor glycemic control, after controlling for potential confounders.

\section{Hypothesis $1 b$}

It was hypothesized that individuals with type 2 diabetes who had occupations which are known for having stricter or demanding time schedules (i.e. service workers and blue collar workers relative to white collar workers) were more likely to have suboptimal, borderline, and/or poor glycemic control, after controlling for potential confounders. 


\section{Hypothesis 1c:}

It was hypothesized that individuals with type 2 diabetes who were exposed to secondhand smoke that were self-reported non-smokers were more likely to have suboptimal, borderline, and/or poor glycemic control compared to non-smokers not exposed to secondhand smoke, after controlling for potential confounders.

\section{Hypothesis 1d:}

It was hypothesized that individuals with type 2 diabetes who desired to lose weight were more likely to have suboptimal, borderline, and/or poor glycemic control, after controlling for potential confounders.

The NHANES 1999-2004 merged data file was used to test hypotheses 1a-1d using the sample of adults classified with type 2 diabetes. Only the employed sample, based on self-report of being employed, was used when testing the association between glycemic control and occupation and work hours. However, when using the desire to lose weight and the secondhand smoke exposure as independent variables, the full sample (i.e. regardless of employment) was used.

Chi-square tests were first conducted cross-tabulating the independent variables separately with each of the three measures of glycemic control status (i.e. suboptimal, borderline, and poor glycemic control). For the independent variables that were continuous, $\mathrm{t}$ tests were conducted instead of chi-square tests; these variables had normal distributions. After these preliminary analyses, univariable and multivariable logistic regression analyses were performed since the dependent variables were each dichotomous variables. The LOGISTIC command in STATA was used. The dependent variables were suboptimal, borderline, and poor glycemic control ( 0 if no, 1 if yes). 


\section{Hypothesis $2 a$}

It was hypothesized that individuals with type 2 diabetes who had suboptimal, borderline, and poor glycemic control would be more likely to have poor health-related quality of life (HRQOL) (i.e. poor self reported general health, more days with poor mental and physical health, and more days of inactivity in the past 30 days), after controlling for potential confounders.

The NHANES 2001-2004 data were used to test hypothesis 2a, using the sample of adults classified with type 2 diabetes. Chi-square tests were first conducted crosstabulating separately the four HRQOL measures with the three variables for glycemic control status. Univariable analyses for the general health variable were performed since it was a dichotomous variable. Univariable and multivariable multinomial (polychotomous) logistic regressions were then performed for dependent variables for number of days of poor mental health, poor physical health, and limited activity since these dependent variables are each categorical with three levels. The MLOGIT command in STATA was used, which does not assume ordering of the dependent variable categories. The dependent variables are the four HRQOL measures [1) general health, 2) days of poor mental health, 3) days of poor physical health, and 4) days of limited activity due to poor health in the past 30 days]). For the general health variable, 'excellent health, very good, and good' was the reference group, and it was compared to a response of 'fair or poor health'. For the 'days or poor mental health' and 'days of poor physical health' variables, '0 days with poor health' was the reference group, and it was compared to '1-13 days of poor health' and ' $\geq 14$ days of poor health'. Similarly, for the 
'days with limited activity' variable, '0 days with inactivity' was the reference group, and it was compared to ' $1-13$ days of inactivity' and ' $\geq 14$ days of inactivity'.

\section{Hypothesis $2 b$}

It was hypothesized that individuals with type 2 diabetes who had suboptimal, borderline, and poor glycemic control would have a greater number of missed work days in the past 12 months, after controlling for potential confounders.

The NHANES 1999-2004 data were analyzed using the sample of adults classified with type 2 diabetes. Univariable and multivariable Zero Inflated Poisson (ZIP) regression analyses were performed since the dependent variable, missed work days, was a count variable associated with rare events. The variable missed work days also showed over-dispersion (i.e., the mean was larger than the variance, with dispersion index or variance-to-mean ratio [VMR]) of 126) and excess zeros (over 50\% were zero). Zeroinflated Poisson (ZIP) regression models were used because, unlike traditional Poisson regression, this analytic approach is robust even in the presence excess zeros and overdispersions. ${ }^{133,134}$ The ZIP regression protocol was used for these analyses because STATA is capable of incorporating adjustments for the NHANES complex sample survey design. ${ }^{133,134}$

\section{Hypothesis $2 c$}

It was hypothesized that individuals with type 2 diabetes who had suboptimal, borderline, and poor glycemic control would be less likely to be employed, after controlling for confounders. 
The NHANES 1999-2004 merged data file was used to test hypothesis 2c, using all adults classified with type 2 diabetes. Chi-square tests were first conducted, crosstabulating employment status and the three glycemic control variables separately. Univariable and multivariable logistic regression analyses were then performed since the dependent variable was a dichotomous variable, employed [1) yes, 2) no (reference group)]. The LOGISTIC command in STATA was used.

\section{Hypotheses $3 a$}

It was hypothesized that individuals with type 2 diabetes who had suboptimal, borderline, and poor glycemic control would have a greater risk of all-cause mortality, after controlling for potential confounders.

The NHANES III (1988-1994) data file was used to test hypothesis 3a, using all adults classified with type 2 diabetes. Cox proportional hazards regression analyses were performed since the dependent variable was time to death (the event of interest) and the death indicator was defined as 1 if the person died, and 0 if otherwise (event-free or censored). The STCOX command in STATA was used.

\section{Hypotheses $3 b$}

It was hypothesized that individuals with type 2 diabetes who had suboptimal, borderline, and poor glycemic control would have a greater CVD mortality risk, after controlling for potential confounders.

The NHANES III (1988-1994) data file was used to test hypothesis 3a, using all

adults classified with type 2 diabetes. Cox proportional hazards regression analyses were 
performed since the dependent variable was time to death (the event of interest) and the death indicator was defined as 1 if the person died, and 0 if otherwise (event-free or censored). The STCOX command in STATA was used. 


\section{CHAPTER IV.}

\section{RESULTS}

\section{$\underline{\text { Overview }}$}

All statistical analyses for the present study are described for adults with type 2 diabetes and were conducted using weighted statistics and using STATA software 10.0. Table 1 provides a summary of the STATA commands used in this study. Descriptive statistics for all NHANES datasets used are shown first. For ease of presentation (e.g. to avoid very long tables) descriptive tables and tables of preliminary analyses (e.g. crosstabulations) are generally divided into the following sections: 1) demographics, 2) potentially new risk factors, and 3) health indicators and behaviors. Following these tables, the main results (i.e., multivariable regression models) are presented based on the research question and hypothesis tested. Results are divided into sections based on the outcome variable. For example, for hypotheses $1 \mathrm{a}-1 \mathrm{~d}, 2 \mathrm{a}$, and $3 \mathrm{a}-3 \mathrm{~b}$, the results are shown based on the glycemic control variable used (i.e. suboptimal, borderline, or poor glycemic control). For research question $2 \mathrm{a}$, the results are shown based on the variable for HRQOL used (i.e. self-reported general health, poor mental or physical health, and days of inactivity). The results for each hypothesis are embedded in the section for that hypothesis. A summary of results for each research question is also included at the end of the results for that research question. 


\begin{tabular}{|c|c|}
\hline \multicolumn{2}{|c|}{ Table 1. List of key STATA commands used in the present study } \\
\hline STATA Command & Purpose \\
\hline svy & Indicates analyses of surveys, weighted analyses \\
\hline svyset & $\begin{array}{l}\text { Sets all future STATA commands to be using weighted } \\
\text { analyses of survey data }\end{array}$ \\
\hline svy: tab & For cross-tabulations of weighted analyses of survey data \\
\hline stset & $\begin{array}{l}\text { Sets all future STATA commands to be using weighted } \\
\text { mortality analyses of survey data. For example the following } \\
\text { commands were written in STATA: } \\
\text { svyset sdmvpsu [pweight=mec6yr], strata (sdmvstra) }\end{array}$ \\
\hline svy: mean & For means of weighted analyses of survey data \\
\hline svy: reg & $\begin{array}{l}\text { For univariable and multivariable regression analyses of } \\
\text { weighted analyses of survey data }\end{array}$ \\
\hline svy: logistic & $\begin{array}{l}\text { For univariable and multivariable logistic regression analyses } \\
\text { of weighted analyses of survey data }\end{array}$ \\
\hline st cox & $\begin{array}{l}\text { For Cox-proportional analyses of weighted analyses of } \\
\text { survey data }\end{array}$ \\
\hline svy: mlogit & $\begin{array}{l}\text { For multinomial logistic regression analyses of weighted } \\
\text { analyses of survey data }\end{array}$ \\
\hline svy: zip & $\begin{array}{l}\text { For Zero Inflated Poisson (ZIP) regression analyses of } \\
\text { weighted analyses of survey data }\end{array}$ \\
\hline pweight & $\begin{array}{l}\text { Indicates that a weight will be used in the analyses, precedes } \\
\text { the weight variable used }\end{array}$ \\
\hline sdmvpsu & Indicates that a psu variable is being used in the analyses \\
\hline strata & $\begin{array}{l}\text { Indicates that a strata variable will be used in the analyses, } \\
\text { precedes the strata variable used }\end{array}$ \\
\hline xi: & $\begin{array}{l}\text { Indicates that categorical variables will be used in the } \\
\text { analyses and tells STATA to automatically create dummy } \\
\text { variables for the categorical variables. For example the } \\
\text { following commands were written in STATA: } \\
\text { xi: svy, subpop (if adultdiabetic): logistic suboptimalalc } \\
\text { i.workhours }\end{array}$ \\
\hline i. & $\begin{array}{l}\text { Must precede the categorical independent variable being } \\
\text { used and indicates that the variable should be made into } \\
\text { dummy variables automatically (i.e. not manually) by } \\
\text { STATA software }\end{array}$ \\
\hline subpop & $\begin{array}{l}\text { Indicates that the analyses will only be using a subsample of } \\
\text { the entire date set file and usually follows the "svy" } \\
\text { command }\end{array}$ \\
\hline
\end{tabular}




\section{General characteristics of study sample}

The general characteristics of the study sample for all NHANES data files are described in Tables 2a-2d. A total of 1273 individuals classified as type 2 diabetics aged 20 years or older who participated in the NHANES surveys anytime from 1999-2004 were included in the study. Of these, 384 (28.5\%) were employed (at a job or business in the prior week). In the 2001-2004 NHANES survey period, there were a total of 827 individuals classified as type 2 diabetes, aged 20 years or older, while there were a total of 1381 individuals with type 2 diabetes, aged 20 years and older, who participated in NHANES III (1988-1994) and who had mortality data available. There was a fairly equal representation of males and females across all NHANES.

The mean age across all NHANES data files ranged from 53 (in the employed NHANES 1999-2004 sample) to 65 (in the NHANES III sample). The mean duration of diabetes ranged from 8.7 years (employed NHANES 1999-2004 sample) to 13.2 years (NHANES 2001-2004 sample). Furthermore, over half of the study sample had at least a high school education irrespective of survey period. The sample had a slightly greater percentage of individuals who were married or living with a partner versus single, divorced or widowed across all survey periods. The majority of the sample across all NHANES survey periods was overweight or obese (79.3 to $85.2 \%)$. In the NHANES 1999-2004, among all adults with type 2 diabetes, there were $671(52.7 \%)$ with suboptimal glycemic control (HbA1c $\geq 7), 449$ (42.7\%) with borderline glycemic control (i.e. $7 \geq \mathrm{HbA} 1 \mathrm{c}<9)$, and $222(17.4 \%)$ with poor glycemic control $(\mathrm{HbA} 1 \mathrm{c} \geq 9)$. In the employed NHANES 1999-2004 sample, there were 216 (58.5\%) adults with suboptimal glycemic control, $134(46.7 \%)$ with borderline glycemic control, and $82(22.2 \%)$ with 
poor glycemic control. In the NHANES 2001-2004, there were 423 (48.0\%) adults with suboptimal glycemic control, 289 (38.7\%) with borderline glycemic control, and 134 $(15.2 \%)$ had poor glycemic control. In the NHANES III data file, there were 507 (43.2\%) adults with suboptimal glycemic control, 204 (23.4\%) with borderline glycemic control, and $303(25.8 \%)$ with poor glycemic control. There were a total of $600(43.5 \%)$ deaths from 1986 to 2000 among adults with type 2 diabetes sampled in NHANES from 19862000. Of these deaths, 315 (52.5\%) were due to cardiovascular disease.

Table 2a. Demographic characteristics among individuals classified with type 2 diabetes, 20+ years of age, NHANES III (1988-1994) and NHANES 1999-2004

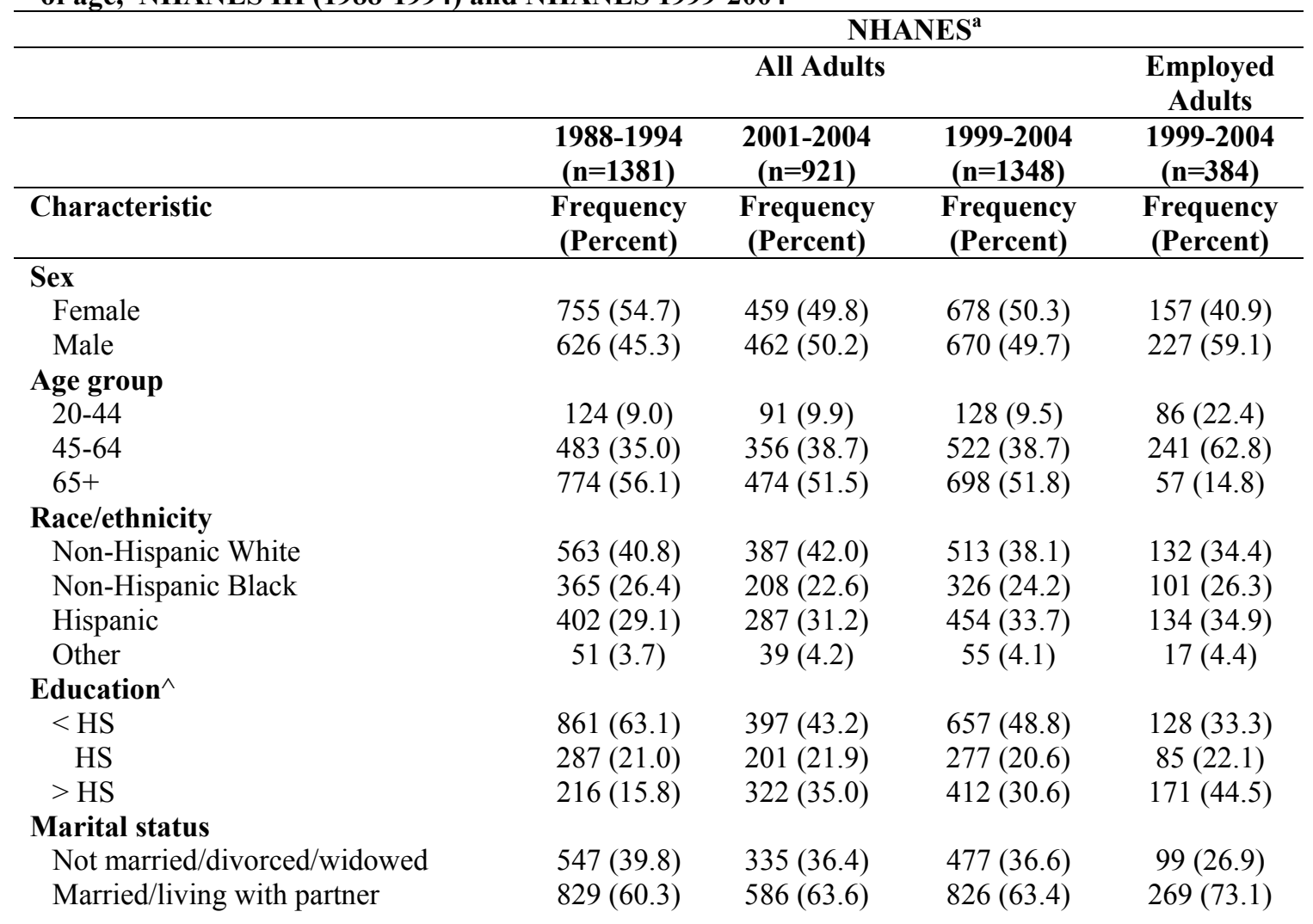


Table 2a (continue)

\begin{tabular}{lcccc}
\hline & All Adults & & $\begin{array}{c}\text { Employed } \\
\text { Adults }\end{array}$ \\
\hline & $\begin{array}{c}\mathbf{1 9 8 8 - 1 9 9 4} \\
\mathbf{( n = 1 3 8 1 )}\end{array}$ & $\begin{array}{c}\mathbf{2 0 0 1 - 2 0 0 4} \\
\text { (n=921) }\end{array}$ & $\begin{array}{c}\mathbf{1 9 9 9 - 2 0 0 4} \\
\text { (n=1348) }\end{array}$ & $\begin{array}{c}\mathbf{1 9 9 9 - 2 0 0 4} \\
\text { (n=384) }\end{array}$ \\
\hline & $\begin{array}{c}\text { Frequency } \\
\text { (Percent) }\end{array}$ & $\begin{array}{c}\text { Frequency } \\
\text { (Percent) }\end{array}$ & $\begin{array}{c}\text { Frequency } \\
\text { (Percent) }\end{array}$ & $\begin{array}{c}\text { Frequency } \\
\text { (Percent) }\end{array}$ \\
\hline $\begin{array}{c}\text { Employment status } \\
\text { Unemployed }\end{array}$ & - & & & \\
$\begin{array}{c}\text { Employed } \\
\text { Insurance status } \\
\text { Uninsured } \\
\text { Insured }\end{array}$ & -- & $2656(71.3)$ & $963(71.5)$ & --- \\
\hline
\end{tabular}

${ }^{a}$ Sample sizes do not always equal column totals due to missing data.

$\wedge$ HS=High School

Table 2b. Presence of potentially new risk factors among individuals classified with type 2 diabetes, 20+ years of age, NHANES III (1988-1994) and NHANES 1999-2004

\begin{tabular}{|c|c|c|c|c|}
\hline & \multicolumn{4}{|c|}{ NHANES $^{a}$} \\
\hline & \multicolumn{3}{|c|}{ All Adults } & \multirow{2}{*}{$\begin{array}{c}\text { Employed } \\
\text { Adults } \\
1999-2004 \\
(n=384)\end{array}$} \\
\hline & $\begin{array}{c}1988-1994 \\
(n=1381)\end{array}$ & $\begin{array}{c}2001-2004 \\
(n=921)\end{array}$ & $\begin{array}{c}1999-2004 \\
(n=1348)\end{array}$ & \\
\hline Characteristic & $\begin{array}{l}\text { Frequency } \\
\text { (Percent) }\end{array}$ & $\begin{array}{l}\text { Frequency } \\
\text { (Percent) }\end{array}$ & $\begin{array}{l}\text { Frequency } \\
\text { (Percent) }\end{array}$ & $\begin{array}{l}\text { Frequency } \\
\text { (Percent) }\end{array}$ \\
\hline \multicolumn{5}{|l|}{$\begin{array}{l}\text { Smoking and secondhand smoke } \\
\text { (SHS) exposure }\end{array}$} \\
\hline Non-Smoker \& no SHS exposure & $185(15.8)$ & $187(21.4)$ & $357(28.5)$ & $77(21.0)$ \\
\hline Non-Smoker with SHS exposure & $685(58.3)$ & $485(55.5)$ & $629(50.2)$ & $195(53.3)$ \\
\hline Smoker & $306(26.0)$ & $202(23.1)$ & $267(21.3)$ & $94(25.7)$ \\
\hline \multicolumn{5}{|l|}{ Weight desirability } \\
\hline Stay the same or gain weight & --- & $326(35.4)$ & $498(37.0)$ & $113(29.4)$ \\
\hline Lose weight & --- & $595(64.6)$ & $849(63.0)$ & $271(70.6)$ \\
\hline \multicolumn{5}{|l|}{ Hours worked } \\
\hline $0-20$ hours & --- & --- & --- & $54(14.8)$ \\
\hline 21-40 hours & --- & --- & --- & $188(51.5)$ \\
\hline $41+$ hours & --- & --- & --- & $123(33.7)$ \\
\hline \multicolumn{5}{|l|}{ Occupational group } \\
\hline White collar worker & --- & --- & --- & $177(46.2)$ \\
\hline Service worker & --- & --- & --- & $78(20.3)$ \\
\hline Farm worker & --- & --- & --- & $10(2.6)$ \\
\hline Blue collar worker & --- & --- & --- & $118(30.8)$ \\
\hline
\end{tabular}

${ }^{a}$ Sample sizes do not always equal column totals due to missing data. 
Table 2c. Presence of glycemic control group characteristics among individuals classified with type 2 diabetes, 20+ years of age, NHANES III (1988-1994) and NHANES 1999-2004

\begin{tabular}{|c|c|c|c|c|}
\hline & \multicolumn{4}{|c|}{ NHANES $^{\mathbf{a}}$} \\
\hline & \multicolumn{3}{|c|}{ All Adults } & \multirow{2}{*}{$\begin{array}{c}\text { Employed } \\
\text { Adults }\end{array}$} \\
\hline & $\begin{array}{c}1988-1994 \\
(n=1381)\end{array}$ & $\begin{array}{c}2001-2004 \\
(n=921)\end{array}$ & $\begin{array}{c}1999-2004 \\
(n=1348)\end{array}$ & \\
\hline Characteristic & $\begin{array}{c}\text { Frequency } \\
\text { (Percent) }\end{array}$ & $\begin{array}{l}\text { Frequency } \\
\text { (Percent) }\end{array}$ & $\begin{array}{c}\text { Frequency } \\
\text { (Percent) }\end{array}$ & $\begin{array}{c}\text { Frequency } \\
\text { (Percent) }\end{array}$ \\
\hline \multicolumn{5}{|c|}{$\begin{array}{l}\text { Suboptimal glycemic } \\
\text { control }^{\mathbf{b}}\end{array}$} \\
\hline No & $666(56.8)$ & $458(52.0)$ & $602(47.3)$ & $153(41.5)$ \\
\hline Yes & $507(43.2)$ & $423(48.0)$ & $671(52.7)$ & $216(58.5)$ \\
\hline \multicolumn{5}{|c|}{ Borderline glycemic control $^{\mathrm{c}}$} \\
\hline No & $666(76.6)$ & $458(61.3)$ & $602(57.3)$ & $153(53.3)$ \\
\hline Yes & $204(23.4)$ & $289(38.7)$ & $449(42.7)$ & $134(46.7)$ \\
\hline \multicolumn{5}{|c|}{ Poor glycemic control $^{\mathrm{d}}$} \\
\hline No & $870(74.2)$ & $747(84.8)$ & $1051(82.6)$ & $287(77.8)$ \\
\hline Yes & $303(25.8)$ & $134(15.2)$ & $222(17.4)$ & $82(22.2)$ \\
\hline
\end{tabular}

${ }^{a}$ Sample sizes do not always equal column totals due to missing data.

${ }^{\mathrm{b}} \mathrm{An}$ individual is said to have suboptimal glycemic control if she or he has an HbAlc of $\geq 7.0 \%$.

${ }^{\mathrm{c}}$ An individual is said to have suboptimal glycemic control if she or he has an HbAlc 7-8.9\%. In these analyses, individuals with an $\mathrm{HbA} 1 \mathrm{c} \geq 9.0 \%$ are excluded from the analyses.

${ }^{\mathrm{d}} \mathrm{An}$ individual is said to have suboptimal glycemic control if she or he has an $\mathrm{HbA1c} \geq 9.0 \%$.

Table 2d. Categorical health indicators and behavioral characteristics among individuals classified with type 2 diabetes, 20+ years of age, NHANES III (1988-1994) and NHANES 19992004

\begin{tabular}{|c|c|c|c|c|}
\hline & \multicolumn{4}{|c|}{ NHANES $^{\mathbf{a}}$} \\
\hline & \multicolumn{3}{|c|}{ All Adults } & \multirow{2}{*}{$\begin{array}{c}\text { Employed } \\
\text { Adults } \\
1999-2004 \\
(n=384) \\
\end{array}$} \\
\hline & $\begin{array}{c}1988-1994 \\
(n=1381)\end{array}$ & $\begin{array}{c}2001-2004 \\
(n=921)\end{array}$ & $\begin{array}{c}1999-2004 \\
(n=1348)\end{array}$ & \\
\hline Characteristic & $\begin{array}{c}\text { Frequency } \\
\text { (Percent) }\end{array}$ & $\begin{array}{l}\text { Frequency } \\
\text { (Percent) }\end{array}$ & $\begin{array}{c}\text { Frequency } \\
\text { (Percent) }\end{array}$ & $\begin{array}{c}\text { Frequency } \\
\text { (Percent) }\end{array}$ \\
\hline \multicolumn{5}{|c|}{$\begin{array}{l}\text { History of cardiovascular } \\
\text { disease }\end{array}$} \\
\hline No & --- & 662 (71.9) & $937(69.5)$ & $338(88.0)$ \\
\hline Yes & --- & $259(28.1)$ & $411(30.5)$ & $46(12.0)$ \\
\hline \multicolumn{5}{|c|}{ Cancer diagnosis } \\
\hline No & $1225(88.7)$ & --- & --- & --- \\
\hline Yes & $156(11.3)$ & --- & --- & - \\
\hline \multicolumn{5}{|l|}{ Alcohol Use } \\
\hline No & -- & $370(42.8)$ & $136(36.7)$ & $555(44.0)$ \\
\hline Yes & -- & $494(57.2)$ & $235(63.3)$ & $707(56.0)$ \\
\hline \multicolumn{5}{|l|}{ Physical activity } \\
\hline None & --- & $418(51.8)$ & $634(54.3)$ & $170(45.2)$ \\
\hline Moderate & --- & $270(33.5)$ & $355(30.4)$ & $115(30.6)$ \\
\hline Vigorous & --- & $119(14.8)$ & $178(15.3)$ & $91(24.2)$ \\
\hline \multicolumn{5}{|c|}{ Take anti-diabetic Pills } \\
\hline No & $690(50.2)$ & $254(27.7)$ & 375 (27.9) & $114(29.7)$ \\
\hline Yes & $685(49.8)$ & $664(72.3)$ & $969(72.1)$ & $270(70.3)$ \\
\hline
\end{tabular}


Table 2d (continue)

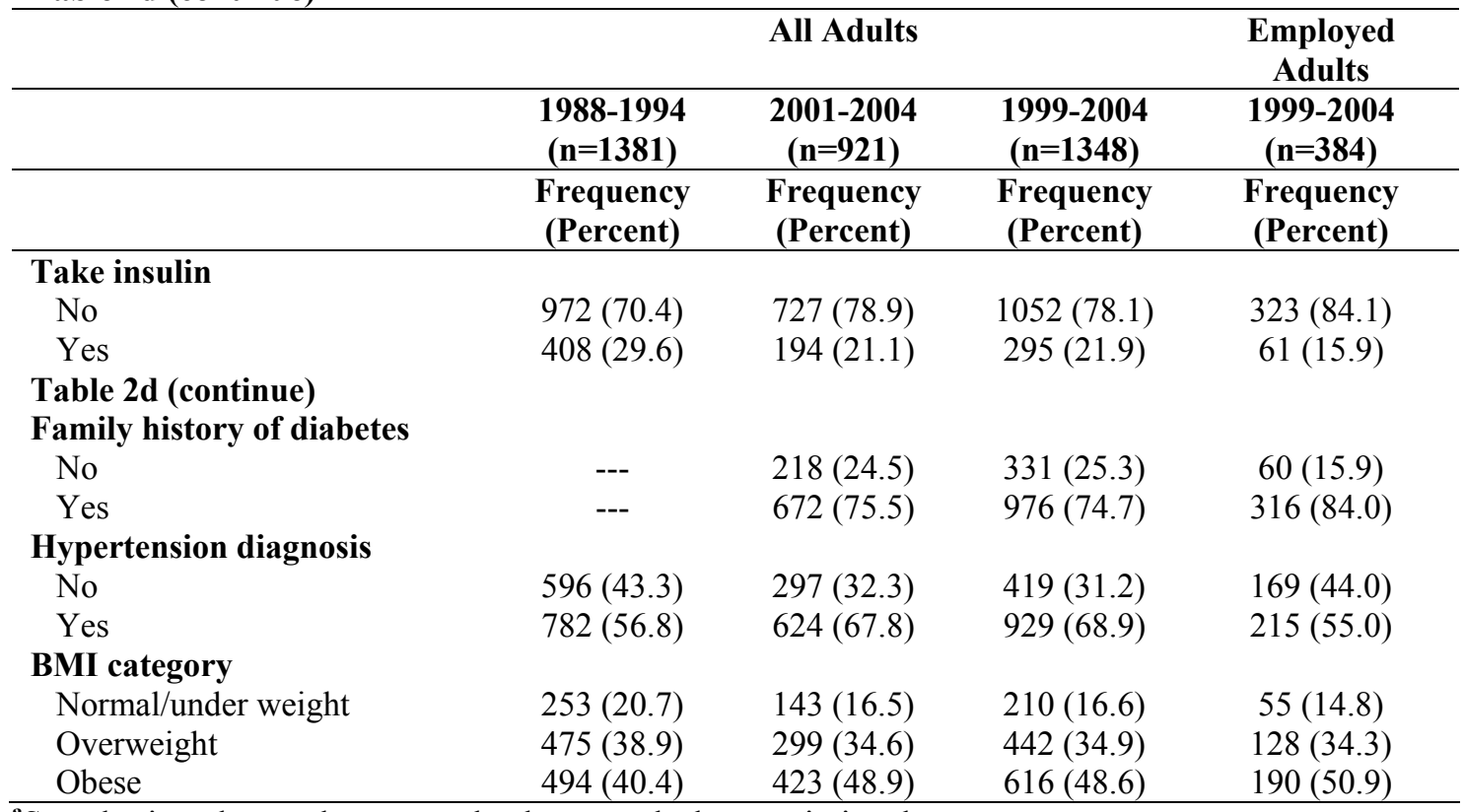

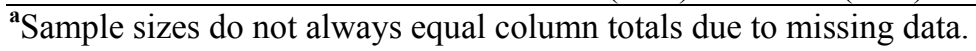

Table 2e. Numerical health indicators and behavioral characteristics among individuals classified with type 2 diabetes, 20+ years of age, NHANES III (1988-1994) and NHANES 19992004

\begin{tabular}{|c|c|c|c|c|}
\hline & \multicolumn{4}{|c|}{ NHANES $^{\mathbf{a}}$} \\
\hline & & All Adults & & Employed \\
\hline & $\begin{array}{c}1988-1994 \\
(n=1381)\end{array}$ & $\begin{array}{c}2001-2004 \\
(n=921)\end{array}$ & $\begin{array}{c}1999-2004 \\
(n=1348)\end{array}$ & $\begin{array}{c}1999-2004 \\
(n=384)\end{array}$ \\
\hline Characteristic & Mean (SE) & Mean (SE) & Mean (SE) & Mean (SE) \\
\hline Diabetes duration (years) & $10.61(9.6)$ & $13.19(15.0)$ & $12.79(14.3)$ & $8.57(10.4)$ \\
\hline Total cholesterol (mg/dl) & --- & $\begin{array}{l}199.77 \\
(47.8)\end{array}$ & $\begin{array}{l}200.27 \\
(47.1)\end{array}$ & $201.64(44.6)$ \\
\hline Albumin/Creatinine Ratio & --- & $20.34(95.9)$ & $\begin{array}{c}25.17 \\
(110.2)\end{array}$ & $21.23(136.9)$ \\
\hline $\begin{array}{l}\text { Percent calories from } \\
\text { carbohydrates }\end{array}$ & --- & $49.44(12.1)$ & $\begin{array}{l}49.51 \\
(12.2)\end{array}$ & $47.73(12.2)$ \\
\hline
\end{tabular}

${ }^{a}$ Sample sizes do not always equal column totals due to missing data. 


\section{$\underline{\text { Potentially new risk factors and glycemic control }}$}

The first research question was whether the work hours, the type of occupation, secondhand smoke exposure, and the desire to lose weight were associated with suboptimal, borderline, and poor glycemic control among individuals with type 2 diabetes who were 20 years of age or older. It was hypothesized that individuals who worked greater number of hours (i.e. $20-40$ hours or more than 40 hours relative to less than 20 hours), had occupations which are known for having stringent or demanding time schedules (i.e. service worker and blue collar workers relative to white collar workers), were exposed to secondhand smoke, and desired to lose weight were more likely to have suboptimal and/or poor glycemic control, after for potential confounders.

The associations between categorical independent variables involved in hypothesis 1a-1d and suboptimal, borderline poor, and poor glycemic control among the employed sample of adults with type 2 diabetes are tabulated and the significance of the chi-square statistics are presented in Tables 3a-3c. In addition, the means of numerical independent variables involved in hypothesis 1a-1d and significance of the t-test statistics are presented in Table 3d. Furthermore, univariable logistic regression analyses for suboptimal, borderline, and poor glycemic control among the employed sample of adults with type 2 diabetes are presented in Table $4 a-4 c$. Odds ratios are presented along with $95 \%$ confidence intervals and the corresponding $\mathrm{p}$ values. $\mathrm{P}$ values are shown only for tables $4 a-4 d$ since $p$ values at the 0.20 alpha level in the univariable analyses were used to determine which variables were to be included in subsequent multivariable analyses. 
Table 3a. Cross-tabulations ${ }^{\dagger}$ of demographic variables with suboptimal (HbA1c $\left.\geq 7.0 \%\right)$, borderline (HbA1c $\geq 7.0-8.9 \%)$, and poor $(\mathrm{HbA1c} \geq 9.0 \%$ ) glycemic control, employed adults classified with type $\overline{2}$ diabetes, NHANES 1999-2004

\begin{tabular}{|c|c|c|c|c|c|c|}
\hline & \multicolumn{6}{|c|}{ Glycemic Control* } \\
\hline & \multicolumn{2}{|c|}{ Suboptimal** } & \multicolumn{2}{|c|}{ Borderline** } & \multicolumn{2}{|c|}{ Poor** } \\
\hline & \multicolumn{2}{|c|}{$\mathbf{N}(\%)$} & \multicolumn{2}{|c|}{ N (\%) } & \multicolumn{2}{|c|}{$\mathbf{N}(\%)$} \\
\hline & No & Yes & No & Yes & No & Yes \\
\hline \multicolumn{7}{|l|}{ Sex } \\
\hline Male & $93(46.2)$ & $131(53.8)$ & $93(58.1)$ & 77 (41.9) & $170(77.0)$ & $54(23.0)$ \\
\hline Female & $60(49.5)$ & $85(50.5)$ & $60(60.0)$ & $57(40.0)$ & $117(85.3)$ & $28(14.7)$ \\
\hline \multicolumn{7}{|l|}{ Age group } \\
\hline $20-44$ & $29(42.6)$ & $51(57.4)$ & $29(58.8)$ & $27(41.2)$ & $56(72.4)$ & $24(27.6)$ \\
\hline $45-64$ & $93(47.7)$ & $140(52.3)$ & $93(59.0)$ & $85(41.0)$ & $178(80.7)$ & $55(19.3)$ \\
\hline $65+$ & $31(58.5)$ & $25(41.5)$ & $31(60.7)$ & $22(39.3)$ & $53(96.4)$ & $3(3.6)$ \\
\hline \multicolumn{7}{|l|}{ Race/ethnicity } \\
\hline Non-Hispanic & $72(55.7)$ & $56(44.3)$ & $72(65.3)$ & $39(34.7)$ & 111(85.3) & 17 (14.7) \\
\hline \multicolumn{7}{|l|}{ White } \\
\hline Non-Hispanic & $29(30.7)$ & $63(69.3)$ & $29(44.2)$ & $36(55.9)$ & $65(69.5)$ & $27(30.5)$ \\
\hline \multicolumn{7}{|l|}{ Black } \\
\hline Hispanic & $46(40.1)$ & $87(59.9)$ & $46(57.6)$ & $52(42.4)$ & $98(69.6)$ & $35(30.4)$ \\
\hline Other & $6(30.0)$ & $10(70.0)$ & $6(36.6)$ & 7 (63.6) & $13(82.2)$ & $3(17.8)$ \\
\hline \multicolumn{7}{|l|}{ Education $\wedge$} \\
\hline$<\mathrm{HS}$ & $39(33.5)$ & $86(66.5)$ & $39(46.6)$ & $49(53.4)$ & $88(72.0)$ & $37(28.0)$ \\
\hline HS & $40(51.5)$ & $40(48.5)$ & $40(65.0)$ & $26(35.0)$ & $66(79.3)$ & $14(20.7)$ \\
\hline$>\mathrm{HS}$ & $74(50.9)$ & $90(49.1)$ & $74(60.8)$ & $59(39.2)$ & 133(83.7) & $31(16.3)$ \\
\hline \multicolumn{7}{|l|}{ Marital status } \\
\hline Not married & $109(47.7)$ & $152(52.3)$ & $109(57.8)$ & $94(42.2)$ & $203(82.5)$ & $58(17.5)$ \\
\hline $\begin{array}{l}\text { Married/living } \\
\text { with partner }\end{array}$ & $38(45.8)$ & $54(54.2)$ & $38(61.7)$ & $34(38.3)$ & $72(74.3)$ & $20(25.7)$ \\
\hline \multicolumn{7}{|l|}{ Insurance Status } \\
\hline Uninsured & $32(46.1)$ & $40(53.9)$ & $32(76.6)$ & $120(57.8)$ & $48(60.1)$ & $24(39.9)$ \\
\hline Insured & $120(48.2)$ & $172(51.8)$ & $16(23.4)$ & $115(42.2)$ & $235(83.3)$ & $57(16.7)$ \\
\hline
\end{tabular}

Table 3b. Cross-tabulations ${ }^{\dagger}$ of potentially new risk factors variables with suboptimal (HbA1c $\geq$ $7.0 \%$ ), borderline (HbA1c $\geq 7.0-8.9 \%)$, and poor $(\mathrm{HbA1c} \geq 9.0 \%)$ glycemic control, Employed adults classified with type 2 diabetes, NHANES 1999-2004

\begin{tabular}{|c|c|c|c|c|c|c|}
\hline & \multicolumn{6}{|c|}{ Glycemic Control* } \\
\hline & \multicolumn{2}{|c|}{ Suboptimal** } & \multicolumn{2}{|c|}{ Borderline ${ }^{* *}$} & \multicolumn{2}{|c|}{ Poor*** } \\
\hline & \multicolumn{2}{|c|}{$\mathbf{N}(\%)$} & \multicolumn{2}{|c|}{$\mathbf{N}(\%)$} & \multicolumn{2}{|c|}{$\mathbf{N}(\%)$} \\
\hline & No & Yes & No & Yes & No & Yes \\
\hline $\begin{array}{l}\text { Smoking and } \\
\text { Secondhand } \\
\text { smoke (SHS) } \\
\text { exposure }\end{array}$ & & & & & & \\
\hline $\begin{array}{l}\text { None smoker/\& } \\
\text { no SHS }\end{array}$ & $30(48.4)$ & $47(51.6)$ & $30(57.0)$ & $31(43.0)$ & $61(88.0)$ & $16(15.0)$ \\
\hline $\begin{array}{l}\text { Non-smoker } \\
\text { with SHS }\end{array}$ & $81(47.3)$ & $111(52.7)$ & $81(60.0)$ & $67(40.0)$ & 148 (78.9) & $44(21.1)$ \\
\hline
\end{tabular}


Table 3b (continue)

\begin{tabular}{|c|c|c|c|c|c|c|}
\hline & \multicolumn{6}{|c|}{ Glycemic Control* } \\
\hline & \multirow{2}{*}{\multicolumn{2}{|c|}{$\begin{array}{c}\text { Suboptimal** } \\
\text { N (\%) }\end{array}$}} & \multicolumn{2}{|c|}{ Suboptimal** } & \multicolumn{2}{|c|}{ Suboptimal** } \\
\hline & & & & & & \\
\hline & No & Yes & No & Yes & No & Yes \\
\hline Smoker & $38(45.1)$ & $53(54.9)$ & $38(57.1)$ & $34(43.0)$ & $72(79.0)$ & $19(21.0)$ \\
\hline \multicolumn{7}{|l|}{ Weight } \\
\hline \multicolumn{7}{|l|}{ Desirability } \\
\hline $\begin{array}{l}\text { Stay the same or } \\
\text { gain weight }\end{array}$ & $48(57.6)$ & $36(42.4)$ & $48(57.6)$ & $36(42.4)$ & $84(76.3)$ & $27(23.7)$ \\
\hline Lose weight & $105(59.8)$ & $98(40.2)$ & $105(59.8)$ & $98(40.3)$ & $203(81.5)$ & $55(18.5)$ \\
\hline \multicolumn{7}{|l|}{$\begin{array}{l}\text { Hours of worked } \\
\text { group }\end{array}$} \\
\hline $0-20$ hours & $26(61.50$ & $26(38.5)$ & $26(68.3)$ & $19(31.7)$ & $45(90.0)$ & $7(10.0)$ \\
\hline 21-40 hours & $74(49.8)$ & $106(50.2)$ & $74(62.4)$ & $62(37.6)$ & $136(79.7)$ & $44(20.3)$ \\
\hline $41+$ hours & $44(38.6)$ & $75(61.4)$ & $44(50.8)$ & 47 (49.2) & $91(76.0)$ & $28(24.0)$ \\
\hline \multicolumn{7}{|l|}{$\begin{array}{l}\text { Occupational } \\
\text { group }\end{array}$} \\
\hline $\begin{array}{l}\text { White collar } \\
\text { worker }\end{array}$ & $75(53.1)$ & $91(46.9)$ & $75(62.2)$ & $61(37.8)$ & $136(85.5)$ & $30(14.6)$ \\
\hline Service worker & $28(42.5)$ & $46(57.5)$ & $28(52.1)$ & $31(47.9)$ & $59(81.7)$ & $15(18.3)$ \\
\hline Farm worker & $1(4.9)$ & $9(95.1)$ & $1(12.5)$ & $6(87.5)$ & 7 (38.9) & $3(61.1)$ \\
\hline $\begin{array}{l}\text { Blue collar } \\
\text { worker }\end{array}$ & $48(41.5)$ & $70(58.6)$ & $48(58.0)$ & $36(42.0)$ & $84(71.5)$ & $34(28.5)$ \\
\hline
\end{tabular}

Table 3c. Cross-tabulations ${ }^{\dagger}$ of health indicators and behaviors variables with suboptimal (HbA1c $\geq 7.0 \%$ ), borderline (HbA1c $\geq 7.0-8.9 \%)$, and poor $(\mathrm{HbA1c} \geq 9.0 \%)$ glycemic control, employed adults classified with type 2 diabetes, NHANES 1999-2004

\begin{tabular}{|c|c|c|c|c|c|c|}
\hline & \multicolumn{6}{|c|}{ Glycemic Control* } \\
\hline & \multirow{2}{*}{\multicolumn{2}{|c|}{$\frac{\text { Suboptimal** }}{\text { N }(\%)}$}} & \multirow{2}{*}{\multicolumn{2}{|c|}{$\frac{\text { Borderline** }}{\mathbf{N}(\%)}$}} & \multirow{2}{*}{\multicolumn{2}{|c|}{$\begin{array}{l}\text { Poor** } \\
\text { N (\%) }\end{array}$}} \\
\hline & & & & & & \\
\hline & No & Yes & No & Yes & No & Yes \\
\hline \multicolumn{7}{|c|}{ History of CVD } \\
\hline No & $132(47.2)$ & $192(52.8)$ & $132(60.1)$ & 117(39.9) & $249(78.5)$ & 75 (21.5) \\
\hline Yes & $21(49.0)$ & $24(51.0)$ & $21(53.9)$ & $17(46.1)$ & $38(91.0)$ & $7(9.0)$ \\
\hline \multicolumn{7}{|l|}{ Alcohol Use } \\
\hline No & $49(42.8)$ & $78(57.3)$ & $49(50.7)$ & $51(49.3)$ & $100(84.4)$ & $27(15.6)$ \\
\hline Yes & 101(49.7) & $131(50.3)$ & 101(63.3) & $81(36.7)$ & $182(78.5)$ & $50(21.5)$ \\
\hline \multicolumn{7}{|c|}{ Physical activity } \\
\hline None & $63(44.8)$ & $101(55.2)$ & $63(59.6)$ & $61(40.4)$ & $124(75.2)$ & $40(24.8)$ \\
\hline Moderate & $46(47.4)$ & $64(52.6)$ & $46(54.6)$ & $44(45.4)$ & $90(86.8)$ & $20(13.2)$ \\
\hline Vigorous & $39(48.9)$ & $48(51.1)$ & 39 (60.7) & $29(39.3)$ & $68(80.6)$ & 19 (19.4) \\
\hline \multicolumn{7}{|c|}{$\begin{array}{l}\text { Take anti- } \\
\text { diabetic pills }\end{array}$} \\
\hline No & $62(57.9)$ & $50(42.1)$ & $62(74)$ & $91(52.6)$ & $90(78.3)$ & $22(21.7)$ \\
\hline Yes & $91(42.6)$ & $166(57.4)$ & $28(26)$ & $106(47.4)$ & $197(81.0)$ & $60(19.0)$ \\
\hline
\end{tabular}


Table 3c (continue)

\begin{tabular}{|c|c|c|c|c|c|c|}
\hline & \multicolumn{6}{|c|}{ Glycemic Control* } \\
\hline & \multirow{2}{*}{\multicolumn{2}{|c|}{$\frac{\text { Suboptimal** }}{\text { N (\%) }}$}} & \multirow{2}{*}{\multicolumn{2}{|c|}{$\begin{array}{c}\text { Suboptimal** } \\
\mathbf{N}(\%)\end{array}$}} & \multirow{2}{*}{\multicolumn{2}{|c|}{$\begin{array}{c}\text { Suboptimal** }^{* *} \\
\mathbf{N}(\%)\end{array}$}} \\
\hline & & & & & & \\
\hline & No & Yes & No & Yes & No & Yes \\
\hline \multicolumn{7}{|l|}{ Take insulin } \\
\hline No & $140(50.8)$ & 171(49.2) & $140(62.2)$ & $107(37.8)$ & 247(81.7) & $64(18.3)$ \\
\hline Yes & $13(27.5)$ & $45(72.5)$ & $13(39.0)$ & $27(61.0)$ & 40 (70.7) & $18(29.3)$ \\
\hline \multicolumn{7}{|l|}{$\begin{array}{l}\text { Family history } \\
\text { of diabetes }\end{array}$} \\
\hline No & $33(59.4)$ & $26(40.6)$ & $33(70.1)$ & $19(29.9)$ & $52(84.7)$ & $7(15.4)$ \\
\hline Yes & $116(45.4)$ & $186(54.6)$ & $116(56.4)$ & $114(43.6)$ & $230(80.4)$ & $72(19.6)$ \\
\hline \multicolumn{7}{|l|}{$\begin{array}{l}\text { Hypertension } \\
\text { diagnosis }\end{array}$} \\
\hline No & $62(45.0)$ & $101(55.0)$ & $62(55.6)$ & $62(44.4)$ & $124(80.9)$ & $39(19.1)$ \\
\hline Yes & $91(49.6)$ & $115(50.4)$ & $91(62.5)$ & $72(37.5)$ & $163(79.4)$ & $43(20.6)$ \\
\hline \multicolumn{7}{|l|}{ BMI category } \\
\hline $\begin{array}{l}\text { Normal/under } \\
\text { weight }\end{array}$ & $22(34.7)$ & $33(65.3)$ & $22(49.9)$ & $17(50.1)$ & $39(69.5)$ & $16(30.5)$ \\
\hline Overweight & $53(54.1)$ & $71(45.9)$ & 53 (71.8) & $42(28.2)$ & $95(75.4)$ & $29(24.6)$ \\
\hline Obese & $75(46.3)$ & $108(53.7)$ & $75(54.7)$ & $72(45.3)$ & $147(84.7)$ & $36(15.3)$ \\
\hline
\end{tabular}

*row percentages $=100$, percentages do not reflect sample size in each cell due to weighted analyses.

** Statistically significant differences at the 0.05 level appear in bold.

${ }^{\dagger}$ Designed based Pearson statistics performed to account for complex survey design.

Table 3d. Mean comparisons for numerical variables with suboptimal (HbA1c $\geq 7.0 \%)$, borderline (HbA1c $\geq 7.0-8.9 \%)$, and poor $(\mathrm{HbA1c} \geq 9.0 \%$ ) glycemic control, employed adults classified with type $\overline{2}$ diabetes, NHANES 1999-2004

\begin{tabular}{lcccccc}
\hline & \multicolumn{2}{c}{ Suboptimal } & \multicolumn{2}{c}{$\begin{array}{c}\text { Glycemic Control* } \\
\text { Borderline }\end{array}$} & \multicolumn{2}{c}{ Poor } \\
\hline Characteristic & \multicolumn{2}{c}{$\begin{array}{c}\text { Mean } \\
\text { (SE) }\end{array}$} & \multicolumn{2}{c}{$\begin{array}{c}\text { Mean } \\
\text { (SE) }\end{array}$} & \multicolumn{2}{c}{$\begin{array}{c}\text { Mean } \\
\text { (SE) }\end{array}$} \\
\hline & No & Yes & No & Yes & No & Yes \\
\hline Diabetes duration & 7.07 & 8.13 & 7.07 & 8.22 & 7.55 & 8.0 \\
(years) & $(1.07)$ & $(0.78)$ & $(1.07)$ & $(1.14)$ & $(0.90)$ & $(0.86)$ \\
Total cholesterol (mg/dl) & $\mathbf{1 9 7 . 0}$ & $\mathbf{2 1 1 . 0}$ & 197.2 & 198.4 & $\mathbf{1 9 7 . 6 9}$ & $\mathbf{2 3 2 . 0 1}$ \\
& $\mathbf{( 6 . 2 9 )}$ & $\mathbf{( 5 . 0 2 )}$ & $(3.01)$ & $(5.15)$ & $\mathbf{( 2 . 7 9 )}$ & $\mathbf{( 8 . 7 6 )}$ \\
Albumin/Creatinine & $\mathbf{2 . 9 8}$ & $\mathbf{1 5 . 6 0}$ & $\mathbf{2 . 9 8}$ & $\mathbf{1 3 . 9 8}$ & $\mathbf{7 . 4 2}$ & $\mathbf{1 8 . 2 1}$ \\
Ratio & $\mathbf{( 0 . 6 7 )}$ & $\mathbf{( 4 . 8 7 )}$ & $\mathbf{( 0 . 6 7 )}$ & $\mathbf{( 7 . 1 4 )}$ & $\mathbf{( 2 . 9 1 )}$ & $\mathbf{( 5 . 4 5 )}$ \\
Percent calories from & 0.47 & 0.45 & 0.47 & 0.46 & 0.47 & 0.44 \\
carbohydrates & $(0.01)$ & $(0.01)$ & $(0.01)$ & $(0.012)$ & $(0.01)$ & $(0.02)$ \\
\hline
\end{tabular}

* Statistically significant differences at the 0.05 level appear in bold. 
Table 4a. Univariable logistic regression. Relationships between demographic characteristics with suboptimal (HbA1c $\geq 7.0 \%$ ), borderline $(\mathrm{HbA1c} \geq 7.0-8.9 \%)$, and poor $(\mathrm{HbA1c} \geq 9.0 \%)$ glycemic control, employed adults classified with type $\overline{2}$ diabetes, NHANES 1999-2004

\begin{tabular}{|c|c|c|c|}
\hline & \multicolumn{3}{|c|}{ Glycemic Control } \\
\hline & Suboptimal & Borderline & Poor \\
\hline Characteristic & $\begin{array}{c}\text { Odds Ratio (95\% } \\
\text { Confidence Interval), } \\
\text { p value }\end{array}$ & $\begin{array}{c}\text { Odds Ratio ( } 95 \% \\
\text { Confidence Interval), } \\
\text { p value }\end{array}$ & $\begin{array}{c}\text { Odds Ratio (95\% } \\
\text { Confidence Interval), } \\
\text { p value }\end{array}$ \\
\hline \multicolumn{4}{|c|}{ 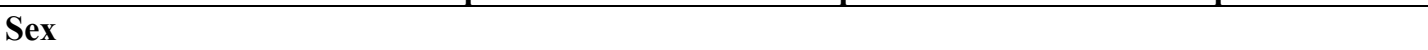 } \\
\hline Female & 1.00 & 1.00 & 1.00 \\
\hline Male & $1.14(0.60-2.17), 0.68$ & $0.92(0.49-1.74), 0.80$ & $1.73(0.87-3.44), 0.12$ \\
\hline \multicolumn{4}{|l|}{ Age group } \\
\hline $20-44$ & 1.00 & 1.00 & 1.00 \\
\hline $45-64$ & $0.81(0.38-1.76), 0.60$ & $0.99(0.41-2.37), 0.98$ & $0.63(0.26-1.50), 0.29$ \\
\hline $65+$ & $0.530(0.21-1.30), 0.16$ & $0.93(0.37-2.33), 0.87$ & $0.10(0.02-0.66), 0.02$ \\
\hline \multicolumn{4}{|l|}{ Race/ethnicity } \\
\hline Non-Hispanic & 1.00 & 1.00 & 1.00 \\
\hline \multicolumn{4}{|l|}{ White } \\
\hline Non-Hispanic & $2.84(1.56-5.17), 0.00$ & 2.37 (1.26-4.49), 0.01 & 2.55 (1.13-5.7), 0.03 \\
\hline \multicolumn{4}{|l|}{ Black } \\
\hline Hispanic & $1.88(1.11-3.12) 0.02$ & $1.38(0.87-2.20), 0.17$ & 2.54 (1.08-5.97), 0.03 \\
\hline Other & $2.94(0.74-11.64) 0.12$ & $3.28(0.73-14.72), 0.12$ & $1.26(0.39-4.06), 0.69$ \\
\hline \multicolumn{4}{|l|}{ Education $^{\wedge}$} \\
\hline$<\mathrm{HS}$ & 1.00 & 1.00 & 1.00 \\
\hline HS & $0.47(0.22-1.04), 0.06$ & $0.47(0.20-1.11), 0.08$ & $1.67(0.30-1.52), 0.33$ \\
\hline$>\mathrm{HS}$ & $0.49(0.28-0.84), 0.01$ & $0.56(0.30-1.01), 0.08$ & $0.50(0.28-0.89), 0.02$ \\
\hline \multicolumn{4}{|l|}{ Marital status } \\
\hline Not married & 1.00 & 1.00 & 1.00 \\
\hline $\begin{array}{l}\text { Married/living with } \\
\text { partner }\end{array}$ & $1.08(0.60-1.93), 0.79$ & $0.85(0.44-1.65), 0.62$ & $1.64(0.88-3.04), 0.12$ \\
\hline \multicolumn{4}{|l|}{ Insurance status } \\
\hline Uninsured & 1.00 & 1.00 & 1.00 \\
\hline Insured & $0.28(0.15-0.53), 0.00$ & $2.40(1.19-4.79), 0.02$ & $0.30(0.14-0.64), 0.00$ \\
\hline
\end{tabular}

Table $4 \mathrm{~b}$. Univariable logistic regression. Relationship between potentially new risk factors with suboptimal $(\mathrm{HbA1c} \geq 7.0 \%)$, borderline $(\mathrm{HbA1c} \geq 7.0-8.9 \%)$, and poor $(\mathrm{HbA1c} \geq 9.0 \%)$ glycemic control, employed adults classified with type 2 diabetes, NHANES 1999-2004

\begin{tabular}{lccc}
\hline & Suboptimal & Glycemic Control & Borderline \\
\hline Characteristic & $\begin{array}{c}\text { Odds Ratio (95\% } \\
\text { Confidence Interval), } \\
\text { p value }\end{array}$ & $\begin{array}{c}\text { Odds Ratio (95\% } \\
\text { Confidence Interval), } \\
\text { p value }\end{array}$ & $\begin{array}{c}\text { Odds Ratio (95\% } \\
\text { Confidence Interval), } \\
\text { p value }\end{array}$ \\
\hline $\begin{array}{l}\text { Smoking and } \\
\text { secondhand smoke } \\
\text { (SHS) exposure } \\
\quad \text { None smoker/\& no }\end{array}$ & 1.00 & & \\
SHS & & 1.00 & 1.00
\end{tabular}


Table 4b (continue)

\begin{tabular}{|c|c|c|c|}
\hline & \multicolumn{3}{|c|}{ Glycemic Control } \\
\hline & Suboptimal & Borderline & Poor \\
\hline & $\begin{array}{c}\text { Odds Ratio (95\% } \\
\text { Confidence Interval), } \\
\text { p value }\end{array}$ & $\begin{array}{c}\text { Odds Ratio (95\% } \\
\text { Confidence Interval), } \\
\text { p value }\end{array}$ & $\begin{array}{c}\text { Odds Ratio (95\% } \\
\text { Confidence Interval), } \\
\text { p value }\end{array}$ \\
\hline $\begin{array}{l}\text { Non-smoker with } \\
\text { SHS exposure }\end{array}$ & $\begin{array}{c}1.05(0.45-2.38), \\
0.92\end{array}$ & $1.72(0.27-11.01), 0.56$ & $\begin{array}{c}1.51(0.58-3.92), \\
0.39\end{array}$ \\
\hline Smoker & $\begin{array}{c}1.14(0.45-2.93), \\
0.77\end{array}$ & $0.70(0.288-1.68), 0.41$ & $\begin{array}{c}1.50(0.52-4.37) \\
0.45\end{array}$ \\
\hline \multicolumn{4}{|l|}{ Weight desirability } \\
\hline $\begin{array}{l}\text { Stay the same or } \\
\text { gain weight }\end{array}$ & 1.00 & 1.00 & 1.00 \\
\hline Lose weight & $\begin{array}{c}0.83(0.44-1.56) \\
0.55\end{array}$ & $\begin{array}{c}0.91(0.46-1.83) \\
0.80\end{array}$ & $\begin{array}{c}0.73(0.33-1.62), \\
0.43\end{array}$ \\
\hline \multicolumn{4}{|l|}{$\begin{array}{l}\text { Hours of worked } \\
\text { group }\end{array}$} \\
\hline $0-20$ hours & 1.00 & 1.00 & 1.00 \\
\hline 21-40 hours & $\begin{array}{c}1.61(0.77-3.38), \\
0.20\end{array}$ & $\begin{array}{c}1.30(0.52-3.26) \\
0.57\end{array}$ & $\begin{array}{c}2.28(0.59-8.78), \\
0.22\end{array}$ \\
\hline $41+$ hours & $\begin{array}{c}2.54(1.24-5.22) \\
0.01\end{array}$ & $\begin{array}{c}2.10(0.93-4.68), \\
0.07\end{array}$ & 2.84 (0.72-11.18), 0.13 \\
\hline \multicolumn{4}{|l|}{ Occupational group } \\
\hline White collar worker & 1.00 & 1.00 & 1.00 \\
\hline Service worker & $\begin{array}{c}1.52(0.77-3.04) \\
0.89\end{array}$ & $\begin{array}{c}1.51(0.67-3.38), \\
3.57\end{array}$ & $\begin{array}{c}1.31(0.49-3.51) \\
0.58\end{array}$ \\
\hline Farm worker & $\begin{array}{l}22.07(2.47- \\
202.11), 0.01\end{array}$ & $\begin{array}{c}11.45(1.17-112.09), \\
0.03\end{array}$ & 9.21 (1.66-51.09), 0.01 \\
\hline Blue collar worker & $\begin{array}{c}1.60(0.85-3.01) \\
0.14\end{array}$ & $\begin{array}{c}1.19(0.57-2.47), \\
0.64\end{array}$ & $\begin{array}{c}2.34(1.05-5.21), \\
0.04\end{array}$ \\
\hline
\end{tabular}

Table 4c. Univariable logistic regression. Health indicators and behaviors factors associated suboptimal (HbA1c $\geq 7.0 \%$ ), borderline $(\mathrm{HbA1c} \geq 7.0-8.9 \%)$, and poor $(\mathrm{HbA1c} \geq 9.0 \%)$ glycemic control employed adults classified with type 2 diabetes, NHANES 1999-2004

\begin{tabular}{|c|c|c|c|}
\hline & \multicolumn{3}{|c|}{ Glycemic Control } \\
\hline & Suboptimal & Borderline & Poor \\
\hline Characteristic & $\begin{array}{c}\text { Odds Ratio (95\% } \\
\text { Confidence Interval), } \\
\text { p value }\end{array}$ & $\begin{array}{c}\text { Odds Ratio (95\% } \\
\text { Confidence Interval), } \\
\text { p value }\end{array}$ & $\begin{array}{c}\text { Odds Ratio ( } 95 \% \\
\text { Confidence Interval), } \\
\text { p value }\end{array}$ \\
\hline \multicolumn{4}{|l|}{ CVD history } \\
\hline No & 1.00 & 1.00 & 1.00 \\
\hline Yes & $0.93(0.40-2.16), 0.86$ & $1.29(0.52-3.23), 0.58$ & $0.36(0.12-1.08), 0.07$ \\
\hline \multicolumn{4}{|l|}{ Alcohol use } \\
\hline No & 1.00 & 1.00 & 1.00 \\
\hline Yes & $1.35(0.96-1.90), 0.08$ & $0.60(0.32-1.17), 0.10$ & $1.48(0.82-2.66), 0.19$ \\
\hline \multicolumn{4}{|l|}{ Physical activity } \\
\hline None & 1.00 & 1.00 & 1.00 \\
\hline Moderate & $0.90(0.46-1.77), 0.76$ & $1.23(0.59-2.55), 0.58$ & $0.46(0.22-0.99), 0.05$ \\
\hline Vigorous & $0.85(0.45-1.62), 0.61$ & $0.96(0.47-1.94), 0.90$ & $0.73(0.32-1.64), 0.44$ \\
\hline
\end{tabular}


Table 4c (continue)

\begin{tabular}{|c|c|c|c|}
\hline & \multicolumn{3}{|c|}{ Glycemic Control } \\
\hline & Suboptimal & Borderline & Poor \\
\hline & $\begin{array}{c}\text { Odds Ratio (95\% } \\
\text { Confidence Interval), } \\
\text { p value }\end{array}$ & $\begin{array}{c}\text { Odds Ratio (95\% } \\
\text { Confidence Interval), } \\
\text { p value }\end{array}$ & $\begin{array}{c}\text { Odds Ratio (95\% } \\
\text { Confidence Interval), } \\
\text { p value }\end{array}$ \\
\hline \multicolumn{4}{|l|}{$\begin{array}{l}\text { Take anti-diabetic } \\
\text { pills }\end{array}$} \\
\hline No & 1.00 & 1.00 & 1.00 \\
\hline Yes & 1.17 (0.69-1.97), 0.55 & $2.57(1.39-4.72), 0.00$ & $0.85(0.39-1.81), 0.66$ \\
\hline \multicolumn{4}{|l|}{ Take insulin } \\
\hline No & 1.00 & 1.00 & 1.00 \\
\hline Yes & $2.07(1.34-3.21), 0.00$ & $2.57(1.12-5.88), 0.03$ & $1.85(0.85-4.05), 0.12$ \\
\hline \multicolumn{4}{|l|}{$\begin{array}{l}\text { Family history of } \\
\text { diabetes }\end{array}$} \\
\hline Yes & $1.30(0.78-2.14), 0.31$ & $1.61(1.13-2.27), 0.01$ & 1.34 (0.47-3.83), 0.66 \\
\hline \multicolumn{4}{|l|}{$\begin{array}{l}\text { Hypertension } \\
\text { diagnosis }\end{array}$} \\
\hline No & 1.00 & 1.00 & 1.00 \\
\hline Yes & $0.85(0.55-1.30), 0.44$ & $0.75(0.41-1.38), 0.35$ & $1.10(0.54-2.23), 0.78$ \\
\hline \multicolumn{4}{|l|}{ BMI category } \\
\hline $\begin{array}{l}\text { Normal/under } \\
\text { weight }\end{array}$ & 1.00 & 1.00 & 1.00 \\
\hline Overweight & $0.45(0.17-1.22), 0.11$ & $0.39(0.15-1.01), 0.05$ & $0.74(0.26-2.11), 0.57$ \\
\hline Obese & $0.62(0.27-1.40), 0.24$ & $0.82(0.34-1.98), 0.66$ & $0.42(0.16-1.04), 0.06$ \\
\hline $\begin{array}{l}\text { Diabetes duration } \\
\text { (years) }\end{array}$ & $0.99(0.98-1.00), 0.25$ & $1.01(0.98-1.04), 0.41$ & $1.01(0.98-1.03), 0.72$ \\
\hline $\begin{array}{l}\text { Total cholesterol } \\
\text { (mg/dl) }\end{array}$ & 1.02 (1.00-1.02), 0.00 & $1.00(0.99-1.00), 0.83$ & 1.02 (1.01-1.03), 0.00 \\
\hline $\begin{array}{l}\text { Albumin/Creatinine } \\
\text { Ratio }\end{array}$ & 1.00 (1.00-1.00), 0.05 & $1.00(1.00-1.02), 0.11$ & $1.00(0.98-1.01), 0.35$ \\
\hline $\begin{array}{l}\text { \% calories from } \\
\text { carbohydrates }\end{array}$ & $0.36(0.03-3.96), 0.40$ & $0.64(0.05-8.08), 0.73$ & 0.15 (0.01-2.53), 0.18 \\
\hline
\end{tabular}

$\underline{\text { Suboptimal glycemic control }}$

Among the employed sample, individuals with type 2 diabetes who worked greater than 40 hours relative to those who worked 20 hours or less had greater odds for suboptimal (HbA1c $\geq 7)$ glycemic control (Odds Ratio, OR, $=2.54$ [95\% confidence interval, $\mathrm{CI},=1.24-5.22])($ Tables $4 \mathrm{a}-\mathrm{c})$. In addition, farm workers were more likely than white collar workers to have suboptimal glycemic control (OR=22.07 [CI=2.40-202.11]). Other factors associated with suboptimal glycemic control at the 0.20 alpha level ( the level for determining potential confounders) included: taking insulin ( $O R=2.70[p$ 
$<0.001])$, being 65 years of age or older relative to $20-44$ years of age ( $O R=0.53$ $[\mathrm{p}=0.016)$, being insured $(0.28[\mathrm{p}<0.001])$, being non-Hispanic Black $(\mathrm{OR}=2.37$ $[\mathrm{p}=0.01])$, Hispanic (OR $1.38[\mathrm{p}=0.17])$, being of 'other' race $(\mathrm{OR}=2.94[\mathrm{p}=0.12])$, and being overweight $(\mathrm{OR}=0.39[\mathrm{p}=0.05])$. The other independent variables of interest for research question 1 (i.e. secondhand smoke exposure and desire to lose weight) were not statistically associated with suboptimal glycemic control at the 0.20 alpha level among all adults of the sample (i.e., regardless of employment status).

Multivariable model development: For the model to assess the relationship between work hours and suboptimal glycemic control, variables were entered in this logistic regression model in the following order: 1) work hours, 2) sex, 3) age group, 4) race/ethnicity, 5) occupation, 6) education, 7) insurance status, 8) taking insulin, and 9) BMI category.

These variables were added because they have shown clinical significance in the literature $^{30-39}$ and were statistically associated with work characteristics and glycemic control in the present study (see for example tables $4 a-4 d$ ).

The order for the model was based on adding variables that had the greatest magnitude in the association with glycemic control in the univariable model. Only the variables statistically associated with both work hours and glycemic control and that had clinical significance were entered in the model. There were no notable difference (i.e. change of $20 \%$ or greater in coefficients or $\mathrm{p}$ values) in the direction of the estimates (odds ratios) and the $\mathrm{p}$ values for each category of the work hours variable and the other covariates in the models at each step in the mode building procedure. 
The final multivariable logistic regression model assessing the relationship between suboptimal glycemic control, work hours, and occupation among employed adults with type 2 diabetes using NHANES 1999-2004 data are presented in Table 5. Individuals aged 20 years or older with type 2 diabetes working greater than 40 hours were more likely to have suboptimal glycemic control compared to those working 1-20 hours $(\mathrm{OR}=5.03$ [CI=1.37-18.42]), after controlling for sex, age group, race/ethnicity, occupation, education, insurance, taking insulin, and BMI category. Although not statistically significant at the 0.05 alpha level, individuals working $21-40$ hours also had greater odds for suboptimal glycemic control compared to those working 20 hours or less $(\mathrm{OR}=1.91[\mathrm{CI}=0.72-5.07])$. In addition, farm workers were more likely to have suboptimal glycemic control relative to white collar workers $(\mathrm{OR}=28.2[\mathrm{CI}=1.96-403.3])$.

\begin{tabular}{|c|c|c|}
\hline Characteristic & Odds Ratio & 95\% Confidence Interval \\
\hline \multicolumn{3}{|l|}{ Work hours } \\
\hline $1-20$ hours & 1.00 & \\
\hline 21-40 hours & 1.91 & $0.72-5.07$ \\
\hline $41+$ hours & 5.03 & 1.37-18.42 \\
\hline \multicolumn{3}{|l|}{ Sex } \\
\hline Male & 1.00 & \\
\hline Female & 0.92 & $0.35-2.44$ \\
\hline \multicolumn{3}{|l|}{ Age group (yrs) } \\
\hline $20-44$ & 1.00 & \\
\hline $45-64$ & 0.89 & $0.39-2.02$ \\
\hline $65+$ & 0.94 & $0.36-2.45$ \\
\hline \multicolumn{3}{|l|}{ Race/Ethnicity } \\
\hline Non-Hispanic White & 1.00 & \\
\hline Non-Hispanic Black & 3.36 & $1.49-7.58$ \\
\hline Hispanic & 2.10 & $0.91-4.81$ \\
\hline Other & 4.24 & $0.91-19.79$ \\
\hline \multicolumn{3}{|l|}{ Occupation } \\
\hline White collar worker & 1.00 & \\
\hline Service worker & 1.18 & $0.51-2.72$ \\
\hline Farm worker & 28.2 & $1.96-403.3$ \\
\hline
\end{tabular}




\begin{tabular}{|c|c|c|}
\hline Characteristic & Odds Ratio & 95\% Confidence Interval \\
\hline Blue Collar worker & 0.77 & $0.37-1.62$ \\
\hline \multicolumn{3}{|l|}{ Education ${ }^{\wedge}$} \\
\hline$<\mathrm{HS}$ & 1.00 & \\
\hline HS & 0.53 & $0.20-1.40$ \\
\hline$>\mathrm{HS}$ & 0.61 & $0.29-1.29$ \\
\hline \multicolumn{3}{|l|}{ Have insurance } \\
\hline No & 1.00 & \\
\hline Yes & 0.87 & $0.35-2.12$ \\
\hline \multicolumn{3}{|l|}{ Taking insulin } \\
\hline No & 1.00 & \\
\hline Yes & 3.97 & $1.45-10.89$ \\
\hline \multicolumn{3}{|l|}{ BMI category } \\
\hline Under/normal weight & 1.00 & \\
\hline Overweight & 0.48 & $0.16-1.48$ \\
\hline Obese & 0.79 & $0.34-1.82$ \\
\hline
\end{tabular}

$\wedge$ HS=High School

$\underline{\text { Borderline glycemic control }}$

Among the employed sample, univariable analyses indicated that individuals with type 2 diabetes who worked greater than 40 hours relative to those who worked 20 hours or less had greater odds for borderline glycemic control ( $\mathrm{OR}=2.09[\mathrm{CI}=0.93-4.68])$ (Table 4a-c). Although not statistically significant at the 0.20 alpha level (the level for determining potential confounders), employed individuals with type 2 diabetes who worked 21-40 hours were more likely than those who worked less than 20 hours $(\mathrm{OR}=1.30[\mathrm{CI}=0.52-3.26])$ to have borderline glycemic control. In addition, the type of occupation was marginally associated with borderline glycemic control at the 0.20 alpha level (the level for determining potential confounders). Specifically, farm workers were also more likely than white collar workers to have borderline glycemic control $(\mathrm{OR}=11.25[\mathrm{p}=0.03])$. Other factors associated with greater odds of borderline glycemic control at the 0.20 alpha level included: being a non-Hispanic Black $(\mathrm{OR}=2.37[\mathrm{p}=0.01])$, Hispanic $(\mathrm{OR}=1.38[\mathrm{p}=0.17])$, or of other race/ethnic background $(3.28[\mathrm{p}=0.12])$ relative 
to non-Hispanic whites, taking diabetic pills $(\mathrm{OR}=2.57[\mathrm{p}=<0.001])$, taking insulin $(\mathrm{OR}=2.57[\mathrm{p}=0.03])$, and having family history of diabetes $(\mathrm{OR}=1.61[\mathrm{p}=0.01])$.

The other 2 main independent variables of interest for research question 1 (i.e., secondhand smoke exposure and desire to lose weight) were not statistically associated with borderline glycemic control at the 0.20 alpha level among all adults or the employed sample.

Multivariable model development: For the model to assess the relationship between work hours, occupation, and borderline glycemic control, the order of how the variables were entered in this logistic regression model was as follows: 1) work hours category, 2) sex, 3) age group, 4), race/ethnicity, 5) occupation, 6) education, 7) insurance status, 8) taking insulin, and 9) BMI category. These variables were added because they have shown clinical significance in the literature ${ }^{30-39}$ and were statistically associated with work characteristics and glycemic control in the present study (see for example tables $4 \mathrm{a}-4 \mathrm{~d})$. The order for the model was based on adding variables that had the greatest magnitude in the association with glycemic control in the univariable model. Only the variables statistically associated with both work hours and glycemic control and that had clinical significance were entered in the model.

When sex, age group, race/ethnicity, occupation, education, and insurance status were added in the model, the direction of the estimates (odds ratios) for each category of the work hours variable and the corresponding $\mathrm{p}$ values remained relatively unchanged except that the work hours variable category of $41+$ hours became statistically significant when sex was added. When the variable 'taking insulin' was added, the odds ratio for the category for '21-40 work hours' and ' $41+$ work hours' doubled. When the BMI category 
and hypertension diagnosis were added, the direction of the estimates and the $p$ values for the work hours and the covariates (i.e., sex, age group, race/ethnicity, occupation, education, and insurance status) remained relatively unchanged.

The final multivariable logistic regression model assessing the relationship between borderline glycemic control, work hours, and occupation among employed adults with type 2 diabetes using adjusting for confounders is presented in Table 6. Individuals aged 20 years or older with type 2 diabetes working over 40 hours were more likely to have borderline glycemic control compared to those working 1-20 hours $(\mathrm{OR}=7.19[\mathrm{CI}=1.88-27.45])$, after controlling for sex, age group, race/ethnicity, occupation, education, insurance, taking insulin, and BMI category.

Table 6. Multivariable logistic regression. Relationship between borderline (HbA1c 7-8.9\%) glycemic control and work characteristics among employed adults with type 2 diabetes, NHANES 1999-2004

\begin{tabular}{lcc}
\hline Characteristic & Odds Ratio & $\mathbf{9 5 \%}$ Confidence Interval \\
\hline Work hours & 1.00 & \\
$1-20$ hours & 2.39 & $0.82-6.90$ \\
$21-40$ hours & $\mathbf{7 . 1 9}$ & $\mathbf{1 . 8 8 - 2 7 . 4 5}$ \\
$41+$ hours & & \\
Sex & 1.00 & $0.37-2.83$ \\
Male & 1.03 & \\
Female & & \\
Age group (yrs) & 1.00 & $0.43-2.57$ \\
$20-44$ & 1.04 & $0.59-6.12$ \\
$45-64$ & 1.90 & \\
65+ & & \\
Race/Ethnicity & 1.00 & $\mathbf{1 . 1 6 - 7 . 3 8}$ \\
Non-Hispanic White & $\mathbf{2 . 9 3}$ & $0.90-4.24$ \\
Non-Hispanic Black & 1.95 & $0.83-29.90$ \\
Hispanic & 4.99 & \\
Other & & \\
Occupation & 1.00 & $0.56-3.83$ \\
White collar worker & 1.47 & $0.28-1.83$ \\
Service worker & 13.30 & \\
Farm worker & 0.71 & \\
Blue Collar worker & 1.00 & \\
Education & & \\
<HS & & \\
& &
\end{tabular}




\begin{tabular}{lcc} 
Table 6 (continue) & Odds Ratio & $\mathbf{9 5 \%}$ Confidence Interval \\
\hline Characteristic & 0.36 & $0.10-1.29$ \\
\hline HS & 0.56 & $0.22-1.43$ \\
>HS & & \\
Have insurance & 2.67 & $0.98-7.27$ \\
No & & \\
Yes & 1.00 & \\
Taking insulin & $\mathbf{3 . 3 5}$ & $\mathbf{1 . 1 3 - 9 . 8 8}$ \\
No & & \\
Yes & 1.00 & \\
BMI category & 0.35 & $0.12-1.07$ \\
Under/normal weight & 1.04 & $0.38-2.81$ \\
Overweight & & \\
Obese &
\end{tabular}

${ }^{\wedge} \mathrm{HS}=$ High School

Of the interactions that were tested for the borderline glycemic control variable, only 'desire to lose weight'*race/ethnicity and 'desire to lose weight'*sex were significant. Individuals who wanted to lose weight and were of other race were significantly less likely to have borderline glycemic control compared to individuals who did not want to lose weight and were white $(\mathrm{OR}=0.23[\mathrm{CI}=0.06-0.95])$. Females who wanted to lose weight were more likely to have borderline glycemic control compared to males who did not want to lose weight $(\mathrm{OR}=2.17[\mathrm{CI}=1.18-4.02])$. The addition of these interactions in the model did not appreciably alter the OR estimates for the 'desire to lose weight' variable or the work hours or occupation variables in the logistic regression model. Therefore the interaction variable was not included in the final model shown in Table 6.

$\underline{\text { Poor glycemic control }}$

At the 0.20 alpha level, among the employed sample, individuals with type 2 diabetes who were farm workers or blue collar workers were more likely to have poor glycemic control relative to white collar workers $(\mathrm{OR}=9.21[\mathrm{CI}=1.66-51.09])$ and 
$(\mathrm{OR}=2.35[\mathrm{CI}=1.06-5.21])$, respectively (Table 2$)$. Other factors associated with greater odds of poor glycemic control included at the 0.20 alpha level included: being a nonHispanic Black $(\mathrm{OR}=2.55[\mathrm{p}=0.03])$, and Hispanic $(\mathrm{OR}=2.54[\mathrm{p}=0.03])$ relative to nonHispanic whites. Factors that were inversely related to poor glycemic control included having more than a high school education compared to having less than a high school education $(\mathrm{OR}=0.50[\mathrm{p}=0.03])$, being 65 years of age or older relative to those aged 2044 years $(\mathrm{OR}=0.10[\mathrm{p}=0.02])$, and having insurance $(0.30[\mathrm{p}<0.01)$. The other 3 main independent variables of interest for research question (i.e. work hours category, secondhand smoke exposure and desire to lose weight) were not statistically associated with poor glycemic control at the 0.20 alpha level among all adults or the employed sample.

Multivariable model development: For the model to assess the relationship between occupation and poor glycemic control, the order of how the variables were entered in this logistic regression model was as follows: 1) occupation, 2) sex, 3) age group, 4) race/ethnicity, 5) work hours category, 6) education, 7) insurance status, 8) taking insulin, and 9) BMI category.

These variables were added because they have shown clinical significance in the literature $\mathrm{e}^{30-39}$ and because they were statistically associated with work characteristics and glycemic control in the present study (see for example tables 4a-4d).

The order for the model was based on adding variables that had the greatest magnitude in the association with glycemic control in the univariable model. Only the variables statistically associated with both work hours and glycemic control and that had clinical significance were entered in the model. When age group, race/ethnicity, and work hours, 
education, insurance, taking insulin, and BMI category were added, the direction of the estimates and the $\mathrm{p}$ values for the occupation and the other covariates remained relatively unchanged.

The final multivariable logistic regression model assessing the relationship between poor glycemic control, occupation, and work hours among employed adults with type 2 diabetes using NHANES 1999-2004 data are presented in Table 7. Among individuals aged 20 years or older with type 2 diabetes and after adjusting for potential confounders such as sex, age group, race/ethnicity, work hours category, education, insurance, taking insulin, and BMI category, there was no statistically significant relationship between poor glycemic control and being a farm worker $(\mathrm{OR}=9.93[\mathrm{CI}=0.94$ 105.21]).

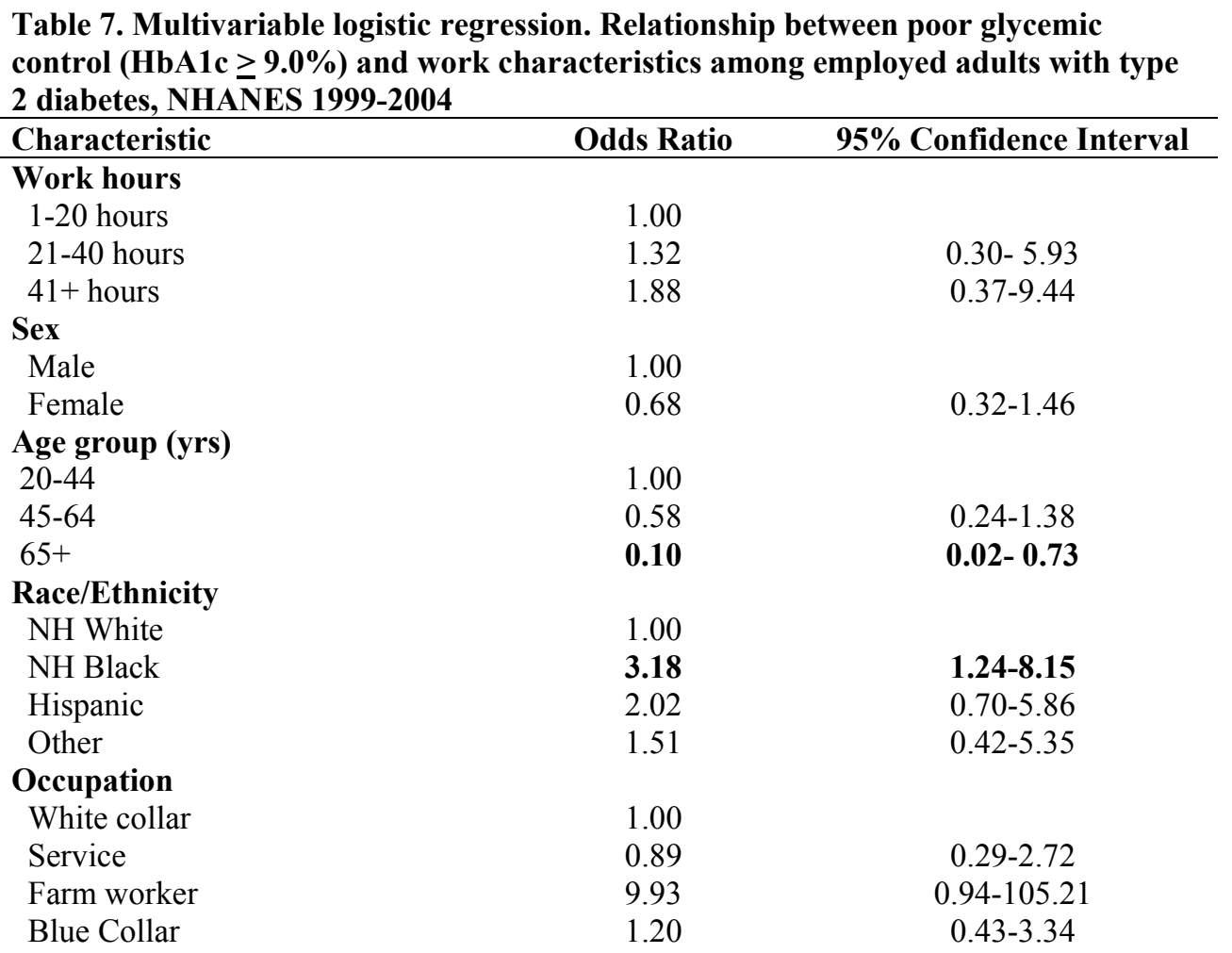


Table 7 (continue)

\begin{tabular}{lcc}
\hline Characteristic & Odds Ratio & $\mathbf{9 5 \%}$ Confidence Interval \\
\hline Education $^{\wedge}$ & & \\
$<$ HS & 1.00 & \\
HS & 1.21 & $0.41-3.63$ \\
$>$ HS & 0.78 & $0.34-1.82$ \\
Have insurance & & \\
$\quad$ No & $\mathbf{0 . 3 7}$ & $\mathbf{0 . 1 5 - 0 . 9 1}$ \\
Yes & & \\
Taking insulin & & \\
No & 2.21 & $1.10-4.84$ \\
Yes & & \\
BMI category & 1.00 & $0.27-2.24$ \\
Under/normal weight & 0.77 & $0.16-1.10$ \\
Overweight & 0.42 & \\
Obese & &
\end{tabular}

The Sobel test for mediation was used to test for the indirect effect of work hours on suboptimal and borderline glycemic control through the number of visits to a healthcare provider. This test was not statistically significant for suboptimal or borderline glycemic control (test statistic $1.294,[p=0.195]$ and $0.744[p=0.456]$ ) or for the indirect effect of farm worker occupation on suboptimal glycemic control (test statistic 1.817, $[\mathrm{p}=0.069])$.

$\underline{\text { Summary of results of research question } 1}$

In summary, it was found that diabetics working greater than 40 hours per week, relative to those working 1-20 hours per week, had greater odds of having suboptimal and borderline glycemic control, after adjusting for potential confounders. Farm workers, relative to white collar workers, were also at greater odds for suboptimal glycemic control, after adjusting for potential confounders. In addition, being a non-Hispanic Black, compared to non-Hispanic white, and taking insulin as opposed to not taking 
insulin, were risk factors for suboptimal, borderline, and poor glycemic control.

However, secondhand smoke exposure among non-smokers and desire to lose weight were not found to be statistically significant with glycemic control.

The association between glycemic control and HRQOL

Part of the second research question in this study was whether suboptimal, borderline, and poor glycemic control were associated with HRQOL measures indicative of poor health (i.e., greater days of poor physical and mental health and inactivity and poor self-reported general health). It was hypothesized that suboptimal, borderline, and poor glycemic control would be inversely associated with all four HRQOL measures, after controlling for potential confounders.

Cross-tabulations for each of the four HRQOL measures and each of the three glycemic control variables and the corresponding chi-square test statistics are shown in Table 8. The univariable multinomial regression analyses for number days of poor mental health, physical health and activity limitation are presented in Table 9. The univariable analyses for the self-reported general health variable are reported separately (i.e., not in Table 9) because it is a dichotomous variable requiring logistic regression as opposed to the other HRQOL variables that require multinomial logistic regression due to the three categories for each variable. 
Table 8. Cross-tabulations ${ }^{\dagger}$ of health related quality of life variables and suboptimal (HbA1c $\geq$ 7.0\%), borderline (HbA1c $\geq 7.0-8.9 \%)$, and poor $(\mathrm{HbA1c} \geq 9.0 \%$ ) glycemic control, adults classified with type 2 diabetes, NHAN̄ES 1999-2004

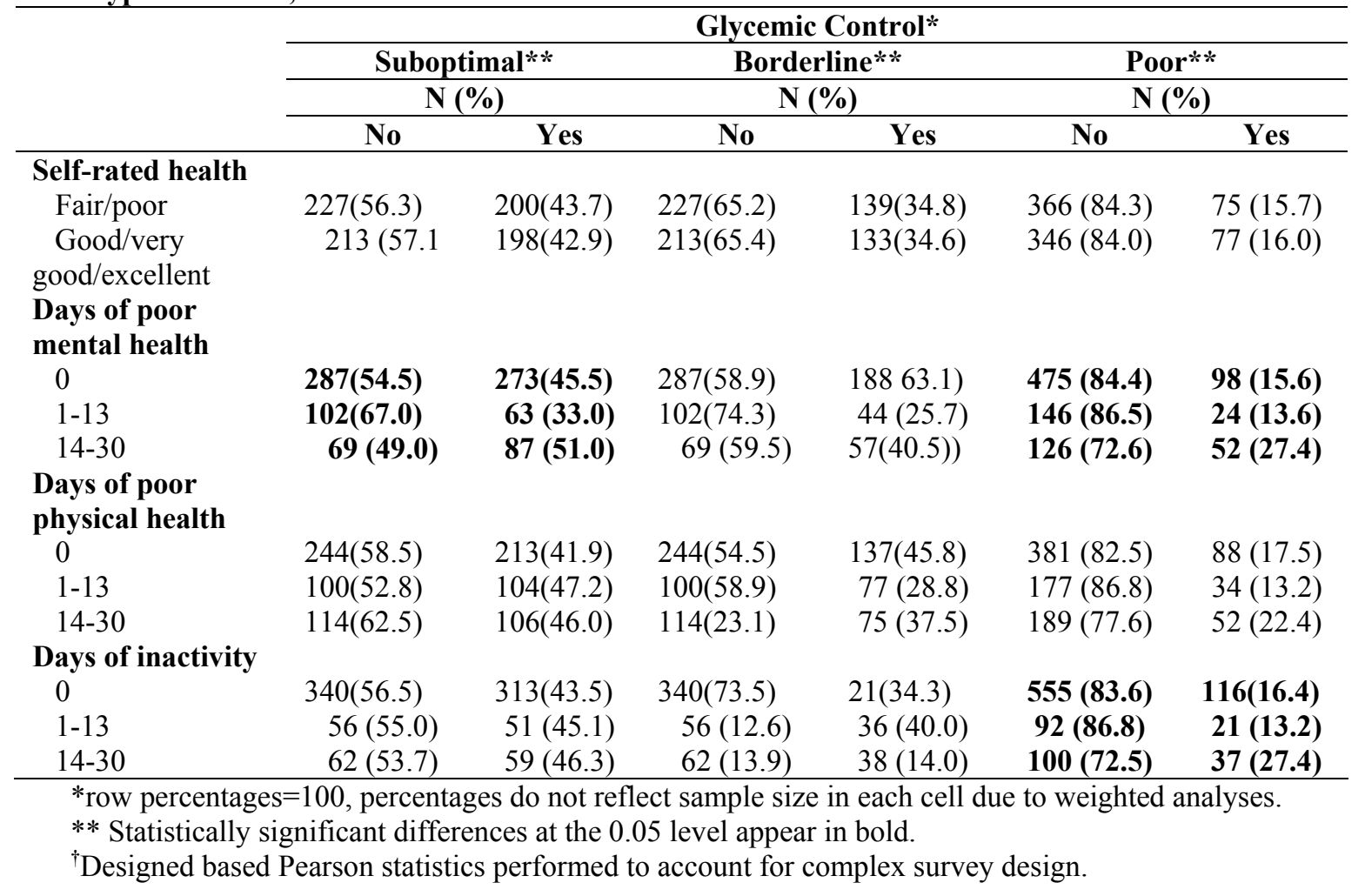

$\underline{\text { Self-rated general health }}$

As shown in the cross-tabulations distributions (table.8), self-rated health groups did not differ significantly with respect to suboptimal, borderline, and poor glycemic control. In addition, in the univariable logistic regression models, neither suboptimal glycemic control $(\mathrm{OR}=0.97[\mathrm{CI}=0.67-1.40])$, borderline $(\mathrm{OR}=0.99[\mathrm{CI}=0.67-1.47])$, or poor glycemic control $(\mathrm{OR}=1.02[\mathrm{CI}=0.62-1.67])$, were associated with fair/poor selfrated general health (versus excellent, very good, or good health) among adults with type 2 diabetes sampled from 2001-2004. 
Days of poor mental health in the past 30 days

As shown in the cross-tabulations (table 8), the proportions of suboptimal, borderline, and poor glycemic control were significantly different among poor mental health groups. In the univariable models, suboptimal, and poor glycemic control were each associated with greater number of days of poor mental health days among adults with type 2 diabetes who were 20 years of age or older sample in NHANES 2001-2004 (table 9). Specifically, suboptimal glycemic control (OR=0.59 [CI=0.38-0.92]) was associated with lower odds of having 1-13 days of poor mental health versus ' 0 days of poor mental health in the past 30 days. However, poor glycemic control was associated with greater odds of having 14 days or more versus 0 days of poor health in the past 30 days $(\mathrm{OR}=2.04[\mathrm{CI}=1.34-3.11])$. In addition, borderline glycemic control was marginally associated with $1-13$ days of poor mental health $(\mathrm{OR}=0.59[\mathrm{CI}=0.36-1.00)$. Since there was marginal significance for the relationship between borderline glycemic control and poor mental health, further multinomial logistic regression analyses were conducted adjusting for potential confounders.

Table 9. Multinomial logistic regression. Relationship between days of poor physical and mental health and activity limitation among adults with type 2 diabetes with suboptimal $(\mathrm{HbA1c} \geq 7.0 \%)$, borderline (HbA1c $\geq 7.0-8.9 \%$ ), and poor (HbA1c $\geq 9.0 \%$ ) glycemic control, NHANES 2001-2004

\begin{tabular}{|c|c|c|c|c|c|c|}
\hline \multirow[b]{4}{*}{ Characteristic } & \multicolumn{6}{|c|}{ Days of } \\
\hline & \multicolumn{2}{|c|}{ Poor Physical Health } & \multicolumn{2}{|c|}{ Poor Mental Health } & \multicolumn{2}{|c|}{ Limited Activity } \\
\hline & 1-13 days & $\begin{array}{l}>=14 \\
\text { days }\end{array}$ & 1-13 days & $>=14$ days & 1-13 days & $>=14$ days \\
\hline & $\begin{array}{c}\text { OR } \\
(95 \% \text { CI }) \\
\end{array}$ & $\begin{array}{c}\text { OR } \\
(95 \% \text { CI }) \\
\end{array}$ & $\begin{array}{c}\text { OR } \\
(95 \% \text { CI })\end{array}$ & $\begin{array}{c}\text { OR } \\
(95 \% \text { CI })\end{array}$ & $\begin{array}{c}\text { OR } \\
(95 \% \text { CI })\end{array}$ & $\begin{array}{c}\text { OR } \\
(95 \% \text { CI })\end{array}$ \\
\hline $\begin{array}{l}\text { Suboptimal } \\
\text { glycemic } \\
\text { control }\end{array}$ & & & & & & \\
\hline No & 1.00 & 1.00 & 1.00 & 1.00 & 1.00 & 1.00 \\
\hline Yes & $\begin{array}{c}1.18 \\
(0.84- \\
1.66)\end{array}$ & $\begin{array}{l}1.23 \\
(0.78- \\
1.95)\end{array}$ & $\begin{array}{c}0.59 \\
(0.89-0.92)\end{array}$ & $\begin{array}{c}1.25 \\
(0.89-1.74)\end{array}$ & $\begin{array}{c}1.06 \\
(0.63-1.78)\end{array}$ & $\begin{array}{c}1.12 \\
(0.65-1.93)\end{array}$ \\
\hline
\end{tabular}




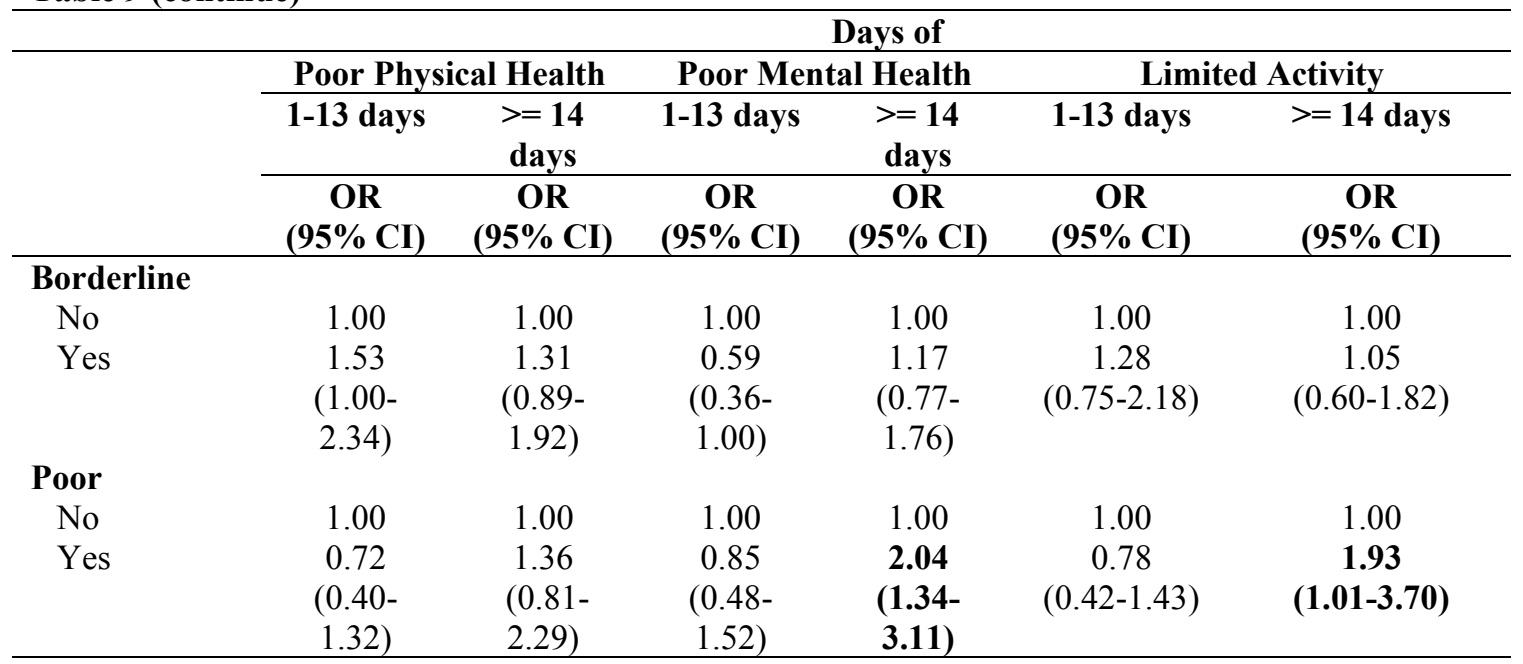

Note: 0 days of poor physical, mental health or activity limitation is the reference category.

Multivariable model development: For the models built to assess the relationship between number of days of poor physical health and all of the three glycemic control variables, the variables were entered in this multinomial logistic regression model in the following order: 1) sex, 2) age group, 3) race/ethnicity, 4) education, 5) employment status, 6) insurance status, and 7) taking insulin. The order for the model was based on adding variables that had the greatest magnitude in the association with glycemic control in the univariable model. Only the variables statistically associated with both day of poor physical health and glycemic control and that had clinical significance were entered in the model. There were no meaningful changes in the direction, strength or significance of the odds ratio of the glycemic control variables or the other covariates added at each point that a covariate was entered.

Table 10 shows the final multivariable multinomial logistic regression model for the relationship between suboptimal glycemic control and poor mental health. Adults with type 2 diabetes who had suboptimal glycemic control were less likely to have $\geq 1-13$ 
days versus 0 days of poor mental health compared to those with good glycemic control $(\mathrm{OR}=0.50[\mathrm{CI}=0.30-0.83])$, after controlling for sex, age group, race/ethnicity, education, insurance, employment status and taking insulin.

Table 10. Multinomial logistic regression. Relationship between suboptimal glycemic control (HbA1c $\geq 7.0 \%$ ) and days with poor mental health among adults with type 2 diabetes, NHANES 2001-2004

\begin{tabular}{|c|c|c|c|c|}
\hline \multirow{4}{*}{$\begin{array}{l}\text { Characteristics } \\
\text { Suboptimal } \\
\text { glycemic control }\end{array}$} & \multicolumn{4}{|c|}{ Days of poor mental health } \\
\hline & \multicolumn{2}{|c|}{ 1-13 days } & \multicolumn{2}{|c|}{$\geq 14$ days } \\
\hline & OR & $95 \%$ CI & OR & 95\% CI \\
\hline & & & & \\
\hline No & 1.00 & & 1.00 & \\
\hline Yes & $\mathbf{0 . 5 0}$ & $0.30-0.83$ & 1.03 & $0.67-1.60$ \\
\hline \multicolumn{5}{|l|}{ Sex } \\
\hline Male & 1.00 & & 1.00 & \\
\hline Female & 1.07 & $0.69-1.67$ & 1.53 & $0.86-2.72$ \\
\hline \multicolumn{5}{|l|}{ Age group (yrs) } \\
\hline $20-44$ & 1.00 & & 1.00 & \\
\hline $45-64$ & 1.29 & $0.65-2.55$ & 0.90 & $0.41-2.01$ \\
\hline $65+$ & 0.49 & $0.20-1.19$ & 0.20 & 0.08-0.47 \\
\hline \multicolumn{5}{|l|}{ Race/Ethnicity } \\
\hline NH White & 1.00 & & 1.00 & \\
\hline NH Black & 0.98 & $0.57-1.67$ & 0.69 & $0.38-1.24$ \\
\hline Hispanic & 1.22 & $0.61-2.46$ & 0.43 & $0.22-0.84$ \\
\hline Other & 0.36 & $0.18-1.11$ & 0.65 & $0.21-2.03$ \\
\hline \multicolumn{5}{|l|}{ Education $^{\wedge}$} \\
\hline$<\mathrm{HS}$ & 1.00 & & 1.00 & \\
\hline HS & 1.23 & $0.63-2.38$ & 0.74 & $0.35-1.56$ \\
\hline$>\mathrm{HS}$ & 1.40 & $0.77-2.53$ & 0.43 & $0.24-0.75$ \\
\hline \multicolumn{5}{|l|}{ Employed } \\
\hline \multicolumn{5}{|l|}{ No } \\
\hline Yes & 0.79 & $0.49-1.28$ & 0.39 & $0.24-0.65$ \\
\hline \multicolumn{5}{|l|}{ Have insurance } \\
\hline No & 1.00 & & 1.00 & \\
\hline \multicolumn{5}{|l|}{ Taking Insulin } \\
\hline No & 1.00 & & 1.00 & \\
\hline Yes & 1.62 & $0.78-3.39$ & 2.07 & $1.14-3.76$ \\
\hline
\end{tabular}

Table 11 shows the final multivariable multinomial logistic regression model for the relationship between borderline glycemic control and poor mental health. Adults with type 2 diabetes who had borderline glycemic control were less likely to have $\geq 1-13$ days 
versus 0 days of poor mental health $(\mathrm{OR}=0.55[\mathrm{CI}=0.31-0.98])$, after controlling for potential confounders.

Table 11. Multinomial logistic regression. Relationship between borderline glycemic control (HbA1c 7-8.9\%) and days with poor mental health among adults with type 2 diabetes, NHANES 2001-2004

\begin{tabular}{|c|c|c|c|c|}
\hline \multirow[b]{3}{*}{ Characteristic } & \multicolumn{4}{|c|}{ Days of poor mental health } \\
\hline & \multicolumn{2}{|c|}{ 1-13 days } & \multicolumn{2}{|c|}{$\geq 14$ days } \\
\hline & OR & $(95 \% \mathrm{CI})$ & OR & $(95 \%$ CI $)$ \\
\hline \multicolumn{5}{|c|}{$\begin{array}{l}\text { Borderline glycemic } \\
\text { control }\end{array}$} \\
\hline No & 1.00 & & 1.00 & \\
\hline Yes & 0.55 & $0.31-0.98$ & 1.00 & $0.60-1.67$ \\
\hline \multicolumn{5}{|l|}{ Sex } \\
\hline Male & 1.00 & & 1.00 & \\
\hline Female & 1.30 & $0.78-2.16$ & 1.62 & $0.92-2.88$ \\
\hline \multicolumn{5}{|l|}{ Age group (yrs) } \\
\hline $20-44$ & 1.00 & & 1.00 & \\
\hline $45-64$ & 1.13 & $0.55-2.32$ & 0.74 & $0.32-1.73$ \\
\hline $65+$ & 0.42 & $0.16-1.13$ & 0.17 & 0.07-0.44 \\
\hline \multicolumn{5}{|l|}{ Race/Ethnicity } \\
\hline NH White & 1.00 & & 1.00 & \\
\hline NH Black & 0.89 & $0.52-1.52$ & 0.75 & $0.38-1.48$ \\
\hline Hispanic & 1.70 & $0.84-3.43$ & 0.52 & $0.24-1.14$ \\
\hline Other & 0.39 & $0.13-1.14$ & 0.63 & $0.19-2.10$ \\
\hline \multicolumn{5}{|l|}{ Education $^{\wedge}$} \\
\hline$<\mathrm{HS}$ & 1.00 & & 1.00 & \\
\hline HS & 1.27 & $0.61-2.61$ & 0.84 & $0.43-1.64$ \\
\hline$>\mathrm{HS}$ & 1.54 & $0.82-2.92$ & 0.51 & $0.29-0.92$ \\
\hline \multicolumn{5}{|l|}{ Employed } \\
\hline No & 1.00 & & 1.00 & \\
\hline Yes & 0.73 & $0.43-1.26$ & 0.41 & $0.21-0.80$ \\
\hline \multicolumn{5}{|l|}{ Have insurance } \\
\hline No & 1.00 & & 1.00 & \\
\hline Yes & 0.94 & $0.36-2.48$ & 2.18 & $0.89-5.36$ \\
\hline \multicolumn{5}{|l|}{ Taking insulin } \\
\hline No & & & & \\
\hline Yes & 1.43 & $0.59-3.48$ & 1.94 & 1.01-3.74 \\
\hline
\end{tabular}

Note: 0 days of poor physical, mental health or activity limitation is the reference category.

${ }^{\wedge} \mathrm{HS}=$ High School

Table 12 shows the final multivariable multinomial logistic regression model for the relationship between poor glycemic control and number of days of poor mental health. Adults with type 2 diabetes who had poor glycemic control were more likely to 
have $\geq 14$ days versus 0 days of poor mental health compared to those with good glycemic control (OR=1.73 [CI=1.14-2.64]), after controlling for sex, age group, race/ethnicity, education, insurance, employment status and taking insulin. Although not statistically significant but similar to results using the other glycemic control variables, adults with poor glycemic control were less likely to have 1-13 days (versus 0 days) of poor mental health compared to those with good glycemic control.

Table 12. Multinomial logistic regression. Relationship between poor glycemic control (HbA1c $\geq$ 9.0\%) and days with poor mental health among adults with type 2 diabetes, NHANES 2001-2004

\begin{tabular}{|c|c|c|c|c|}
\hline \multirow[b]{3}{*}{ Characteristic } & \multicolumn{4}{|c|}{ Days of poor mental health } \\
\hline & \multicolumn{2}{|c|}{ 1-13 days } & \multicolumn{2}{|c|}{$>=14$ days } \\
\hline & OR & $(95 \% \mathrm{CI})$ & $\mathbf{O R}$ & $(95 \% \mathrm{CI})$ \\
\hline \multicolumn{5}{|l|}{$\begin{array}{l}\text { Poor glycemic } \\
\text { control }\end{array}$} \\
\hline No & 1.00 & & 1.00 & \\
\hline Yes & 0.69 & $0.38-1.26$ & 1.73 & $1.14-2.64$ \\
\hline \multicolumn{5}{|l|}{ Sex } \\
\hline Male & 1.00 & & 1.00 & \\
\hline Female & 1.08 & $0.71-1.64$ & 1.65 & $1.03-2.65$ \\
\hline \multicolumn{5}{|l|}{ Age group (yrs) } \\
\hline $20-44$ & 1.00 & & 1.00 & \\
\hline $45-64$ & 1.25 & $0.62-2.53$ & 0.72 & $0.38-1.37$ \\
\hline $65+$ & 0.50 & $0.21-1.16$ & 0.69 & 0.08-0.36 \\
\hline \multicolumn{5}{|l|}{ Race/Ethnicity } \\
\hline NH White & 1.00 & & 1.00 & \\
\hline NH Black & 0.90 & $0.55-1.46$ & 0.69 & $0.40-1.19$ \\
\hline Hispanic & 1.24 & $0.64-2.42$ & 0.46 & 0.24-0.90 \\
\hline Other & 0.30 & $0.10-0.93$ & 0.66 & $0.24-1.81$ \\
\hline \multicolumn{5}{|l|}{ Education $^{\wedge}$} \\
\hline$<\mathrm{HS}$ & 1.00 & & 1.00 & \\
\hline HS & 1.22 & $0.61-2.45$ & 0.66 & $0.33-1.32$ \\
\hline$>\mathrm{HS}$ & 1.64 & $0.90-3.00$ & 0.44 & $0.25-0.76$ \\
\hline \multicolumn{5}{|l|}{ Employed } \\
\hline \multicolumn{5}{|l|}{ No } \\
\hline Yes & 0.77 & $0.48-1.25$ & 0.41 & 0.25-0.69 \\
\hline \multicolumn{5}{|l|}{ Have insurance } \\
\hline No & 1.00 & & 1.00 & \\
\hline Yes & 0.91 & $0.39-2.10$ & 1.54 & $0.70-3.39$ \\
\hline \multicolumn{5}{|l|}{ Taking insulin } \\
\hline No & 1.00 & & 1.00 & \\
\hline Yes & 1.37 & $0.68-2.79$ & 2.32 & $1.51-3.58$ \\
\hline
\end{tabular}

Note: 0 days of poor physical, mental health or activity limitation is the reference category.

${ }^{\wedge}$ HS=High School 


\section{Days of poor physical health in the past 30 days}

As shown in the cross-tabulations (table 8), the proportions of days of poor physical health were not statistically significantly different among the various glycemic control groups. In addition, in univariable models, neither suboptimal nor poor glycemic control was associated with number of days with poor physical health (table 9). However, borderline glycemic control was marginally associated with poor physical health at the 0.20 alpha level. Specifically, borderline glycemic control was associated with greater odds of having 1-13 days of poor physical health versus 0 days of poor physical health in the past 30 days $(\mathrm{OR}=1.53[\mathrm{CI}=1.00-2.34]), \mathrm{p}=0.05)$. Since there was marginal significance for borderline glycemic control, further multinomial logistic regression analyses were conducted adjusting for potential confounders.

Multivariable model development: For the model to assess the relationship between the number of days of poor physical health and borderline glycemic control, the variables were entered in this multinomial logistic regression model in the following order: 1) sex, 2) age group, 3) race/ethnicity 4) education, 5) employment status, 6) insurance status, and 7) taking insulin. The order for the model was based on adding variables that had the greatest magnitude in the association with glycemic control in the univariable model. Only the variables statistically associated with both number of days of poor physical health and glycemic control and that had clinical significance were entered in the model. There were no meaningful changes in the direction, strength or significance of the odds ratio for the borderline glycemic control variable or the other covariates added at each point a covariate was entered. 
Table 13 shows the final multivariable multinomial logistic regression model assessing the relationship between borderline glycemic control and number of days of poor physical health. Adults with type 2 diabetes who had borderline glycemic control were statistically more likely to have 1-13 days versus 0 days of poor physical health compared to those with good control $(\mathrm{OR}=1.59[\mathrm{CI}=1.03-2.45])$; the relationship was however not statistically significant for having at least 14 days of poor physical health $(\mathrm{OR}=1.30[\mathrm{CI}=0.81-2.07])$, after controlling for sex, age group, race/ethnicity, education, insurance, employment status, and taking insulin. Being employed was protective against having $\geq 14$ days versus 0 days of poor physical health $(\mathrm{OR}=0.30[\mathrm{CI}=0.16-0.57])$.

Table 13. Multinomial logistic regression. Relationship between borderline glycemic control (HbA1c 7-8.9\%) and days with poor physical health among adults with type 2 diabetes, NHANES 2001-2004

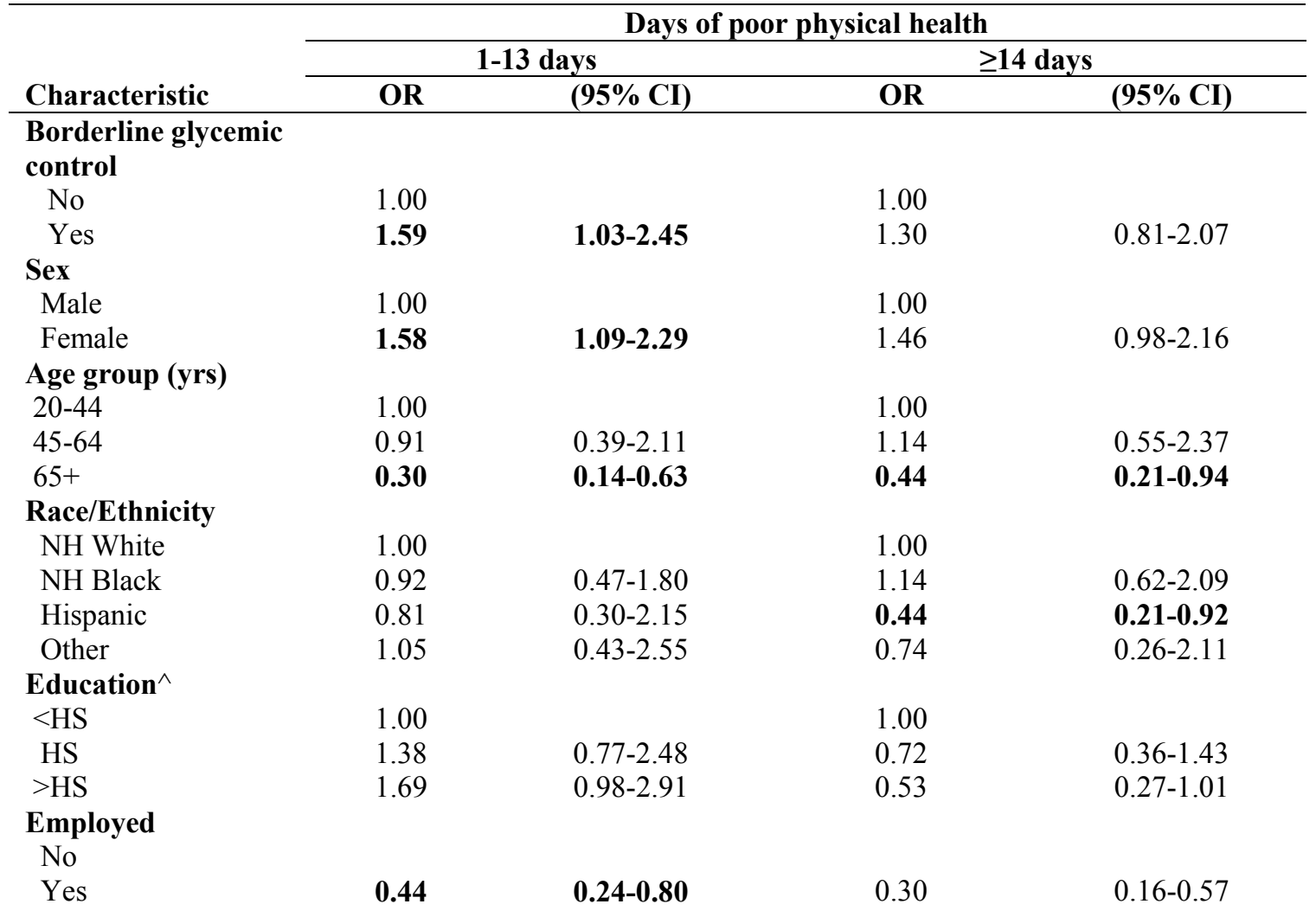


Table 13 (continue)

\begin{tabular}{|c|c|c|c|c|}
\hline & \multicolumn{2}{|c|}{ 1-13 days } & \multicolumn{2}{|c|}{$\geq 14$ days } \\
\hline Characteristic & OR & $(95 \%$ CI $)$ & OR & $(95 \%$ CI $)$ \\
\hline \multicolumn{5}{|l|}{ Have insurance } \\
\hline No & 1.00 & & 1.00 & \\
\hline Yes & 0.69 & $0.28-1.70$ & 1.30 & $0.59-2.79$ \\
\hline \multicolumn{5}{|l|}{ Taking insulin } \\
\hline No & 1.00 & & 1.00 & \\
\hline Yes & 1.83 & $0.85-3.96$ & 1.44 & $0.73-2.85$ \\
\hline
\end{tabular}

Days of inactivity due to poor health in the past 30 days

As shown in the cross-tabulations (table 8) proportions of suboptimal, borderline, or poor glycemic control were not significantly different among days of inactivity groups. In univariable models, while suboptimal or borderline glycemic control was not associated with number of inactivity days, poor glycemic control was. Specifically, among adults with type 2 diabetes, those with poor glycemic control were more likely to have $\geq 14$ days versus 0 days of inactivity $(\mathrm{OR}=1.93[\mathrm{CI}=1.01-3.70])$ (table 9).

Multivariable model development: For the model to assess the relationship between number of days of inactivity and poor glycemic control the order of how variables were entered in this multinomial logistic regression model was as follows: 1) sex, 2) age group, 3) race/ethnicity, 4) education, 5) employment status, 6) insurance status, and 7) taking insulin. The order for the model was based on adding variables that had the greatest magnitude in the association with glycemic control in the univariable model. Only the variables statistically associated with both inactivity days and glycemic control and that had clinical significance were entered in the model.

Table 14 shows the final multivariable multinomial logistic regression model assessing the relationship between number of days of inactivity and poor glycemic 
control. Adults with type 2 diabetes who had poor glycemic control were more likely to have $\geq 14$ days versus 0 days of inactivity compared to those with good control $(\mathrm{OR}=2.00[\mathrm{CI}=1.07-3.76])$, after controlling for sex, age group, race/ethnicity, education, insurance, employment status, and taking insulin. Among the confounders, being Hispanic was inversely associated with having $\geq 14$ days versus 0 days of poor physical health $(\mathrm{OR}=0.32[\mathrm{CI}=0.14-0.71])$.

Table 14. Multinomial logistic regression. Relationship between poor glycemic control (HbA1c $\geq$ 9.0\%) and inactivity days among adults with type 2 diabetes, NHANES 2001-2004

\begin{tabular}{|c|c|c|c|c|}
\hline \multirow[b]{3}{*}{ Characteristic } & \multicolumn{4}{|c|}{ Days of inactivity } \\
\hline & \multicolumn{2}{|c|}{ 1-13 days } & \multicolumn{2}{|c|}{$\geq 14$ days } \\
\hline & OR & $(95 \% \mathrm{CI})$ & OR & $(95 \% \mathrm{CI})$ \\
\hline \multicolumn{5}{|l|}{$\begin{array}{l}\text { Poor glycemic } \\
\text { control }\end{array}$} \\
\hline No & 1.00 & & 1.00 & \\
\hline Yes & 0.71 & $0.35-1.43$ & 2.00 & $1.07-3.76$ \\
\hline \multicolumn{5}{|l|}{ Sex } \\
\hline Male & 1.00 & & 1.00 & \\
\hline Female & 1.63 & $0.89-2.97$ & 1.31 & $0.81-2.14$ \\
\hline \multicolumn{5}{|l|}{ Age group (yrs) } \\
\hline $20-44$ & 1.00 & & 1.00 & \\
\hline $45-64$ & 0.58 & $0.26-1.29$ & 0.77 & $0.32-1.85$ \\
\hline $65+$ & 0.22 & $0.07-0.66$ & 0.22 & $0.09-0.53$ \\
\hline \multicolumn{5}{|l|}{ Race/Ethnicity } \\
\hline NH White & 1.00 & & 1.00 & \\
\hline NH Black & 1.03 & $0.56-1.92$ & 0.72 & $0.33-1.61$ \\
\hline Hispanic & 0.35 & 0.17-0.74 & 0.32 & 0.14-0.71 \\
\hline Other & 0.7 & $0.24-2.12$ & 0.88 & $0.36-2.14$ \\
\hline \multicolumn{5}{|l|}{ Education $^{\wedge}$} \\
\hline$<\mathrm{HS}$ & 1.00 & & 1.00 & \\
\hline HS & 0.81 & $0.43-1.50$ & 0.35 & 0.15-0.86 \\
\hline$>\mathrm{HS}$ & 0.73 & $0.31-1.69$ & 0.38 & $0.17-0.85$ \\
\hline \multicolumn{5}{|l|}{ Employed } \\
\hline \multicolumn{5}{|l|}{ No } \\
\hline Yes & 0.52 & $0.22-1.21$ & 0.22 & $0.10-0.45$ \\
\hline \multicolumn{5}{|l|}{ Have insurance } \\
\hline No & 1.00 & & 1.00 & \\
\hline Yes & 1.61 & $0.58-4.50$ & 4.01 & $1.56-10.28$ \\
\hline \multicolumn{5}{|l|}{ Taking insulin } \\
\hline No & 1.00 & & 1.00 & \\
\hline Yes & 1.83 & $0.99-3.34$ & 1.68 & $0.86-3.26$ \\
\hline
\end{tabular}

Note: 0 days of poor physical, mental health or activity limitation is the reference category.

${ }^{\wedge} \mathrm{HS}=$ High School 


\section{$\underline{\text { Missed work days and glycemic control }}$}

Part of the second research question in this study was to examine whether suboptimal, borderline, and poor glycemic control were associated with greater number of missed work days in the past 12 months among workers with type 2 diabetes. It was hypothesized that suboptimal, borderline, and poor glycemic control was associated with greater number of missed work days in the past 12 months, after controlling for potential confounders. Neither suboptimal (beta coefficient $-0.50[-1.23,-0.23]$ ), borderline glycemic control, or poor glycemic control (beta coefficient $-0.39[-0.98,-0.21]$ ), were associated with number of missed work days.

\section{Employment and glycemic control}

Part of the second research question in this study was whether suboptimal, borderline, and poor glycemic control was associated with employment among adults with type 2 diabetes. It was hypothesized that suboptimal, borderline, and poor glycemic control was inversely associated with employment, after controlling for potential confounders.

Cross-tabulations of employment and glycemic control and the corresponding Chi-square test statistics are shown in table 15. The proportions of employed versus unemployed did not vary by glycemic control groups. In univariable logistic regression, neither suboptimal glycemic control $(\mathrm{OR}=1.22[\mathrm{CI}=0.88-1.70])$ nor borderline glycemic control $(\mathrm{OR}=1.05[\mathrm{CI}=0.75-1.48])$ were significantly associated with employment. However, poor glycemic control was significantly associated with employment status $(\mathrm{OR}=1.62[\mathrm{CI}=1.05-2.53])$. 
Table 15. Cross-tabulations ${ }^{\dagger}$ of health related quality of life variables and suboptimal $(\mathrm{HbA1c} \geq$ $7.0 \%$ ), borderline (HbA1c $\geq 7.0-8.9 \%)$, and poor $(\mathrm{HbA1c} \geq \mathbf{9 . 0 \%}$ ) glycemic control among adults classified with type 2 diabetes, NHANES 1999-2004

\begin{tabular}{|c|c|c|c|c|c|c|}
\hline & \multicolumn{6}{|c|}{ Glycemic Control* } \\
\hline & \multirow{2}{*}{\multicolumn{2}{|c|}{$\frac{\text { Suboptimal** }}{\mathrm{N}(\%)}$}} & \multirow{2}{*}{\multicolumn{2}{|c|}{$\frac{\text { Borderline }^{* *}}{\mathrm{~N}(\%)}$}} & \multirow{2}{*}{\multicolumn{2}{|c|}{$\begin{array}{l}\text { Poor** } \\
\mathbf{N}(\%) \\
\end{array}$}} \\
\hline & & & & & & \\
\hline & \begin{tabular}{|l|l} 
No \\
\end{tabular} & Yes & \begin{tabular}{|l|} 
No \\
\end{tabular} & Yes & $\begin{array}{l}\text { No } \\
\end{array}$ & Yes \\
\hline \multicolumn{7}{|c|}{ Employed } \\
\hline No & 449 (52.5) & 454 (47.6) & $449(60.4)$ & 314 (39.6) & $763(86.8)$ & $140(13.2)$ \\
\hline Yes & $153(47.4)$ & $216(52.6)$ & $153(59.2)$ & $134(40.8)$ & $287(80.1)$ & $82(19.9)$ \\
\hline
\end{tabular}

\section{Multivariable model development: For the model assessing the relationship} between employment status and poor glycemic control the variables were added in the model as follows: 1) poor glycemic control, 2) sex, 3) age group, 4) race/ethnicity, 5) education, 6) insurance, 7) taking insulin, and 8) CVD status. The order for the model was based on adding variables that had the greatest magnitude in the association with glycemic control in the univariable model. Only the variables statistically associated with both employment and glycemic control and that had clinical significance were entered in the model. When age group was added in the model, the estimate for poor glycemic control changed direction, but was no longer statistically significant (0.98 [0.61-1.57]); there were no sex differences. When race/ethnicity was added, the direction of the estimates and the $p$ values for the variables in the model remained relatively unchanged except that the direction for poor glycemic control changed ( $\mathrm{OR}=1.02[\mathrm{CI}=0.65-1.63])$. Sequential addition of the other covariates (i.e. education, insurance status, taking insulin, and CVD status) did not result in any meaningful changes in the direction or significance of the estimates for poor glycemic control or the other covariates. 
Table 16 displays the final multivariable logistic regression model for the relationship between employment status and poor glycemic control. In the final adjusted model, although the results were not significant, adults with type 2 diabetes who had poor glycemic control were slightly more likely to be employed ( $\mathrm{OR}=1.10$ [CI=0.70-1.73]).

Sex specific analyses were also conducted. Females with poor glycemic control were less likely to be employed ( $\mathrm{OR}=0.84[\mathrm{CI}=0.39-1.79]$, while males with poor glycemic control were more likely to be employed ( $\mathrm{OR}=1.55[\mathrm{CI}=0.76-3.18])$.

Table 16. Multivariable logistic regression. Relationship between employment status and poor glycemic control (HbA1c $\geq 9 \%$ ) among adults with type 2 diabetes, NHANES 1999-2004

\begin{tabular}{|c|c|c|}
\hline Characteristic & Odds Ratio & 95\% Confidence Interval \\
\hline \multicolumn{3}{|c|}{ Poor glycemic control } \\
\hline \multicolumn{3}{|l|}{ Employment } \\
\hline No & 1.00 & \\
\hline Yes & 1.10 & $0.70-1.73$ \\
\hline \multicolumn{3}{|l|}{ Sex } \\
\hline Male & 1.00 & \\
\hline Female & 0.42 & $0.29-0.62$ \\
\hline \multicolumn{3}{|l|}{ Age group (yrs) } \\
\hline $20-44$ & 1.00 & \\
\hline $45-64$ & 0.44 & $0.22-0.87$ \\
\hline $65+$ & 0.05 & 0.03-0.09 \\
\hline \multicolumn{3}{|l|}{ Race/Ethnicity } \\
\hline NH White & 1.00 & \\
\hline NH Black & 0.88 & $0.52-1.48$ \\
\hline Hispanic & 1.08 & $0.54-2.17$ \\
\hline Other & 0.77 & $0.37-1.57$ \\
\hline \multicolumn{3}{|l|}{ Education $^{\wedge}$} \\
\hline$<\mathrm{HS}$ & 1.00 & \\
\hline HS & 2.10 & $1.20-3.68$ \\
\hline$>\mathrm{HS}$ & 2.46 & $1.50-4.02$ \\
\hline \multicolumn{3}{|l|}{ CVD history } \\
\hline No & 1.00 & \\
\hline Yes & 0.46 & $0.29-0.74$ \\
\hline \multicolumn{3}{|l|}{ Taking insulin } \\
\hline No & 1.00 & \\
\hline Yes & 0.63 & $0.38-1.03$ \\
\hline \multicolumn{3}{|l|}{ Have insurance } \\
\hline No & 1.00 & \\
\hline Yes & 1.12 & $0.68-1.85$ \\
\hline
\end{tabular}

$\wedge$ HS=High School 


\section{Summary of results of research question 2}

In summary, it was found that the odds having 1-13 days of poor mental health were greater among those having suboptimal and borderline glycemic control.

Individuals with poor glycemic control were more likely to report at least 14 days of poor mental health as well as inactivity in the past 30 days. In addition, greater odds for having 1-13 days of poor physical health in the past 30 days were reported among those with borderline glycemic control. However, the other measures of overall health and wellbeing, such as self-rated general health, missed work in the past 30 days and employment status, were not statistically associated with having suboptimal, borderline, or poor glycemic control, after controlling for potential confounders.

\section{$\underline{\text { Glycemic control and all-cause mortality risk }}$}

Part of the third research question was whether suboptimal, borderline, and poor glycemic control were associated with greater all-cause mortality risk among US adults with type 2 diabetes, controlling for potential confounders. It was hypothesized that individuals with suboptimal, borderline, and poor glycemic control would have greater all-cause mortality risk.

Cross-tabulations of all-cause mortality and participant characteristics including glycemic control along with the corresponding chi-square test statistics are shown in table 17. In un-adjusted Cox proportional hazards models, neither suboptimal (HR=1.22 $[\mathrm{CI}=0.95-1.58])$, borderline $(\mathrm{HR}=1.28[\mathrm{CI}=0.98-1.68])$, or poor glycemic control $(\mathrm{HR}=1.03[\mathrm{CI}=0.98-1.57])$ were associated with greater likelihood of all-cause mortality among adults with type 2 diabetes (Table 18). Factors associated with all-cause mortality 
in un-adjusted analyses included being greater than 65 years of age $(\mathrm{HR}=6.01[\mathrm{CI}=2.36$ 15.61]), being Hispanic (HR 0.59 [0.44-0.78]), being married or living with a partner $(\mathrm{HR}=1.75[\mathrm{CI}=1.29-2.36])$, cancer history $(\mathrm{HR}=1.58[\mathrm{CI}=1.05-2.38])$, being obese $(\mathrm{HR}=$ $0.54[\mathrm{CI}=0.37-0.78])$, taking insulin $(\mathrm{HR}=0.59[\mathrm{CI}=0.41-0.85])$, and being a high school graduate $(\mathrm{HR}=0.66[\mathrm{CI}=0.49-0.91])$ or having more than a high school education $(\mathrm{HR}=0.57[\mathrm{CI}=0.37-0.88])$ (Table 18). Since age is an important predictor of death, and because we wanted to assess the possibility of confounders, age and other variables were included in the adjusted model.

The covariates included in the adjusted models for each of the glycemic control variables were entered in the following order: 1) age group, 2) sex, 3) race/ethnicity, 4) education, 5) BMI category, 6) duration of diabetes, 7) cancer history, and 8) hypertension diagnosis. However, there were no differences in the association between the glycemic control variables and mortality risk after each covariate was sequentially added to the model. There were also no changes in the hazard ratios of the other covariates as each variable was added in the model.

Table 17. Cross-tabulations ${ }^{\dagger}$ of cause of death with participant characteristics, adults classified with type 2 diabetes, NHANES 1986-1994 and NHANES III mortality linked data file

\begin{tabular}{|c|c|c|c|c|}
\hline & \multicolumn{4}{|c|}{ Cause of Death } \\
\hline & \multirow{2}{*}{\multicolumn{2}{|c|}{$\begin{array}{c}\text { All-cause } \\
\mathbf{N}(\%) \\
\end{array}$}} & \multirow{2}{*}{\multicolumn{2}{|c|}{$\frac{\text { Cardiovascular }}{\mathbf{N}(\%)}$}} \\
\hline & & & & \\
\hline & No & Yes & No & Yes \\
\hline \multicolumn{5}{|l|}{$\begin{array}{l}\text { Suboptimal glycemic } \\
\text { control }\end{array}$} \\
\hline No & 296 (67.6) & $210(32.4)$ & $96(42.2)$ & $114(57.8)$ \\
\hline Yes & $402(63.5)$ & $264(36.5)$ & $137(52.3)$ & $127(47.7)$ \\
\hline
\end{tabular}


Table 17 (continue)

\begin{tabular}{|c|c|c|c|c|}
\hline & \multicolumn{4}{|c|}{ Cause of Death } \\
\hline & \multirow{2}{*}{\multicolumn{2}{|c|}{$\begin{array}{c}\text { All-cause } \\
\mathbf{N}(\%)\end{array}$}} & \multirow{2}{*}{\multicolumn{2}{|c|}{$\frac{\text { Cardiovascular }}{\mathrm{N}(\%)}$}} \\
\hline & & & & \\
\hline & No & Yes & No & Yes \\
\hline \multicolumn{5}{|l|}{$\begin{array}{l}\text { Borderline glycemic } \\
\text { control }\end{array}$} \\
\hline No & $296(67.60$ & $210(32.4)$ & $96(42.2)$ & $114(57.8)$ \\
\hline Yes & $214(62.1)$ & 149 (37.9) & $77(56.2)$ & $72(43.8)$ \\
\hline \multicolumn{5}{|l|}{$\begin{array}{l}\text { Poor glycemic } \\
\text { control }\end{array}$} \\
\hline No & $510(65.3)$ & $359(34.7)$ & $173(48.5)$ & $186(51.5)$ \\
\hline Yes & $188(65.4)$ & $115(34.6)$ & $6046.6)$ & $55(53.4)$ \\
\hline \multicolumn{5}{|l|}{ Sex } \\
\hline Male & $321(66.4)$ & 304 (33.6) & $140(42.6)$ & $164(57.4)$ \\
\hline Female & $458(64.2)$ & $296(35.8)$ & $145(52.9)$ & $151(47.1)$ \\
\hline \multicolumn{5}{|l|}{ Age group } \\
\hline $20-44$ & $114(87.7)$ & $10(12.3)$ & $8(69.0)$ & $2(31.0)$ \\
\hline $45-64$ & $355(79.9)$ & $127(20.1)$ & $72(54.1)$ & $55(45.9)$ \\
\hline $65+$ & $310(44.2)$ & $463(55.8)$ & $205(45.1)$ & $258(54.9)$ \\
\hline \multicolumn{5}{|l|}{ Race/ethnicity } \\
\hline Non-Hispanic White & $261(63.4)$ & $301(36.6)$ & $127(46.5)$ & $174(53.5)$ \\
\hline Non-Hispanic Black & $212(63.5)$ & $153(36.5)$ & $84(58.6)$ & 69 (41.4) \\
\hline Hispanic & $267(76.9)$ & 134 (23.1) & $70(58.5)$ & $64(41.5)$ \\
\hline Other & $39(81.5)$ & $12(18.5)$ & $4(36.3)$ & $8(63.7)$ \\
\hline \multicolumn{5}{|l|}{ Education $^{\wedge}$} \\
\hline$<\mathrm{HS}$ & $465(57.8)$ & $395(42.2)$ & 183(48.6) & $212(51.4)$ \\
\hline HS & $178(69.4)$ & $109(30.6)$ & $56(49.5)$ & $53(50.5)$ \\
\hline$>\mathrm{HS}$ & $130(74.4)$ & $85(26.0)$ & $39(45.4)$ & $46(54.6)$ \\
\hline \multicolumn{5}{|l|}{ Marital status } \\
\hline Not married & $506(70.8)$ & $322(29.2)$ & $145(42.7)$ & $177(57.3)$ \\
\hline $\begin{array}{l}\text { Married/living with } \\
\text { partner }\end{array}$ & $270(55.0)$ & $276(45.0)$ & $139(55.2)$ & $137(44.8)$ \\
\hline \multicolumn{5}{|l|}{ Hypertension diagnosis } \\
\hline No & $357(66.6)$ & $238(33.4)$ & $114(49.7)$ & $124(50.4)$ \\
\hline Yes & $422(64.2)$ & $359(35.8)$ & $170(47.1)$ & $189(52.9)$ \\
\hline \multicolumn{5}{|l|}{ Cancer history } \\
\hline No & $718(67.0)$ & $505(33.0)$ & $234(45.6)$ & $271(54.4)$ \\
\hline Yes & $61(53.9)$ & 95 (46.1) & $51(60.7)$ & $44(39.2)$ \\
\hline \multicolumn{5}{|l|}{ Take insulin } \\
\hline No & $197(55.2)$ & $211(44.8)$ & $132(46.5)$ & $158(53.5)$ \\
\hline Yes & $581(68.8)$ & 389 (31.2) & $153(52.3)$ & $153(47.7)$ \\
\hline \multicolumn{5}{|l|}{ BMI category } \\
\hline Normal/under weight & $122(53.4)$ & $131(46.6)$ & $65(49.3)$ & $66(50.7)$ \\
\hline Overweight & $266(64.5)$ & $208(35.5)$ & $94(41.8)$ & $114(58.2)$ \\
\hline Obese & $334(70.6)$ & $159(39.4)$ & $86(54.0)$ & $73(46.0)$ \\
\hline
\end{tabular}

*row percentages $=100$, percentages do not reflect sample size in each cell due to weighted analyses.

** Statistically significant differences at the 0.05 level appear in bold.

${ }^{\dagger}$ Designed based Pearson statistics performed to account for complex survey design.

$\wedge$ HS=High School 
Table 18. Un-adjusted Cox proportional hazards model. Factors associated with all-cause and cardiovascular mortality risk among adults with type 2 diabetes, NHANES III

\begin{tabular}{|c|c|c|c|c|}
\hline & \multicolumn{4}{|c|}{ Cause of Death } \\
\hline & \multicolumn{2}{|c|}{ All-cause } & \multicolumn{2}{|c|}{ Cardiovascular } \\
\hline & $\begin{array}{l}\text { Hazards } \\
\text { Ratio }\end{array}$ & $\begin{array}{c}95 \% \\
\text { Confidence } \\
\text { Interval }\end{array}$ & $\begin{array}{c}\text { Hazards } \\
\text { Ratio }\end{array}$ & $\begin{array}{c}95 \% \\
\text { Confidence } \\
\text { Interval }\end{array}$ \\
\hline \multicolumn{5}{|l|}{ Suboptimal glycemic control } \\
\hline No & 1.00 & & 1.00 & \\
\hline Yes & 1.22 & $0.95-1.58$ & 1.04 & $0.75-1.44$ \\
\hline \multicolumn{5}{|l|}{$\begin{array}{l}\text { Borderline glycemic } \\
\text { control }\end{array}$} \\
\hline No & 1.00 & & 1.00 & \\
\hline Yes & 1.28 & $0.98-1.68$ & 1.00 & $0.68-1.49$ \\
\hline \multicolumn{5}{|l|}{$\begin{array}{l}\text { Poor glycemic } \\
\text { control }\end{array}$} \\
\hline No & 1.00 & & 1.00 & \\
\hline Yes & 1.03 & $0.98-1.57$ & 1.09 & $0.54-2.19$ \\
\hline \multicolumn{5}{|l|}{ Sex } \\
\hline Male & 1.00 & & 1.00 & \\
\hline Female & 1.06 & $0.82-1.38$ & 0.91 & $0.67-1.23$ \\
\hline \multicolumn{5}{|l|}{ Age group } \\
\hline $20-44$ & 1.00 & & 1.00 & \\
\hline $45-64$ & 1.57 & $0.63-3.94$ & 2.14 & $0.34-13.19$ \\
\hline $65+$ & 6.01 & 2.36-15.61 & 10.56 & $1.77-63.21$ \\
\hline \multicolumn{5}{|l|}{ Race/ethnicity } \\
\hline Non-Hispanic White & 1.00 & & 1.00 & \\
\hline Non-Hispanic Black & 1.04 & $0.80-1.35$ & 0.81 & $0.58-1.12$ \\
\hline Hispanic & 0.59 & $0.44-0.78$ & 0.47 & $0.29-0.77$ \\
\hline Other & 0.53 & $0.21-1.37$ & 0.63 & $0.19-2.15$ \\
\hline \multicolumn{5}{|l|}{ Education $^{\wedge}$} \\
\hline$<\mathrm{HS}$ & 1.00 & & 1.00 & \\
\hline $\mathrm{HS}$ & 0.66 & $0.49-0.91$ & 0.57 & $0.39-0.83$ \\
\hline$>\mathrm{HS}$ & 0.57 & $0.37-0.88$ & 0.66 & 0.44-0.99 \\
\hline \multicolumn{5}{|l|}{ Marital status } \\
\hline Not married & 1.00 & & 1.00 & \\
\hline Married/living with partner & 1.75 & $1.29-2.36$ & 1.40 & $0.91-2.16$ \\
\hline \multicolumn{5}{|l|}{ Hypertension diagnosis } \\
\hline No & 1.00 & & 1.00 & \\
\hline Yes & 1.05 & $0.77-1.42$ & 1.08 & $0.74-1.58$ \\
\hline \multicolumn{5}{|l|}{ Cancer history } \\
\hline No & 1.00 & & 1.00 & \\
\hline Yes & 1.58 & 1.05-2.38 & 1.14 & $0.71-1.85$ \\
\hline \multicolumn{5}{|l|}{ Take insulin } \\
\hline No & 1.00 & & 1.00 & \\
\hline Yes & 0.59 & $0.41-0.85$ & 0.67 & $0.41-1.09$ \\
\hline Duration of diabetes & 1.03 & $1.02-1.04$ & 1.03 & 1.01-1.04 \\
\hline \multicolumn{5}{|l|}{ BMI category } \\
\hline Normal/under weight & 1.00 & & 1.00 & \\
\hline Overweight & 0.70 & $0.47-1.04$ & 0.80 & $0.51-1.24$ \\
\hline Obese & 0.54 & $0.37-0.78$ & 0.47 & $0.28-0.79$ \\
\hline
\end{tabular}




\section{$\underline{\text { Suboptimal glycemic control }}$}

The adjusted Cox proportional hazards model for the relationship between suboptimal glycemic control and all-cause mortality risk for a participants is presented in Table 19. Suboptimal glycemic control was not statistically associated with all-cause mortality risk among adults with type 2 diabetes $(\mathrm{HR}=1.24[\mathrm{CI}=0.99-1.56])$.

Table 19. Adjusted Cox proportional hazards model. Relationship between suboptimal glycemic control and all-cause mortality risk among adults with type 2 diabetes, NHANES III

\begin{tabular}{|c|c|c|}
\hline Characteristic & Hazards Ratio & 95\% Confidence Interval \\
\hline \multicolumn{3}{|l|}{ Suboptimal glycemic control } \\
\hline No & 1.00 & \\
\hline Yes & 1.24 & $0.99-1.56$ \\
\hline \multicolumn{3}{|l|}{ Age group (yrs) } \\
\hline $20-44$ & 1.00 & \\
\hline $45-64$ & 1.75 & $0.61-4.99$ \\
\hline $65+$ & 5.66 & 202-15.82 \\
\hline \multicolumn{3}{|l|}{ Sex } \\
\hline Male & 1.00 & \\
\hline Female & 0.90 & $0.68-1.18$ \\
\hline \multicolumn{3}{|l|}{ Race/Ethnicity } \\
\hline NH White & 1.00 & \\
\hline NH Black & 1.05 & $0.77-1.43$ \\
\hline Hispanic & 0.62 & $0.48-0.80$ \\
\hline Other & 0.63 & $0.24-1.63$ \\
\hline \multicolumn{3}{|l|}{ Education $^{\wedge}$} \\
\hline$<\mathrm{HS}$ & 1.00 & \\
\hline HS & 0.69 & $0.49-0.97$ \\
\hline$>\mathrm{HS}$ & 0.63 & $0.45-0.88$ \\
\hline \multicolumn{3}{|l|}{ BMI category } \\
\hline Under/normal weight & 1.00 & \\
\hline Overweight & 0.67 & $0.45-0.99$ \\
\hline Obese & 0.65 & $0.45-0.94$ \\
\hline Duration of diabetes (yrs) & 1.01 & $0.99-1.02$ \\
\hline \multicolumn{3}{|l|}{ Hypertension diagnosis } \\
\hline No & 1.00 & \\
\hline Yes & 1.07 & $0.80-1.42$ \\
\hline \multicolumn{3}{|l|}{ Cancer history } \\
\hline No & 1.00 & \\
\hline Yes & 1.21 & $0.79-1.86$ \\
\hline
\end{tabular}

$\wedge$ HS=High School 


\section{Borderline glycemic control}

The adjusted Cox proportional hazards model for the relationship between borderline glycemic control and all-cause mortality risk is presented in Table 20.

Borderline glycemic control was not statistically associated with all-cause mortality risk among adults with type 2 diabetes in un-stratified analyses ( $\mathrm{HR}=1.20$ [CI=0.94-1.53]) or in the stratified analyses $(\mathrm{HR}=1.02[\mathrm{CI}=0.61-1.71]$ and $\mathrm{HR}=1.21[\mathrm{CI}=0.78-2.86])$ for males and females, respectively.

Table 20. Adjusted Cox proportional hazards model. Relationship between borderline glycemic control and all-cause mortality risk among adults with type 2 diabetes, NHANES III

\begin{tabular}{|c|c|c|}
\hline Characteristic & Hazards Ratio & 95\% Confidence Interval \\
\hline \multicolumn{3}{|l|}{ Borderline Glycemic control } \\
\hline No & 1.00 & \\
\hline Yes & 1.20 & $0.94-1.53$ \\
\hline \multicolumn{3}{|l|}{ Age group (yrs) } \\
\hline $20-44$ & 1.00 & \\
\hline $45-64$ & 1.11 & $0.37-3.35$ \\
\hline $65+$ & 3.69 & 1.25-10.87 \\
\hline \multicolumn{3}{|l|}{ Sex } \\
\hline Male & 1.00 & \\
\hline Female & 0.96 & $0.71-1.30$ \\
\hline \multicolumn{3}{|l|}{ Race/Ethnicity } \\
\hline NH White & 1.00 & \\
\hline NH Black & 1.02 & $0.71-1.48$ \\
\hline Hispanic & 0.59 & $0.39-0.88$ \\
\hline Other & 0.46 & $0.16-1.34$ \\
\hline \multicolumn{3}{|l|}{ Education $^{\wedge}$} \\
\hline$<\mathrm{HS}$ & 1.00 & \\
\hline HS & 0.77 & 0.54-1.09 \\
\hline$>\mathrm{HS}$ & 0.60 & $0.39-0.93$ \\
\hline \multicolumn{3}{|l|}{ BMI category } \\
\hline Under/normal weight & 1.00 & \\
\hline Overweight & 0.70 & $0.45-1.08$ \\
\hline Obese & 0.70 & $0.44-0.98$ \\
\hline Duration of diabetes (yrs) & 1.01 & $1.00-1.02$ \\
\hline \multicolumn{3}{|l|}{ Hypertension diagnosis } \\
\hline No & 1.00 & \\
\hline Yes & 1.16 & $0.88-1.54$ \\
\hline \multicolumn{3}{|l|}{ Cancer history } \\
\hline No & 1.00 & \\
\hline Yes & 1.18 & $0.77-1.81$ \\
\hline
\end{tabular}

$\wedge$ HS=High School 
$\underline{\text { Poor glycemic control }}$

The adjusted Cox proportional hazards model for the relationship between poor glycemic control and all-cause mortality risk is presented in Table 21. Poor glycemic control was not statistically associated with all-cause mortality risk $(\mathrm{HR}=1.24[\mathrm{CI}=0.79$ 1.95]). Of note, addition of the interaction term of BMI category and sex did not change the results in the in the adjusted model nor was it statistically significant when added in the model.

Table 21. Adjusted Cox proportional hazards model. Relationship between poor glycemic control and all-cause mortality risk among adults with type 2 diabetes, NHANES III

\begin{tabular}{|c|c|c|}
\hline Characteristic & Hazards Ratio & 95\% Confidence Interval \\
\hline \multicolumn{3}{|l|}{ Poor glycemic control } \\
\hline No & 1.00 & \\
\hline Yes & 1.24 & $0.79-1.96$ \\
\hline \multicolumn{3}{|l|}{ Age group (yrs) } \\
\hline $20-44$ & 1.00 & \\
\hline $45-64$ & 1.81 & $0.62-5.20$ \\
\hline $65+$ & 5.89 & $2.10-16.54$ \\
\hline \multicolumn{3}{|l|}{ Sex } \\
\hline Male & 1.00 & \\
\hline Female & 0.89 & $0.68-1.17$ \\
\hline \multicolumn{3}{|l|}{ Race/Ethnicity } \\
\hline NH White & 1.00 & \\
\hline NH Black & 1.03 & $0.77-1.41$ \\
\hline Hispanic & 0.63 & $0.49-0.81$ \\
\hline Other & 0.62 & $0.24-1.58$ \\
\hline \multicolumn{3}{|l|}{ Education $^{\wedge}$} \\
\hline$<\mathrm{HS}$ & 1.00 & \\
\hline HS & 0.71 & $0.50-0.99$ \\
\hline$>\mathrm{HS}$ & 0.63 & $0.45-0.89$ \\
\hline \multicolumn{3}{|l|}{ BMI category } \\
\hline Under/normal weight & 1.00 & \\
\hline Overweight & 0.67 & $0.45-1.00$ \\
\hline Obese & 0.64 & $0.44-0.93$ \\
\hline Duration of diabetes (yrs) & 1.01 & $0.99-1.02$ \\
\hline \multicolumn{3}{|l|}{ Hypertension diagnosis } \\
\hline No & 1.00 & \\
\hline Yes & 1.06 & $0.79-1.42$ \\
\hline \multicolumn{3}{|l|}{ Cancer history } \\
\hline No & 1.00 & \\
\hline Yes & 1.19 & $0.76-1.85$ \\
\hline
\end{tabular}

${ }^{\wedge}$ HS=High School 
Sex-specific analyses were also conducted. In the sex-specific analyses, suboptimal glycemic control was not associated with all-cause mortality among males $(\mathrm{HR}=1.19[\mathrm{CI}=0.80-1.77]$.$) nor females (\mathrm{HR}=1.19[\mathrm{CI}=0.77-1.83])$. In addition, there were no statistically significant relationship between all-cause mortality risk and borderline glycemic control among males $(\mathrm{HR}=1.02[\mathrm{CI}=0.61-1.71])$ nor females $(\mathrm{HR}=1.21[\mathrm{CI}=0.78-2.86])$. In addition, all-cause mortality risk was not associated with poor glycemic control among males $(\mathrm{HR}=1.45[\mathrm{CI}=0.88-2.39]$ nor females $(\mathrm{HR}=1.09$ $[\mathrm{CI}=0.59-2.00])$. Furthermore, when using the continuous variable of $\mathrm{HbAlc}$ values as the independent variables, levels of $\mathrm{HbAlc}$ were not found to be statistically significantly associated with all-cause mortality $(\mathrm{HR}=1.06[\mathrm{CI}=0.98-1.15])$. However, when stratifying by sex, a statistically significant association with all-cause mortality risk was found for males $(\mathrm{HR}=1.14[\mathrm{CI}=1.02-1.28])$ but not for females $(\mathrm{HR}=1.05[\mathrm{CI}=0.94-1.16])$ in multivariable analyses. There was no statistically significant relationship between HbAlc values and CVD mortality risk. Sex-specific analyses for CVD mortality were not possible due to the few CVD deaths.

Further analyses were also conducted using different HbA1c cut-offs. Specifically, the thresholds of $6.5 \%$ and $6.0 \%$ we used. Diabetic individuals with a HbAlc of less than $6.0 \%$ or $6.5 \%$ had a statistically significant lower mortality risk (HR 0.69 [0.48-99] and 0.72 [0.57-0.92], respectively) compared to individuals with greater values, even after adjusting for potential confounders. The HbAlc threshold of $6.5 \%$ was used since it is a cut-off used by AACE for suboptimal glycemic control. ${ }^{13}$ Additional hazard analyses were conducted among four diabetic groups. Specifically, these groups were the following: 1) 65 years of age or older $(n=620), 2) 65$ years of age or older and 
taking insulin only ( $\mathrm{n}=169), 3) 65$ years of age or older, taking insulin only, 4) 65 years of age or older and being diagnosed with hypertension (n-344), 5) taking insulin only ( $\mathrm{n}=$ 332), and 6) being diagnosed with hypertension ( $\mathrm{n}=644)$. Among these diabetic groups, statistically significant relationships between strict glycemic control and all-cause mortality risk were not found. Specifically, diabetics 65 years of age or older with an $\mathrm{HbA} 1 \mathrm{c}$ of less than $6.5 \%$ had a mortality risk of $0.73[\mathrm{CI}=0.48-1.12]$; diabetics 65 years of age or older and taking insulin only with a HbAlc of less than $6.5 \%$ had a mortality risk of $1.05[\mathrm{CI}=0.37-3.00]$; diabetics 65 years of age or older and taking insulin only and diagnosed with hypertension with an $\mathrm{HbA} 1 \mathrm{c}$ of less than $6.5 \%$ had a mortality risk of $1.41[\mathrm{CI}=0.51-3.88] ; 4)$ diabetics 65 years of age or older and diagnosed with hypertension with an $\mathrm{HbAlc}$ of less than $6.5 \%$ had a mortality risk of 0.87 [CI $=0.64-$ 1.22]); 5) diabetics taking insulin only with an $\mathrm{HbAlc}$ of less than $6.5 \%$ had a mortality risk of 0.95 [CI=0.46-1.97]; and 6) diabetics diagnosed with hypertension and with an HbA1c of less than $6.5 \%$ had mortality risk of $0.84[\mathrm{CI}=0.52-1.36]$.

\section{Glycemic control and cardiovascular mortality risk}

Part of the third research question of this study was whether suboptimal, borderline, and poor glycemic control were associated with greater cardiovascular mortality risk among US adults with type 2 diabetes, after controlling for potential confounders. It was hypothesized that individuals with suboptimal, borderline, and poor glycemic control would have greater cardiovascular mortality risk.

Cross-tabulations of cardiovascular mortality and participant characteristics including glycemic control along with the corresponding chi-square test statistics are 
shown in the table 17. In un-adjusted Cox proportional hazards models, neither suboptimal $(\mathrm{HR}=1.04[\mathrm{CI}=0.75-1.44])$, borderline $(\mathrm{HR}=1.0[\mathrm{CI}=0.68-1.49])$, or poor glycemic control $(\mathrm{HR}=1.09[\mathrm{CI}=0.54-2.19])$ were associated with greater likelihood of cardiovascular mortality among adults with type 2 diabetes (Table 18). To be consistent with the hazard models for all-cause mortality, adjusted hazards models were also performed with the same variables added in the model except for cancer diagnosis. However, there were no differences in the association between the glycemic control variables and CVD mortality risk after a covariate was added in the model.

\section{Suboptimal glycemic control}

The adjusted Cox proportional hazards model for the relationship between suboptimal glycemic control and CVD mortality risk is presented in Table 22.

Suboptimal glycemic control was not statistically associated with CVD mortality risk among adults with type 2 diabetes $(\mathrm{HR}=1.06[\mathrm{CI}=0.75-1.51])$.

\begin{tabular}{|c|c|c|}
\hline Characteristic & Hazards Ratio & 95\% Confidence Interval \\
\hline \multicolumn{3}{|c|}{ Suboptimal glycemic control } \\
\hline No & 1.00 & \\
\hline Yes & 1.06 & $0.75-1.51$ \\
\hline \multicolumn{3}{|l|}{ Age group (yrs) } \\
\hline $20-44$ & 1.00 & \\
\hline $45-64$ & 1.69 & $0.26-10.84$ \\
\hline $65+$ & 6.92 & $1.12-42.69$ \\
\hline \multicolumn{3}{|l|}{ Sex } \\
\hline Male & 1.00 & \\
\hline Female & 0.77 & $0.51-1.18$ \\
\hline \multicolumn{3}{|l|}{ Race/Ethnicity } \\
\hline NH White & 1.00 & \\
\hline NH Black & 0.78 & $0.53-1.16$ \\
\hline
\end{tabular}


Table 22 (continue)

\begin{tabular}{lcc}
\hline Characteristic & Hazards Ratio & $\mathbf{9 5 \%}$ Confidence Interval \\
\hline Hispanic & $\mathbf{0 . 4 9}$ & $\mathbf{0 . 2 9 - 0 . 8 3}$ \\
Other & $\mathbf{0 . 8 6}$ & $\mathbf{0 . 2 9 - 2 . 5 7}$ \\
Education & 1.00 & \\
<HS & 0.64 & $0.40-1.05$ \\
HS & 0.63 & $0.40-1.00$ \\
>HS & & \\
BMI category & & \\
Under/normal weight & 0.74 & $0.40-1.13$ \\
Overweight & $\mathbf{0 . 5 7}$ & $\mathbf{0 . 3 6 - 0 . 8 9}$ \\
Obese & 1.01 & $0.99-1.02$ \\
Duration of diabetes (yrs) & & \\
Hypertension diagnosis & 1.00 & $0.76-1.72$ \\
$\quad$ No & 1.14 & \\
Yes & & \\
\hline
\end{tabular}

Borderline glycemic control

The adjusted Cox proportional hazards model for the relationship between borderline glycemic control and CVD mortality risk is presented in Table 23 . Borderline glycemic control was not statistically associated with CVD mortality risk among adults with type 2 diabetes $(\mathrm{HR}=0.91[\mathrm{CI}=0.62-1.33])$.

Table 23. Adjusted Cox proportional hazards model. Relationship between borderline glycemic control and CVD mortality risk among adults with type 2 diabetes, NHANES III

\begin{tabular}{lcc}
\hline Characteristic & Hazards Ratio & $\mathbf{9 5 \%}$ Confidence Interval \\
\hline Borderline glycemic control & 1.00 & \\
$\quad$ No & 0.91 & $0.62-1.33$ \\
$\quad$ Yes & 1.00 & \\
Age group (yrs) & 1.10 & $0.17-7.33$ \\
$20-44$ & 4.30 & $0.73-25.34$ \\
$45-64$ & & \\
$65+$ & 1.00 & \\
Sex & 0.77 & \\
Male & & \\
Female & 1.00 & \\
Race/Ethnicity & 0.72 & $0.45-1.24$ \\
NH White & 0.50 & $0.25-1.01$ \\
NH Black & 0.48 & $0.12-2.08$ \\
Hispanic & & \\
Other &
\end{tabular}


Table 23 (continue)

\begin{tabular}{lcc}
\hline Characteristic & Hazards Ratio & 95\% Confidence Interval \\
\hline Education $^{\wedge}$ & 1.00 & \\
<HS & 0.79 & $0.47-1.35$ \\
HS & 0.62 & $0.33-1.16$ \\
>HS & & \\
BMI category & 1.00 & \\
$\quad$ Under/normal weight & 0.80 & $0.49-1.29$ \\
$\quad$ Overweight & 0.60 & $0.34-1.04$ \\
$\quad$ Obese & 1.01 & $0.99-1.03$ \\
Duration of diabetes $($ yrs) & & \\
Hypertension diagnosis & 1.00 & \\
No & 1.19 & $0.75-1.90$ \\
$\quad$ Yes & & \\
\hline
\end{tabular}

${ }^{\wedge} \mathrm{HS}=$ High School

$\underline{\text { Poor glycemic control }}$

The adjusted Cox proportional hazards model for the relationship between poor glycemic control and CVD mortality risk is presented in Table 24. Poor glycemic control was not statistically associated with CVD mortality risk in un-stratified analyses

$(\mathrm{HR}=1.38[\mathrm{CI}=0.67-2.82])$.

Table 24. Adjusted Cox proportional hazards model. Relationship between poor glycemic control and CVD mortality risk among adults with type 2 diabetes,

NHANES III

\begin{tabular}{lcc}
\hline Characteristic & Hazards Ratio & $\mathbf{9 5 \%}$ Confidence Interval \\
\hline $\begin{array}{l}\text { Poor glycemic control } \\
\text { No }\end{array}$ & 1.00 & \\
Yes & 1.38 & $0.67-2.82$ \\
Age group (yrs) & & \\
20-44 & 1.00 & \\
$45-64$ & 1.76 & $0.27-11.22$ \\
$65+$ & $\mathbf{7 . 2 5}$ & $\mathbf{1 . 1 4 - 4 5 . 9 0}$ \\
Sex & & \\
Male & 1.00 & $0.50-1.16$ \\
Female & 0.76 & \\
Race/Ethnicity & & \\
NH White & 1.00 & $0.52-1.15$ \\
NH Black & 0.77 & $\mathbf{0 . 3 0 - 0 . 8 1}$ \\
Hispanic & $\mathbf{0 . 4 9}$ & $0.29-2.59$ \\
Other & 0.87 & \\
Education \\
$<$ HS & &
\end{tabular}


Table 24 (continue)

\begin{tabular}{lcc}
\hline Characteristic & Hazards Ratio & $\mathbf{9 5 \%}$ Confidence Interval \\
\hline HS & 0.65 & $0.41-1.06$ \\
> HS & 0.64 & $0.40-1.01$ \\
BMI category & 1.00 & \\
Under/normal weight & 0.75 & $0.48-1.15$ \\
Overweight & $\mathbf{0 . 5 6}$ & $\mathbf{0 . 3 6 - 0 . 8 9}$ \\
Obese & 1.01 & $0.99-1.02$ \\
Duration of diabetes (yrs) & & \\
Hypertension diagnosis & 1.00 & $0.75-1.72$ \\
No & 1.13 & \\
Yes & &
\end{tabular}

${ }^{\wedge}$ HS $=$ High School

$\underline{\text { Summary of results of research question } 3}$

In summary, it was found that neither all-cause nor CVD mortality risk was statistically associated with having suboptimal, borderline, or poor glycemic control, after controlling for potential confounders. However, when $\mathrm{HbA} 1 \mathrm{c}$ was used as a continuous variable, males with greater $\mathrm{HbAlc}$ values were found to have greater all-cause mortality risk. In addition, lower all-cause mortality risk was found among individuals with an $\mathrm{HbA} 1 \mathrm{c}$ of less than $6.5 \%$. Stratification by diabetic groups did not result in any statically significant relationships although the hazard ratios were in some cases in opposite directions. 


\section{CHAPTER V.}

\section{DISCUSSION}

\section{$\underline{\text { Overview }}$}

In diabetes management, although glycemic control is one of the main outcomes for determining treatment prognosis, it continues to be inadequate in the US diabetic population, with about half not having good control. ${ }^{27}$ Not being able to reach glycemic control, despite adherence to diabetes management recommendations, can lead to frustration, indifference, and unwillingness to continue treatment. ${ }^{135}$ In the present study we attempted to assess both risk factors for suboptimal, borderline, and poor glycemic control as well as its outcomes with the expectation of gaining knowledge of why there may continue to be a high prevalence of suboptimal and poor glycemic control among adults with diabetes despite medical awareness of their importance.

\section{$\underline{\text { Potentially new risk factors and glycemic control }}$}

There has been much research into the possible determinants of glycemic control. Most studies have repeatedly focused on demographic risk factors such as raceethnicity $^{37}$ as well as characteristics specific to diabetes such as duration of diabetes and type of diabetes treatment. ${ }^{35,136}$ From the review of the literature of suboptimal and poor glycemic control, it appears that other potential risk factors for suboptimal and poor glycemic control may exist. For example, although it is not surprising the lack of adherence to diabetes treatment is shown to be associated with poor glycemic control, ${ }^{39}$ reasons for this incompliance are not entirely known. Possible reasons for the incompliance and consequent poor glycemic control may include a diabetic's fear of 
weight gain. It may also be that there are barriers in the diabetic's environment preventing them from being compliant to diabetic treatment advice, such as lack of time to take medication or eat regularly because of inflexible work schedules. Furthermore, although an association between diabetes complications and glycemic control and smoking has been found, ${ }^{60-64}$ a factor closely associated, secondhand smoke exposure, has not been fully investigated.

\section{$\underline{\text { Glycemic control and work hours }}$}

Work constitutes an important part of most adult lives and sometimes is even a culprit of ill health. Long work hours has been implicated as a risk factor for poor health, including increased risk of mortality, ${ }^{137,} 138$ increased accidents, ${ }^{139}$ cardiovascular disease, ${ }^{140-142}$ greater unhealthy behaviors such as alcohol consumption, ${ }^{143}$ increased smoking, ${ }^{144}$ and less participation in physical activity. ${ }^{145}$ Thus, it is possible that glycemic control is also affected by having long hours of work.

Most research studies on the effects of long work hours on diabetes and glycemic control have been based on non-US populations. ${ }^{146,147}$ However, the consequences of long work hours should be of particular concern for American workers given that they report some of the highest number of work hours among industrialized countries. ${ }^{147}$ In fact, the amount of overtime in the US has increased since the 1970's. ${ }^{148,149}{ }^{147}$ Among 15 industrialized nations in the world, in 2003 the US ranked as the fourth highest in average annual work hours, preceded only by Thailand, Hong Kong, and South Korea. ${ }^{150}$ Meanwhile, the European Union does not allow more than 48 hours per work week, and Japan does not permit more than 100 hours of overtime per month. Yet in the US, only 
certain occupations (e.g. transport workers and medical residents) ${ }^{151}$ have restricted work hours by law, and there are regulations regarding the amount to be paid for overtime work but not limiting the number of overtime hours. ${ }^{152}$

In the present study we sought to understand the relationship between work hours and glycemic control. We found that among adults with type 2 diabetes who worked greater than 40 hours (about $34 \%$ of the sample) relative to those that worked 1-20 hours were at least 7 times as likely to have suboptimal or borderline glycemic control.

Although research has shown a relationship between work hours and cardiovascular disease, ${ }^{140-142}$ there is limited research specifically looking at its relationship with diabetes or glycemic control. Thus, the findings from the present study cannot be adequately compared to other studies. However, our findings are in agreement with studies in which individuals have reported irregular work hours and inflexible work schedule as a barrier to proper diabetes management. ${ }^{44,45}$

There are several reasons why working a greater number of hours may be associated with worse glycemic control. First, the reason for suboptimal glycemic control among those working long work hours may be simply related to the lack of time (i.e. time scarcity) to properly manage diabetes. For example, workers may not have enough time to check blood glucose levels or eat regularly or at scheduled time intervals. ${ }^{153,154}$ In a study in the Netherlands, a higher work load was perceived as a barrier to proper diabetes management, particularly insulin injection, in both type 1 and type 2 diabetes patients. ${ }^{153}$ Time scarcity has been associated with an increased risk of obesity and of making poor food choices. ${ }^{154}$ Weight gain and obesity, both risk factors for type 2 diabetes, have also been found to be greater among individuals working long hours. ${ }^{144}$ In addition, time 
scarcity is one of the barriers noted to be associated with the lack of adherence to a diabetic regimen or treatment. ${ }^{155}$ Furthermore, lack of time because of greater work hours may also interfere with important diabetes management activities outside of work such as getting medical care or visiting a diabetes healthcare provider or educator. For example, it has been shown that individuals who work a full-time or even a part-time job (as opposed to being unemployed or retired) are more likely to discontinue going to diabetes self-management education programs. ${ }^{156-158}$ In addition, lack of time has been associated with self-reported inability to visit dieticians. ${ }^{159,160}$

Second, longer work hours may result in suboptimal glycemic control due to greater job stress or strain as a result of working greater hours. Job strain has been linked to higher $\mathrm{HbA1c}$ levels among employed individuals without diabetes in non-US studies, ${ }^{42,46,47}$ while stress management programs have been associated with improved glycemic control. ${ }^{161}$ Although the biological mechanism is not well understood, stress may affect glycemic control via behaviors and neurohumoral pathways such as the counter-regulatory hormones, ${ }^{162,163}$ and glycemic control may be related to the allostatic load or body's way of adjusting to long-term stress. ${ }^{164}$ Poor glycemic control could also be in part due to the release of catecholamine and stress hormones (such as cortisol), which have been linked with increased cardiovascular risk factors. ${ }^{165,166}$ The elevated stress levels could also result in negative behavioral habits, such as increased eating, in order to cope with such stress. In fact, weight gain and obesity have been found to be the highest among individuals reporting high job strain. ${ }^{167}$

Third, it is possible that greater work hours may lead to worse glycemic control due to a greater propensity of late night eating since the time of day that meals are 
consumed has been reported to affect insulin levels and to increase glucose intolerance. ${ }^{168}$ Nevertheless, greater research is needed to understand the mechanism behind the relationship between long work hours and suboptimal glycemic control among adult with diabetes.

\section{$\underline{\text { Glycemic control and farm workers }}$}

With regards to our findings about farm workers being more likely to have suboptimal glycemic control compared to white collar workers, there are very few if any studies that have investigated the association between glycemic control and being a farm worker to compare to. There are several possible explanations to our findings nonetheless. For example, it may be possible that farm workers, compared to white collar workers, may have less knowledge about diabetes self-management, ${ }^{169}$ have inflexible work schedules, ${ }^{170}$ and be more likely to have unhealthy eating behaviors; ${ }^{171}$ all factors that may be responsible for poor glycemic control. For example, unhealthy diets are said to be greater among workers with high workloads, ${ }^{172}$ low status jobs, ${ }^{173}$ low control at work, ${ }^{174}$ and among workers with lower education and income ${ }^{175,176}$ all characteristics that may be more predominant among farm workers. Moreover, other factors such as social isolation, lack of social support, and depression, ${ }^{177}{ }^{178,179}$ among farm workers may explain their higher likelihood of poor glycemic control. For example, a study of farm workers with diabetes found that over $66 \%$ reported themselves as being depressed and were greatly concerned about the long-term consequences of diabetes. ${ }^{171}$ In addition, stress may be higher in farm workers compared to white collar workers and may be contributing to the worse glycemic control in this group. Farm workers may be stressed 
due to not knowing for how long they will be employed (fear of being unemployed), working strenuous hours, being away from the family, fear of being deported or discriminated against and exploited. ${ }^{171}$ This fear of losing their job may make the worker more hesitant to ask for time off and therefore be less able to access healthcare ${ }^{180}$ and routinely get diabetes care including getting their $\mathrm{HbAlc}$ checked and getting physician advice about diabetes. ${ }^{181}$

We could not assess the relationship between the above mentioned potential mediators of the relationship between work hours or farm worker occupation and glycemic control. This is because the data were not available in NHANES, except for the number of visits to a healthcare professional in the last 12 months. Nevertheless, the mediation test with that variable was not significant suggesting that the number of visits to a healthcare professional in the last 12 months does not explain the relationship between work hours or farm worker occupation and suboptimal/poor glycemic control.

\section{Glycemic control and desire to lose weight}

Research has shown that one of the barriers to adherence to diabetes treatment regimen is the fear of weight gain commonly associated with treatment regimens such as taking insulin. ${ }^{66-72}$ Furthermore, adolescents with diabetes perceiving themselves as overweight have reported poor glycemic control. ${ }^{73}$ Thus, it may be that individuals that want to lose weight are less likely to be adherent to treatment regimens and consequently have suboptimal or poor glycemic control. However, the association between glycemic control and weight desirability among adults with diabetes has been understudied. 
In the present study, a statistically significant association between glycemic control and desire to lose weight was not found. This finding is in agreement with a study among adolescents with both type 1 and type 2 diabetes that found that trying to lose weight or worrying about one's weight was not associated with poor glycemic control, although perception of being overweight was. ${ }^{73}$ Nevertheless, the null finding could also be a reflection of the two possible directions of the association between glycemic control and weight desirability. As the literature review indicated and as hypothesized, it is possible that individuals who wanted to lose weight and who knew of the possible consequences of tighter glycemic control such as weight gain, ${ }^{182-186}$ would be less likely to have suboptimal or poor glycemic control because of the lack of adherence to treatment. A typical recommendation in diabetes management is weight loss if the person is overweight or obese. ${ }^{187}$ Thus, it is also possible that those individuals who wanted to lose weight would adhere more to diabetes management advice and would therefore be more likely to have good glycemic control. Thus, it is possible that the null finding in the present study is a factor of having two types of individuals, those not adherent to diabetes management treatment due to fear of weight gain, and those adherent to diabetes management treatment due to wanting to lose weight and already understanding the detrimental effects of being overweight on diabetes prognosis.

There may also be other factors into play in the relationship between desire to lose weight and glycemic control. For example, among adolescents, research has shown that unhealthy weight loss practices is associated with poor glycemic control among females but not among males. ${ }^{73}$ Given the potential differences in the relationship between glycemic control and dieting behavior by sex, we tested the interaction between desire to 
lose weight and sex. We also conducted sex-specific analyses but did not find any statistically significant results. Therefore, more research is needed to understand the association between desire to lose weight and glycemic control.

\section{$\underline{\text { Glycemic control and secondhand smoke exposure }}$}

Studies have shown a relationship between smoking and glycemic control ${ }^{63,64}$ as well as diabetes complications that are often a result of poor glycemic control. ${ }^{61,62}$ Research on the various effects of SHS exposure on health outcomes aside from respiratory function is also mounting. Studies have recently documented the potential effects of SHS on cardiovascular disease, ${ }^{188,189}$ glucose intolerance and diabetes incidence. $^{43,65,190-192}$ Given the literature, it is plausible that SHS affects glycemic control as well.

We assessed the association between glycemic control and SHS among adults with type 2 diabetes but did not find a statistically significant relationship. Our results cannot be adequately compared to other studies since there are very few if any studies assessing the relationship between glycemic control and SHS exposure. Nevertheless, the lack of statistical significance may also be a result of lack of power. It may also be that our definition of SHS exposure did not properly capture individuals with SHS exposure due to the short half-life of detectable serum cotinine levels, ${ }^{193}$ not knowing how long individuals were exposed to secondhand smoke, the absence of self-reported exposure to smoke in other settings (e.g., restaurants, bars, or motor vehicles), and potential misclassification of smoking and SHS exposure status. ${ }^{124}$ Because of this potential misclassification, analyses were also done using cotinine as a continuous variable among 
individuals who reported not being current smokers but no statistically significant relationship was found with this measure of SHS either. Therefore, because of the possibility of error in defining smoking and SHS exposure groups, the uncertainty of the accuracy of the measure for SHS exposure (which may explain the lack of statistical significance), and because other studies have suggested that SHS effects glycemic control, ${ }^{42,}{ }^{64}$ further research is needed to fully understand the relationship between SHS exposure and glycemic control among US adults with type 2 diabetes.

\section{Glycemic control and health related quality of life}

Quality of life measures have been gaining interest in the medical and public health professions over the past years. In the late 1960's to early 1970's, HRQOL was mentioned approximately 40 times in the medical literature while in the late 1980 's to early 1990's it was mentioned approximately 10,000 times. ${ }^{194}$

The HRQOL measures may indicate how an individual views his or her health. This self-reflection of health status has been suggested to be a better indicator or ill health and good predictor of mortality. ${ }^{195}$ The determinants of how a person's self-perceived health may be based on an array of factors such as family history and other risk factors, medical history, and psychological orientation (i.e., optimism or pessimism). ${ }^{195}$ Furthermore, based on the premise of the "self-regulation theory", one's perception about the seriousness of one's illness does affect how one's disease is managed. ${ }^{196}$

How HbAlc affects everyday activities may be important given that individuals may perform certain self-management tasks as a result of short-term benefits such as lack of negative symptoms that may accompany poor glycemic control. ${ }^{77,} 197$ Social activities 
such as gatherings with friends are among the activities that may be affected by poor glycemic control because of fear of embarrassment from having the manifestation of hypoglycemia or hyperglycemia symptoms in public. An individual may be more likely to adhere to diabetes treatment if she or he knows that by following their physician's advice about proper diet for glycemic control, their respective risk of hypoglycemia or hyperglycemia may be lowered. In fact, among adults with diabetes, knowledge of HbA1c levels has been noted as an important factor determining how a patient rates his or her health, particularly physical functioning, mobility, and satisfaction with physical health and family life. ${ }^{198}$ In addition, among adults with diabetes, barriers for lack of compliance have been noted to include lack of symptoms ${ }^{155}$ and the belief that having diabetes is not serious. ${ }^{199}$ Patients with diabetes have stated that managing their diabetes is associated with an impaired ability to engage in activities with their family and friends. ${ }^{200}$ Thus, bringing awareness of how poor glycemic control affects everyday life may lead to better treatment compliance.

Diabetes is a disease that requires self-management behaviors to improve treatment prognosis. The adherence to treatment and the positive self-management behaviors may be influenced by symptoms and physiologic changes due to the diabetes disease, which may take years develop or be physiological manifested. ${ }^{200}$ Thus, understanding the impact of good management practices on day-to-day activities and well-being may lead to better adherence and ultimately improved glycemic control.

There have been studies assessing the relationship between glycemic control and quality of life, but these have been contradictory and/or are not based on nationallyrepresentative US population. ${ }^{83-88,201,202}$ Thus, we attempted to assess the relationship 
between glycemic control and HRQOL. We found that these relationships were not the same for all HRQOL indicators. Specifically, we observed a statistically significant relationship between poor glycemic control and number of days with poor mental health and inactivity. However, poor glycemic control was not associated with number of days of poor physical health or poor self-reported general health.

Our findings are in agreement with the literature which has shown that suboptimal and/or poor glycemic control is not related to all aspects of health in the same fashion. ${ }^{83-}$ ${ }^{88}$ These studies have however defined quality of life based on SF-36 scores, the validated instrument with 36 questions about perceived health status and number of disability days. This instrument is considered the gold standard for evaluating overall health including physical and mental health. ${ }^{78}$

For example, one study of patients from community health clinics in California followed for one year showed a slight inverse relationship (-0.21 regression) between HbA1c levels and SF-36 Mental composite scores (higher score indicating better mental health). ${ }^{83}$ However, in that same study ${ }^{83}$ no significant relationship was observed with the SF-36 Physical Composite Score. In another study of diabetic adult patients from a Veterans Affairs Health Care system, perceived poor health (as indicated by SF-36 scores) was associated higher $\mathrm{HbAlc}$ levels but mental health was not associated. ${ }^{85}$ Other studies have not found any significant relationship between overall quality of life and glycemic control. ${ }^{81,201,202}$ For example, in a study of non-insulin diabetic patients of a Veteran's clinic in North Carolina, HbA1c levels and HRQOL assessed using SF-36 were not correlated. ${ }^{202}$ 
Glycemic control and self-reported general health, physical health, and activity limitation

A statistically significant relationship between self-reported general health and glycemic control was not found in our study, which is not surprising given the inconsistent findings in previous studies. For example, in a double-blind randomized controlled trial of adults with type 2 diabetes, the treatment group with more strict diabetes treatment regimen (aimed at improving glycemic control) reported better selfperceived general health and improved cognitive functioning. ${ }^{102}$ However, other studies have not found a statistically significant relationship between self-reported general health and quality of life. ${ }^{88}$

We also did not observe a statistically significant relationship between glycemic control and number of days of poor physical health. This is in contradiction to research that has suggested poor glycemic control to be associated with poor physical health given the greater likelihood of diabetes complications that cause chronic pain such as neuropathy among diabetic adults with poor glycemic control. ${ }^{194}$ Nevertheless, we cannot compare our findings to other studies due to the lack of studies specifically investigating the relationship between glycemic control and number of days of poor physical health.

We did, however, find a statistically significant relationship between days of limited activity and glycemic control. Specifically, those with poor glycemic control were more likely to have $\geq 14$ days of inactivity (versus 0 days) but were less likely to have 113 days (versus 0 days). These findings are inconsistent with the literature on disability and diabetes and glycemic control. ${ }^{94,100}$ For example, a study using data from the Midlife Development in the United States Survey among adults between the ages of 25 and 54 years, noted diabetics to have an average of 3.6 impairment days per month although 
diabetes ranked lower than other conditions such as cancer, ulcer, heart disease, and generalized anxiety disorder in terms of the number of impairment days per month. ${ }^{94}$ Studies specifically investigating the relationship between self-reported activity limitation and glycemic control were not found however.

A potential reason for the lack of statistically significant associations between days of poor physical health and days of inactivity and glycemic control could be due to the categorization of these variables, as these are somewhat arbitrary categories used in previous studies. ${ }^{115,116}$ The application of categories for these variables could have also accounted for the differences in the directions of the estimates for days of poor mental health. Since the numerical variables of number of days of poor physical health, number of days of poor mental health, and days of inactivity showed over-dispersion and excess zeros, ZIP analyses were conducted as opposed to traditional linear regression analyses. The ZIP analyses showed no statistically significant relationships between number of days of poor mental or physical health or inactivity with glycemic control. Therefore, further research may be needed, with perhaps better measures, to establish is there is a relationship between number of days of poor health and inactivity and glycemic control among US adults with type 2 diabetes.

\section{Glycemic control and mental health}

In the present study suboptimal and borderline glycemic control was associated with lower odds for having 1-13 days of poor mental health (versus 0 days). These findings suggest that individuals with suboptimal glycemic control rather than good glycemic control have better mental health. Thus, there may be a level of distress or 
mental discomfort associated with having to maintain very low HbA1c levels among adults with type 2 diabetes. Our findings are in agreement with studies that have found that tight or strict glycemic control (e.g. HbAlc levels of less than or equal to $6.5 \%$ ) is associated with poor quality of life measures of mental health and physical health. ${ }^{198,203 \text {, }}$ ${ }^{204}$ One of the reasons why those individuals with tight glycemic control rate their health as poorer may be due to greater episodes of hypoglycemia when $\mathrm{HbA} 1 \mathrm{c}$ levels are lower, ${ }^{198,205,206}$ which can range from 2 to 4 episodes per year among those type 2 diabetics trying to manage their diabetes. For example, the Diabetes Control and Complications Trial (DCCT) study showed that strict glycemic control increases the chances of severe hypoglycemia. ${ }^{206}$ Hypoglycemia may cause psychological symptoms such as displeasure, feelings of anger, and fatigue ${ }^{207}$ as well as more visible physiologic symptoms such as poor coordination, nausea, drowsiness, shaking and sweating. ${ }^{208,209}$ Researchers have also suggested a curvilinear relationship between $\mathrm{HbA1c}$ levels and quality of life likely due to the negative aspects of either the intricate treatment regimen and/or the greater episodes of hypoglycemia due to tight glycemic control. ${ }^{79}$

In addition to the symptoms of hypoglycemia, the greater days of reported poor mental health may be due to the sometimes overwhelming chore of diabetes selfmanagement. The diabetes regimen can be mentally taxing since it may require drastic dietary changes, and the re-scheduling of activities (due to, for example, having to take medications or insulin injections at certain times of the day or having to eat regularly). ${ }^{39}$ Furthermore, these lifestyle changes in everyday activities may make others annoyed which may then result in conflict between the individual with diabetes and his/her friends, family and co-workers. This conflict may be a contributor to the mental stress 
that adults with diabetes often report. ${ }^{194}$ Finally, the fear of hypoglycemic events due to such strict glycemic control, particularly in social settings, may place an additional level of emotional distress to the individual with diabetes. ${ }^{194}$ There are no known studies however investigating the relationship between fear of hypoglycemia and glycemic control among adults with type 2 diabetes. As hypothesized however, poor glycemic control (i.e. HbAlc $\geq 9$ ) was found to be associated with having greater days of poor mental health. There are several potential explanations for our findings. One is that the negative symptoms associated with hyperglycemia (i.e. increased thirst, urination, and weight loss, as well as fatigue, drowsiness, depression) ${ }^{210}$ are higher when the person has their blood glucose levels out of control, thereby leading to reduced feelings of wellbeing and consequently report of poor mental heath. ${ }^{80,211}$

On the other hand, it may be that these individuals with diabetes have poor glycemic control because they are depressed and are in poor mental health to begin with. This scenario is very likely given the high prevalence of depression among adults with diabetes, with data suggesting that as many as $9-27 \%$ of the diabetes population may experience depression. ${ }^{212,213}$ Thus, it is possible that depression preceded poor glycemic control in our study sample. These individuals with depression may be having poor glycemic control due to being more likely to be non-adherent to treatment regimens. ${ }^{214}$ Individuals with poor mental health may be in denial of their condition and therefore not follow diabetes management steps thereby leading to further poor glycemic control. ${ }^{215} \mathrm{In}$ addition, for a percentage of hard to treat individuals with type 2 diabetes, particularly those newly diagnosed, the lack of reaching good glycemic control levels despite taking measures may lead to feelings of disappointment despair, emotional distress, and 
consequently poor self-reported mental health. ${ }^{39,194,216}$ Thus there may be a "negative reinforcing cycle" in which lack of attainment of good glycemic control despite good efforts leads to emotional distress, and the emotional distress leads to lack of encouragement and hope and consequently lack of adherence to treatment, which further leads to even worse glycemic control. ${ }^{39}$ Finally, there may be biological mechanisms for the relationship between depression and glycemic control. For, example it has been suggested that decreases in serotonin and cortisol due to depression may increase fasting glucose levels. ${ }^{217}$ More research is need in this area however.

Although we did not find a statistically significant interaction between sex and number of days with poor mental health, to be consistent with the literature suggesting a relationship ${ }^{218,219},{ }^{220} 221$ we conducted sex-specific analyses for the relationship between poor glycemic control and poor mental health. In sex-specific analyses, we found that among females with type 2 diabetes, poor glycemic control was associated with lower odds for having 1-13 days (versus 0 days) of poor mental health while among males, poor glycemic control was associated with greater odds of 1-13 days (versus 0 days) of poor mental health. Our findings are in agreement with studies that have shown that among women, poor glycemic control is associated with poor overall quality of life (i.e., not specifically mental or physical health). ${ }^{218,219}$ Furthermore, in a Netherlands study, there was an observed positive association between depression and poor glycemic control among women but not among men. ${ }^{220}$ This association may be greater among women because of the lower estrogen levels as women become older. ${ }^{221}$ In fact, a study found that estrogen replacement therapy was associated with improvement in glycemic control. $^{221}$ In addition, depression has been associated with changes in estrogen levels. ${ }^{222}$ 
However, one study found depression to be associated with higher HbA1c levels in men but not in women. ${ }^{223}$ Thus, the contradictory study findings suggests that further research is needed to truly understand the sex differences in the relationship between depression and poor mental health and glycemic control.

Finally, to further investigate why there was a difference in the direction of the estimate for the relationship between poor mental heath and glycemic control, multinomial logistic regression analyses for poor mental heath were repeated using collapsing zero to 5 days of poor mental health into one category. The results, however, did not reveal any difference whether having zero days as one category by itself or grouping it with 1-5 days.

\section{Workforce participation and glycemic control}

In addition to morbidity outcomes and HRQOL including self report of wellbeing, workforce participation is another measure of the effect of ill health and the overall burden of disease. Two commonly used measures of workforce participation are employment status and missed work or absenteeism. Research has shown that adults with diabetes are less likely to be employed and more likely to miss work, ${ }^{57,97-99,201,224-226}$ particularly if they have complications from diabetes. ${ }^{94,100,101,227-229}$ This may be due to diabetes being a health condition unlike many others that requires self-management in order to improve disease prognosis, reduce the risk of complications, and increase overall quality of life. ${ }^{230}$ Individuals with diabetes usually need to follow strict dietary regimens as well as medication regimens that require medication (such as insulin injections) to be followed in a scheduled manner. These regimens may thus need to be implemented 
during work hours, which may appear burdensome to both the employee and the employer. Such frustration and inability or unwillingness to properly accommodate the employee may lead to unemployment. In turn, the lack of proper diabetes management and consequently poor glycemic control and related symptoms may result in greater absenteeism from work.

Since poor glycemic control is a factor that greatly increases the chance of diabetes complications, it is more likely that glycemic control is associated with unemployment and missed work. In the present study the relationship between employment status, missed work days, and glycemic control was assessed.

\section{Glycemic control and missed work}

In the present study, a statistically significant relationship between glycemic control and missed work days in the previous month was not found. The null finding in the present study could be due to two reasons.

First, there may not be a relationship between glycemic control and missed work, since there are contradictory findings in the literature. Similar to the present study, some studies have not found a statistically significant relationship. For example, in a crosssectional study of adults with diabetes referred to a diabetes program (the Control Diabetes Services Program) from several centers in the US, there was marginally statistically association between an $\mathrm{HbA} 1 \mathrm{c}$ level of $\geq 8 \%$ and self-reported days lost from work or school during the previous year, after controlling for confounders. ${ }^{104}$ However, in that study, ${ }^{104}$ an $\mathrm{HbA} 1 \mathrm{c}$ of $8-10 \%$ was not statistically associated with days lost from work or school during the previous year. Furthermore, HbAlc levels of 7-8\%, HbAlc 8- 
$10 \%$, or $\mathrm{HbAl} \mathrm{c}$ of $\geq 10 \%$ was associated with having greater than five days of missed work in the previous month or with difficultly accomplishing work tasks among adults with diabetes enrolled in health maintenance organization in the US. ${ }^{227}$ However, other studies have found a statistically significant relationship. In a cross-sectional of patients with diabetes from Michigan, having an $\mathrm{HbAlc}$ of $8-8.9 \%$ was associated with greater probability of missing at least one hour of work using probit models. However, a statistically significant relationship was not found for HbAlc levels of 7.0-7.99\% or greater than $9.0 \% .{ }^{103}$ Furthermore, in that study,${ }^{103}$ missed work was based on hours missed from work in only the last 4 weeks, which may not be accurate estimation of the habitual missed work due to glycemic control issues.

Second, literature on absenteeism related to health, or sickness absence, has suggested that absenteeism is not a factor that is solely determined by the presence of a medical condition, but rather based on different factors such as an individual's perception of his/her health and symptoms related to disease, attitudes related to work and missing work, job demands and accommodations at work, job strain, and other factors. ${ }^{231}$ Thus, it is possible for example that some of these individuals with diabetes are resistant to missing work regardless of how ill they feel from being in poor glycemic control. ${ }^{232}$

\section{Glycemic control and unemployment}

In the present study we did not observe a statistically significant relationship between glycemic control and employment status. Our findings are in disagreement with the sparse studies assessing the relationship between glycemic control and employment status. In a study of health maintenance organization enrollees with diabetes, HbAlc 
levels of $\geq 10 \%$ were associated with greater disability and unemployment. ${ }^{227}$

Furthermore, in a double-blind randomized controlled trial of adults with type 2 diabetes the treatment group with more restrictive diabetes treatment regimen were less likely to report losing their jobs during the follow-up period. ${ }^{102}$

There are several potential reasons for the lack of a statistically significant relationship between employment and glycemic control. First, there is the potential of misclassification of employment given that employment is based on working in the prior week. Thus the person would be considered unemployed when in fact they were employed. Second, it is possible that unemployment (or employment) is a result of other factors not related to health. For example adults 65 years of age or older may be more likely to unemployed because of retirement and not necessarily due to their level of glycemic control. However, when analyses were repeated removing those 65 years and older, there were no statistically significant relationships between glycemic control and employment status in those less than 65 years of age or 65 years of age or older. Third and lastly, there may be the potential for healthy worker effect bias. If healthy worker effect bias is present, then the true association between employment and glycemic control would be masked given that those employed are generally in better health than those not employed.

Although no statistically significant interactions were found, since the literature suggests that there may be differences in employment due to sex ${ }^{129}$, sex-specific analyses were conducted. When the full model was stratified by sex, the results remained nonstatistically significant but the direction of the association was different for males and females. 


\section{Mortality and glycemic control}

It is well established that individuals with diabetes are at an increased risk of death compared to individuals without. The mortality rate ratio of the mortality rates comparing individuals with diabetes versus those without has been shown to range from 1.5 among individuals age 65-74 years of age to 3.6 among individuals $25-44$ years of age. ${ }^{233}$ Not surprisingly, diabetes is the sixth leading cause of death based on the most current national death statistics with a death rate of 23.3 per 100,000 US population according to CDC 2006 data. ${ }^{6,234}$ One of the proposed reasons for the higher mortality risk is poor glycemic control among individuals with diabetes since poor glycemic control is associated with increased diabetes complications. ${ }^{10}$

Several studies have assessed the relationship between glycemic control and allcause and cause-specific mortality risk, although these have led to conflicting findings, particularly when analyses were sex-specific analyses, and/or based on non nationally representative sample of adults with diabetes, specifically type 2 diabetes. ${ }^{105-107}$ The only nationally representative study assessing glycemic control among adults with type 2 diabetes that was recently published found that there was a curvilinear relationship between HbA1c levels and all-cause and CVD mortality risk. ${ }^{235}$ However, that study ${ }^{235}$ did not assess the relationship between mortality risk glycemic control among diabetic groups such as those that may be in presumably worse health (e.g. older, insulin taking type 2 diabetics, or those with hypertension) and therefore be at greater risk of complications from very strict glycemic control. In addition, that study ${ }^{235}$ did not address the potential impact of confounding by depression in the relationship between glycemic control and mortality risk. Assessing the role of depression in mortality risk is important 
given it has been associated with increased mortality risk among adults with diabetes. ${ }^{236}$ In addition, depression has been associated with poor glycemic control, ${ }^{214,223}$ plus we found poor glycemic control to be associated with number of days of poor mental health.

In this study we assessed the effect of suboptimal, borderline, and poor glycemic control on all-cause and CVD mortality risk. We found that neither suboptimal, borderline, or poor glycemic control was significantly associated with increased all-cause or CVD mortality risk, after adjusting for several potential confounders. Nevertheless, the results did show an effect in the hypothesized direction, with higher non-statistically significant mortality hazards due to suboptimal or poor glycemic control.

Since the literature suggests that there are differences in mortality by $\operatorname{sex}^{130}$ and because there was an interaction between BMI category and sex, sex-specific adjusted hazards models were also undertaken. Even in sex-specific analyses, non-statistically significant relationships were found between the mortality risk and the dichotomous variables for glycemic control.

In order to be consistent with the studies on mortality risk and glycemic control, we also used the HbA1c measure as a continuous variable, ${ }^{106-108}$ but no statistically significant relationship was found, except for when stratifying the results by sex, with statistically significant results found for males only. This finding suggest that perhaps the benefits in life expectancy due to improved glycemic control may be more pronounced in males rather than females, however more research in this area is needed.

We did not find that depression was associated with any of the glycemic control groups nor was it associated with increased mortality risk. Therefore, it was not believed to be a potential confounder in the relationship between glycemic control and all-cause 
mortality risk and thus was not added in the final hazard models. Indeed, adding depression in the hazard models did not result in any change in the association between all-cause mortality and glycemic control.

Although we hypothesized that suboptimal, borderline, and poor glycemic control would be associated with greater odds of mortality, the findings are not surprising given the contradictory findings in previous studies. Among studies that have looked at allcause mortality only, a statistically significant relationship with inadequate glycemic control has been observed. For example, poor glycemic control has been associated with all-cause mortality among advanced chronic kidney disease (CKD) and dialysis patients. ${ }^{105}$ In a study of patients undergoing maintenance hemodialysis, greater HbA1c values predicted a greater risk of all-cause and cardiovascular mortality among adults with diabetes after three years of follow-up, after adjusting for confounders such as demographics, malnutrition and anemia. ${ }^{107}$ In a prospective study of individuals primarily without diabetes in the United Kingdom to assess the relationship between HbAlc values and all-cause mortality and CVD mortality after a 6 year follow-up, ${ }^{108}$ it was shown for every $1 \%$ increase in HbAlc level, the all-cause and CVD mortality risk increased in a dose response fashion. However this study ${ }^{108}$ did not control for family history of diabetes or CVD and other potential confounders.

One study, however, did not find a statistically significant relationship between glycemic control and both all-cause mortality or CVD mortality risk. ${ }^{106}$ In a study using data from the Modification of Diet and Renal Disease (MDRD) study, a randomized controlled study of non-diabetics with $\mathrm{CKD}$, a $1 \%$ increase in $\mathrm{HbAlc}$ was associated with a greater risk of all cause mortality, but the association with CVD mortality was not 
significant, after approximately a 7-11 year follow-up period. ${ }^{106}$ However, in this study, the incidence of diabetes was not established, a factor which could have distorted the results. Moreover, most of the studies investigating the relationship between diabetes management measures (e.g., fasting blood glucose, HbAlc value) and CVD events including mortality risk has been primarily in men. ${ }^{108-110,237}$

In addition to studies on the effect of suboptimal and poor glycemic control on mortality risk, there has been recent attention to the effects of more strict thresholds for glycemic control on mortality. ${ }^{238-240}$ Strict glycemic control is generally considered an $\mathrm{HbAlc}$ value of less than or equal to $6.5 \%$ and is often reached by intensive diabetes management, for example with treatment with sulfonylurea or insulin and with the goal of reducing their fasting plasma glucose concentration to less than $108 \mathrm{mg} / \mathrm{dl}$. Such intensive treatment has been associated with reductions in the incidence of nephropathy, neuropathy, and retinopathy complications by as much as $27 \%{ }^{241}$ and has been shown to lead to increases in life expectancy, with a slight increase in complications due to longer survival time. ${ }^{241}$ However, negative health outcomes due to strict glycemic control, such as increased mortality risk, have also been reported. For example, in the Action to Control Cardiovascular Risk in Diabetes (ACCORD) study, individuals in the strict glycemic control arm, who achieved on average an $\mathrm{HbAlc}$ of $6.4 \%$, had greater deaths $(n=257)$ compared to the group not in the strict glycemic control (with average HbAlc of 7.0-7.95) ( $\mathrm{n}=203$ deaths) event after four years of follow-up. ${ }^{242}$ This translates into a $1.4 \%$ death rate per year for the strict glycemic control arm versus $1.1 \%$ death rate per year for the control group. ${ }^{242}$ This unexpected result in the ACCORD study resulted in termination of the trial and suggested that very strict glycemic control among certain 
individuals with type 2 diabetes is harmful to health. ${ }^{242}$ However, the findings of this trial should be interpreted with caution since the participants were perhaps more representative of the diabetics with worse health given that most had elevated HbA1c (average of 8.2 at baseline), had greater length of diabetes duration, were smokers, had high cholesterol and high blood pressure and were obese; these factors could have increased the mortality risk. ${ }^{242}$ Nevertheless, continued debate exists as to whether strict glycemic control is beneficial in terms of decreasing mortality risk to all individuals, or whether for some more chronically-ill individuals, intensive treatment can cause more harm than good.

Given the recent controversy and remaining questions as to which threshold of glycemic control is most beneficial for improving overall health and reducing mortality risk, ${ }^{238-240}$ we performed additional analyses using stricter thresholds for glycemic control. We found that indeed having an $\mathrm{HbAlc}$ value of less than $6.5 \%$ was protective against all-cause mortality among adults with type 2 diabetes. These results are in agreement with studies among hemodialysis patients. ${ }^{107}$

The literature also suggests that strict glycemic control may be particularly harmful to certain diabetic groups such as the older (and therefore likely more frail), those taking insulin only, and/or those with comorbid conditions such as hypertension, as implied by results of the ACCORD study. ${ }^{242}$ However, analyses conducted stratifying by diabetic groups did not show statistically significant differences associations between mortality risk and glycemic control, although the hazard ratios were sometimes in the opposite directions, suggesting possible moderation. Given the differences in direction of the relationship between all-cause mortality risk and strict glycemic control, the results 
suggest that the effect of strict glycemic control on mortality risk may depend on the age, gravity of diabetes condition (i.e. whether dependent on insulin or not), and the presence of other comorbid conditions. Greater research is needed in this area however since these results were not statistically significant.

Thus, our findings suggest that strict glycemic control may indeed be beneficial in increasing survival among adults with type 2 diabetes after a 6 to 12 year follow-up, assuming glycemic control remained relatively constant through the follow-up period. These findings would be therefore in disagreement with recent findings from the ACCORD study. ${ }^{242}$ Differences in diabetic populations in the ACCORD study and the present study could account for the different findings related to mortality risk from strict glycemic control.

\section{$\underline{\text { Strengths and limitations }}$}

This study has both strengths and limitations. A limitation of this study was its cross-sectional design, which does not allow for the establishment of causal relationships. However, the information gained from this research can generate hypotheses that could be tested in future analytical studies using for example cohort and prospective designs.

In addition, the individual's diabetes status and duration of diabetes were based on self-reported information. However, self report of diabetes has been found to be fairly accurate when compared to medical criteria, with overall agreement of $96.3 \%$ (sensitivity of $85.2 \%$ and specificity of $98.3 \%) .{ }^{243}$ In addition, whether the individual had type 1 or type 2 diabetes is not truly known as individuals were only asked about diagnosis of diabetes in general not whether their diabetes was type 1 or type 2 . The definition for 
type 2 diabetes that was used in the present study was modeled after definitions used in several studies in which diagnosis of diabetes after the age of 30 was considered type 2 diabetes. ${ }^{30,244,245}$ However, since type 2 diabetes has been shown to be increasingly diagnosed in much younger age groups, ${ }^{246}$ there is the potential for misclassification with the definition that was used that assumes that individuals diagnosed before the age of 30 have type 1 diabetes.

Also, glycemic control was based on only one $\mathrm{HbAlc}$ reading leaving the possibility of measurement error of glycemic control. However, the possibility of measurement error is minimal given that the NCHS HbAlc laboratory protocol includes strict quality control procedures to limit measurement error. ${ }^{114}$ Specifically, a subset of laboratory specimens of $\mathrm{HbA} 1 \mathrm{c}$ are randomly selected and replicated to test the validity of the measurements. The coefficient of variation of these specimens of range from $0.90-$ $2.54 \%,{ }^{114}$ thereby showing high accuracy of the measurements retested. In addition, research using NHANES III data has shown low variability and high sensitivity of HbAlc measurements repeated after a two week period in an subset of individuals from NHANES, with a within-person coefficient of variation of $3.6 \%{ }^{247}$ Thus, not only do the HbA1c measurements in NHANES data appear to be relatively free of measurement error due to for example faulty equipment or laboratory procedures. In addition, an individual's level of glycemic control as determined from $\mathrm{HbA} 1 \mathrm{c}$ tests do not appear to vary much even if taken within a few weeks apart. Nonetheless, given the cross-sectional design of the study and that a diabetic's degree of glycemic control may vary from a time point to another (e.g. year to year) and that the consequences of unhealthy lifestyles or environments (e.g. arduous working conditions) may take months or years to be 
manifested physiologically, the exact nature of the relationship between glycemic control, work characteristics and mental health may not be ascertained from this study.

Furthermore, although the likelihood of these to alter results are minimal, there may be some factors affecting the HbA1c reading that could lead to erroneous results such as hemoglobin disorders, erythrocyte mass and plasma volume, renal disorders, liver or cardiac function problems, anemia, and menstruation. ${ }^{248,249}$ There is also the possibility of recall-bias, for example for hours of missed work in the past 12 months and self-report of age of diagnosis, although studies have shown that age of diagnosis of some diseases is reliable. ${ }^{250}$

Furthermore, our findings may be skewed due to the healthy worker effect bias, which says that individuals tend to be in better health than non-employed individuals, or those working more hours are in better health than those putting fewer hours at work. If such bias is present in this study, then our results would be attenuated and the association between suboptimal/poor glycemic control and long work hours is even stronger than what the study showed. In other words, one would expect those working 1-20 hours to be in worse glycemic control than those working greater than 20 hours, yet we found the opposite relationship. In addition, we did not find a statistically significant association between employment status and glycemic control. Thus, a healthy worker effect bias is somewhat unlikely in the present study. Of note, only the analyses for hypothesis 1 and $2 \mathrm{~b}$ using the work variables were based on the employed sample. The analyses for assessing the relationship between glycemic control and SHS, weight desirability, HRQOL, and mortality risk were done using both employed and unemployed individuals, with most analyses controlling for employment status. 
Another limitation in the present study is that other possible determinants of glycemic control were not measured, such as patient adherence to medication dietary regimen, ${ }^{251,252}$ frequency of self blood glucose monitoring, ${ }^{253}$ health literacy, ${ }^{254,255}$ diet 256, 257 and frequency of meals consumed per day and caloric beverages. ${ }^{45}$ Psychological factors that may also be related to glycemic control were not assessed because these were not available in NHANES. These factors include stress, ${ }^{258}$ depression, ${ }^{214}$ beliefs regarding how serious the disease is. ${ }^{259}$ It would have also been beneficial to know the length of diabetes management or treatment. The length of time the individuals have had either good or poor glycemic control is also not known. Yet, the length of time of glycemic control is important given that the risk of diabetic complications, morbidity and mortality may be dependent on this. ${ }^{105}$ For example, in the DCCT study, renal improvements were evident after three years of good glycemic control. ${ }^{206}$ In addition, the degree of hypoglycemic events in the individuals is not known, although hypoglycemia and fear of hypoglycemia is a barrier to good glycemic control, ${ }^{208,260,261}$ particularly among those treated with insulin. ${ }^{262}$ We may have also an unreliable measure of missed work in the present study. This absenteeism measure is based on self report of missed work in the past month, which may be subject to some recall bias. In addition, ideally missed work would be based on the missed work in the last three months, given that glycemic control is based in average glucose levels in the last three months. We also assume that missed work is directly due to poor glycemic control when in fact it could be related to other factors not related to health. For example, sickness absence has been reported to be due to a combination of personality factors such as "coping mechanism" ${ }^{231}$ and "behavior of social inequity," ${ }^{263}$ and burnout. ${ }^{264}$ In addition, the use of ZIP 
analyses could have possibly not captured the association between missed work days and glycemic control. However, categorization of the variable missed work days (as opposed to leaving it as a count variable) did not result in any statistically significant relationship between it and glycemic control groups either. All of these factors could have led to the null finding when assessing the relationship between missed work and glycemic control.

We also do not have data on depression for NHANES 1999-2004, yet it may partially explain the relationship between employment and glycemic control since depression has been linked with poor glycemic control ${ }^{214}$ and with unemployment and disability. ${ }^{227}$ In addition, reduced performance while at work due to ill health, in other words presenteeism, was not measured although it can be an additional indicator of the negative effects of poor glycemic control.

Furthermore, the NHANES utilized a generic instrument to measure quality of life that was used instead of a disease specific one, which can result in reduced sensitivity. ${ }^{81}$ Although CDC's measure for HRQOL is a generic measure, therefore having the benefits of being able to be compared to findings from people living with other conditions, ${ }^{77,79}$ it might not be the most appropriate measure for diabetics. HRQOL scales that are more "illness-oriented" for diabetics include the Diabetes Quality of life Measure, the Diabetes Treatment Satisfaction Questionnaire, and the Problem Areas in Diabetes Questionnaire. ${ }^{77,79}$ These measures are better in that they can assess the impact of activities or experiences specific to diabetics, such as issues related to using pharmacological medication and diet. ${ }^{79}$

Another limitation is that mortality risk is only based on a 6-12 year follow up since NHANES III data was collected from 1988 to 1994 but the mortality linkage data 
are up to 2000. However the psychological and physiologic effects of poor glycemic control may take longer to be manifested. There may also be some degree of misclassification of cause of death. ${ }^{265}$ For example, a study comparing death certificates for coding of coronary heart disease as the underlying cause of death with cause of death judged by a panel of physicians showed that these death certificates have a sensitivity of $83.8 \%$, with a $24.3 \%$ overrepresentation of coronary heart disease deaths. ${ }^{265}$

Furthermore, level of glycemic control was only assessed at baseline in NHANES III and therefore level of glycemic control at time of death is not known, leading to potential misclassification. Finally, the small sample size of farm workers likely resulted in loss of precision of the estimates; this was evident in the widening of the confidence intervals as covariates were added in the regression models.

Despite these limitations, the present study has many strengths. The strengths of the present study included the use of data from a nationally representative sample of adults with type 2 diabetes, the availability of several potential confounders, the availability of a 6 to 12 year mortality follow-up of a nationally representative sample of adult with diabetes, the first known study to address the association between health related quality of life, workforce participation and glycemic control while adjusting for potential confounders, and the first study to assess the relationship between glycemic control and weight desirability, secondhand smoke, and work hours and type of occupation among US adults with diabetes. 


\section{CHAPTER VI.}

\section{CONCLUSION}

The aims of the present study were to assess the relationship between four potentially new risk factors (number of hours worked per week, type of occupation, secondhand smoke exposure, and desire to lose weight) and suboptimal, borderline, and poor glycemic control using nationally representative secondary data (NHANES III and 1999-2004). We also investigated the consequences of suboptimal, borderline, and poor glycemic control in terms of HRQOL, employment status, absenteeism, and mortality.

Our findings suggest that the number of hours of work and possibly the type of occupation an adult with diabetes has does affect their level of glycemic control. However, whether the adult with diabetes is exposed to secondhand smoke or whether he or she wishes to lose weight may not have a significant bearing on their degree of glycemic control and further studies are needed to define these relationships accurately. In addition, we found that poor mental health to be among those with poor glycemic control yet better for those with suboptimal glycemic control. Furthermore, individuals with poor glycemic control may be more likely to have greater days of inactivity. Interestingly, although greater number of days of poor mental health and inactivity were reported among those with poor glycemic control, there was no statistically significant relationship between employment status and missed work days and glycemic control. Thus, our data suggest that individuals with poor glycemic control are attending work yet probably are not performing at their optimal level; in other words, there may be greater

preseenteism among adults with poor glycemic control. Finally, our findings imply that 
their may be a linear relationship between $\mathrm{HbAlc}$ levels and mortality risk among males.

In addition, strict glycemic control appears to be protective of mortality after a 6 to 12

year follow-up among adults with diabetes, even after controlling for potential

confounders including comorbidity, which contradicts recent reports. ${ }^{242}$ These main

findings from this study summarized in figure 2 (shown below).

Figure 2. A diagrammatic view of main study findings

Research Question 1:

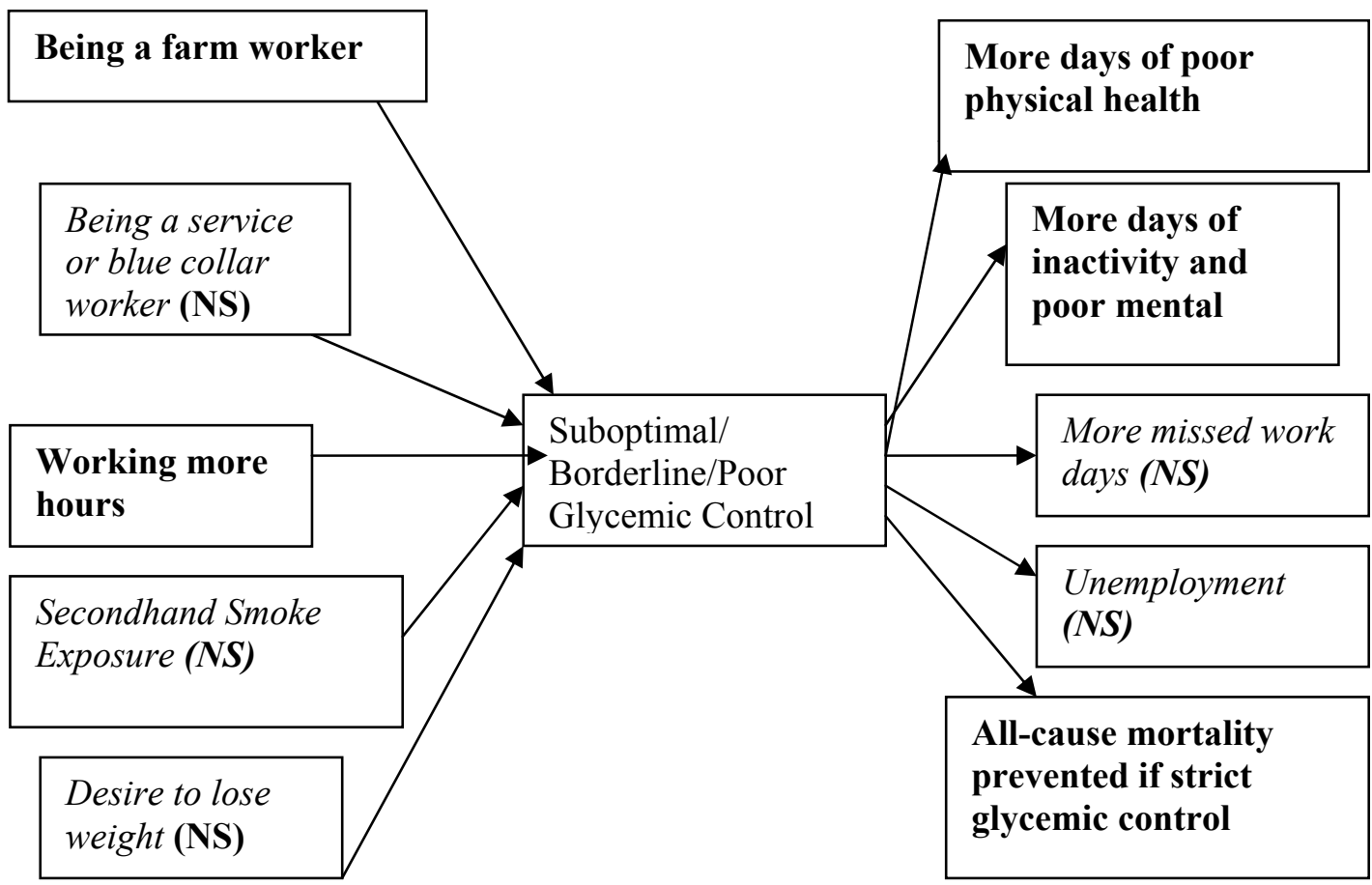

Note: NS=non-significant; statistically significant findings appear in bold. 


\section{Figure 3. A diagrammatic view of key recommendations for good glycemic control}

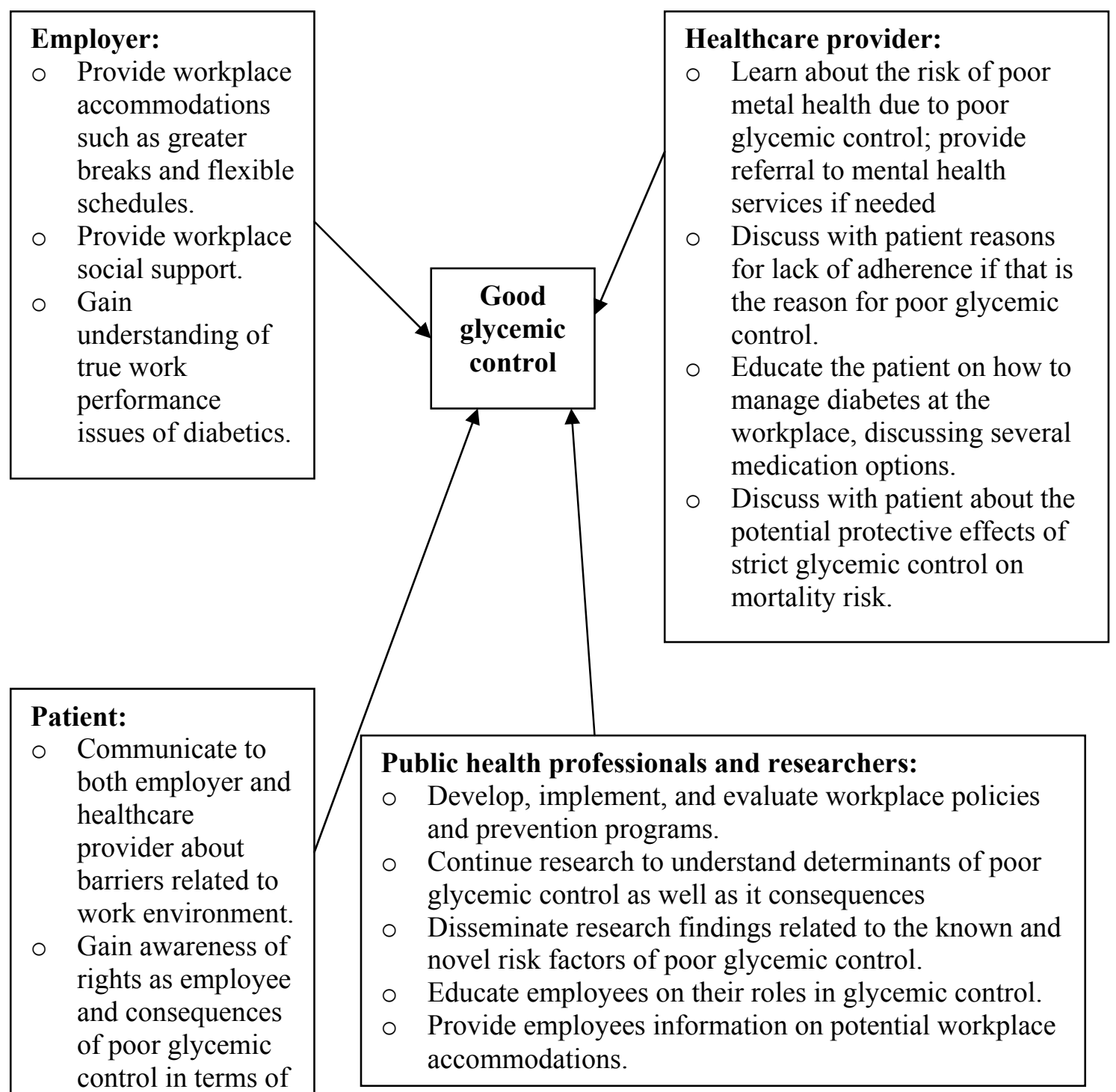


There are several implications and recommendations for employers, adults with diabetes and their primary healthcare providers, and public health advocates, professionals and researchers from our study findings. The key recommendations are presented in figure 3.

\section{Employers}

Given that work related factors appear to affect glycemic control, it is important to raise awareness among employers regarding issues that their employees with diabetes may have in terms of managing their diabetes. Employers should understand the benefits of having certain minor yet important workplace accommodations for their employees with diabetes. Such workplace accommodations, and as recommended by the American Diabetes Association, ${ }^{266}$ may include healthier food choices at work, private places to check blood sugars or self-administer insulin, greater or more frequent breaks, more flexible work-schedules including the option to take time off to be able to visit their diabetes educator or health care provider, and even provision of diabetes educational materials and/or educational items or prevention programs on good overall health practices such as exercise and diet. ${ }^{267}$

Provision of workplace accommodations may be contingent on the workers with diabetes telling their employer of their illness. However, because of fear of work discrimination, the individual with diabetes may choose not to disclose their illness to the employer, even though such disclosure could result in employer support and workplace accommodations. ${ }^{268,269}$ In fact, compared to individuals with other chronic conditions, individuals with diabetes may be particularly less likely to disclose their medical status 
for various reasons such as loss of job, rejection by co-workers and employer, and discrimination. ${ }^{268}$ The employer may discriminate against an employee with diabetes due to the false perception that the employee cannot carry on a normal workload, may have reduced performance due to ill health, may have episodes of hypoglycemia or hyperglycemia that may lead to hazardous workplace situations, or the belief that these employees with diabetes may require costly special workplace accommodations. However, these reasons for discrimination are perhaps without merit. For example, studies have shown that individuals with diabetes perform well or even better in the job compared to individuals without diabetes. ${ }^{224,270}$ In addition, hypoglycemic events during work are unlikely ${ }^{262,271}$ and if they do occur, they typically do not result in any significant disruptions at work, ${ }^{272}$ and are particularly less common among adults with type 2 diabetes. ${ }^{208,273}$ Thus, the notion that diabetes is an impediment in the workplace has to be rethought. However, as suggested from our findings, it may be that poor glycemic control, not necessarily diagnosis of diabetes, may results in poor work performance and productivity, given the reported greater days of inactivity. This further highlight's the importance of workplace policies and programs aimed at supporting effective diabetes management.

Furthermore, the importance of social support in the workplace needs to be underscored, since social support, especially among Hispanics, has resulted in improvements in diabetes management behavior. ${ }^{177,}{ }^{179}$ In addition, social support provided by promotoras (i.e. community health workers) for farm workers has also been associated with reduced $\mathrm{HbAlc}$ levels as well as greater participation in diabetes support groups. $^{171}$ 
According to the American Diabetes Association, good diabetes control costs approximately \$24 a month, a much lower expense than the \$115 a month it would cost the employer in medical expenditures and loss in productivity to have diabetic workers in poor health, including those experiencing diabetic complications. ${ }^{274}$ Thus, it may be costeffective for employers to understand the important role they in the diabetes management of their employees diagnosed with diabetes. From an economic point of view that stipulates that individual earnings are a result of the individual's level of productivity, ${ }^{275}$ inadequate diabetes management may result in lower income, which could further hinder the ability of the diabetic patient to purchase pharmacological agents or meals essential for proper diabetes management.

Individuals with diabetes and their healthcare providers

Given that long work hours or inflexible schedules are sometimes inevitable, certain accommodations should be considered for individuals with type 2 diabetes to make sure their blood glucose levels are normalized as much as possible. These accommodations may involve taking medications that have various duration of action or extended release properties based on work schedule. This may require keeping logs of the different work schedules and when each meal would be eaten, especially for workers with changing schedules. ${ }^{276,277}$ For insulin-taking individuals with inflexible work schedules, awareness of the implications of such work schedule is important so that when speaking with their health care provider, the employee may be able to ask the physician about this concern and what actions should be taken to mitigate such consequences; this may include switching to different diabetes medication options and dosing regimens. ${ }^{278}$ 
Moreover, because some patients may be fearful of hypoglycemic episodes, which may lead to lack of adherence to treatment regimens, ${ }^{208,272}$ patient education and strategies to prevent hypoglycemia is imperative. For example, the integration of coping strategies in order to provide the diabetic patient with a "sense of control" may be of great benefit in diabetes management programs. ${ }^{208}$ Furthermore, it may be beneficial to have diabetes self-management programs, particularly among those that are fearful of hypoglycemia as a consequence of strict diabetes regimens, as these programs have been shown to reduce the risk of hypoglycemia. ${ }^{279}$

In addition, health care provider or diabetes educators should take into account barriers to healthy food choices for an individual with diabetes, particularly barriers due to work factors such as break times, work hours, and food availability and/or place to store/prepare foods. ${ }^{280}$ Given that eating in a scheduled pattern or timely manner may not be feasible in certain occupations no matter how planned it is, it may be imperative to provide more tailored diabetes education. For example, one approach may be to educate how to adjust insulin based on the carbohydrate intake rather than carbohydrate intake based on insulin administration. A study among the Australian population showed that tailored eating program can result in benefits such as reduced HbAlc levels, minimal hypoglycemic episodes, and improved quality of life. ${ }^{281}$

Diabetes management has been associated with a degree of burden and consequently perceived worse quality of life ${ }^{282}$ and techniques such as cognitive behavioral therapy, coping skills training, counseling, and patient empowerment programs have been found to be successful among individuals with diabetes. ${ }^{283-285}$ Thus, the use of these techniques in diabetes management programs is recommended. These 
strategies may help off-set the feelings of emotional distress among adults with diabetes and consequent risk of poor adherence and poor glycemic control. ${ }^{286}$ However, since it has been suggested that primary health care providers may not know how to properly counsel a diabetic patient with poor mental health, ${ }^{286}$ referrals to professionals in the psychology or mental health field is recommended.

\section{$\underline{\text { Public health advocates and professionals }}$}

Given the association between glycemic control and work-related factors and the fact that much of a person's time is spent at work, the workplace should be an avenue in which to educate both employers and employees with diabetes about diabetes management. Workplace health programs have in fact been shown to be effective at improving health among employees. ${ }^{287}$ Furthermore, increasing the prevalence of health promotion programs in the US is one of the goals of Healthy People $2010 .{ }^{288}$ However, it is important that these worksite health promotion programs be available to all workers, which appears to not be the current situation. For example, one study found that nonprofessional, Black, and less educated workers were less likely to report having received any sort of health promotion in the worksite. ${ }^{289}$ In addition, when these worksite health programs are available, certain workers, such as those workers receiving a higher salary and white collar workers appear more likely to participate than other workers. ${ }^{290,291}$ The lack of participation in these worksite programs may be in part due to the work schedule of these individuals. ${ }^{292}$ Public health professionals should also work to address discrimination encountered by adults with diabetes that can hinder the proper management of diabetes. 
To offset such discrimination, awareness of the rights of workers with diabetes and education of both the employers and employees should be required. This should entail proper education on the reality of living with diabetes as well as the legal terminology when speaking about individuals with chronic conditions including what the term "disability" means. ${ }^{293}$ When individuals with diabetes argue that they need special accommodations, assuming these are considered "reasonable", the employers should provide them in accordance with the Americans with Disabilities Act (ADA). ${ }^{267}$ However, since this act is based on individuals with more clear cut disabilities such as blindness, diabetes is often times not considered a hindering disability or covered by this act, and there is lack of clarity of the rights of adults with diabetes. ${ }^{267,293}$ Thus, changes to the ADA, such as clear statements regarding what constitutes a reasonable and justifiable accommodation for an adult with diabetes should be implemented. ${ }^{267}$ Furthermore, policies such as insurance reimbursement of nutrition education services or free nutrition education among adults without insurance may prove to be beneficial since research has shown that adults with diabetes may not know the importance of nutrition and have been shown to not be compliant with visiting their dietitian; yet proper nutrition is key to diabetes management and improved patient prognosis. ${ }^{159}$

\section{$\underline{\text { Future research }}$}

Further research is needed however to clearly understand the mechanisms involved in the relationships between work hours, the type of occupation, and glycemic control. Such studies would benefit from including surveys or questionnaires that ask about several work related characteristics, such as shift-work, night shift, number of 
breaks, degree of flexibility to take time off from work, and usual tasks at work. In addition, studies on the relationship between presenteeism and glycemic control are needed. However this is contingent on the availability and feasibility of reliable measures of presenteeism.

Moreover, research is needed to understand the mechanisms involved in the relationship between poor mental health and glycemic control. Finally, prospective and longitudinal studies assessing the risk of mortality due to various degrees of glycemic control among a nationally representative sample of adults are needed. These studies would benefit from recording data on the number of hospitalizations, diabetes complications, changes in diabetes treatment, and episodes of hypo and hyperglycemia, as well as repeated measures of $\mathrm{HbAlc}$ tests. 


\section{LIST OF REFERENCES}

1. Centers for Disease Control and Prevention. National diabetes fact sheet: general information and national estimates on diabetes in the United States, 2005. Atlanta, GA: U.S. Department of Health and Human Services, Centers for Disease Control and Prevention, 2005.

2. Boyle JP, Honeycutt AA, Narayan KM, Hoerger TJ, Geiss LS, Chen H, et al. Projection of diabetes burden through 2050: impact of changing demography and disease prevalence in the U.S. Diabetes Care 2001;24(11):1936-40.

3. Wild S, Roglic G, Green A, Sicree R, King H. Global prevalence of diabetes: estimates for the year 2000 and projections for 2030. Diabetes Care 2004;27(5):1047-53.

4. Honeycutt AA, Boyle JP, Broglio KR, Thompson TJ, Hoerger TJ, Geiss LS, et al. A dynamic Markov model for forecasting diabetes prevalence in the United States through 2050. Health Care Manag Sci 2003;6(3):155-64.

5. Jemal A, Ward E, Hao Y, Thun M. Trends in the leading causes of death in the United States, 1970-2002. Jama 2005;294(10):1255-9.

6. Centers for Disease Control and Prevention. National Center for Health Statistics. Faststats. Leading cause of death. May 2009. Available from:

http://www.cdc.gov/nchs/FASTATS/lcod.htm. (accessed June 20, 2009)

7. Centers for Disease Control and Prevention. Diabetes. Successs and opportunities for population-based prevention and control: at a glance 2009. Available from: http://diabetes.niddk.nih.gov/dm/pubs/diagnosis/ (accessed November 10, 2008)

8. National Institute of Diabetes and Digestive and Kidney Diseases. National Insitute of Health. National Diabetes Information Clearinghouse (NDIC). Diagnosis of Diabetes. October 2008. Available from:

http://diabetes.niddk.nih.gov/dm/pubs/diagnosis/ (accessed November 10, 2008

9. Report of the expert committee on the diagnosis and classification of diabetes mellitus. Diabetes Care 2003;26 Suppl 1:S5-20.

10. LeRoith D, Smith DO. Monitoring glycemic control: the cornerstone of diabetes care. Clin Ther 2005;27(10):1489-99.

11. Delamater A. Clinical use of hemoglobin A1c to improve diabetes management. Clinical Diabetes 2006;24(1):6-8. 
12. Hom FG, Ettinger B, Lin MJ. Comparison of serum fructosamine vs glycohemoglobin as measures of glycemic control in a large diabetic population. Acta Diabetol 1998;35(1):48-51.

13. The American Association of Clinical Endocrinologists Medical Guidelines for the Management of Diabetes Mellitus: the AACE system of intensive diabetes selfmanagement--2000 update. Endocr Pract 2000;6(1):43-84.

14. Valentine WJ, Palmer AJ, Nicklasson L, Cobden D, Roze S. Improving life expectancy and decreasing the incidence of complications associated with type 2 diabetes: a modelling study of HbA1c targets. Int J Clin Pract 2006;60(9):1138-45.

15. Maney M, Tseng CL, Safford MM, Miller DR, Pogach LM. Impact of selfreported patient characteristics upon assessment of glycemic control in the Veterans Health Administration. Diabetes Care 2007;30(2):245-51.

16. Chaudhry SI, Berlowitz DR, Concato J. Do age and comorbidity affect intensity of pharmacological therapy for poorly controlled diabetes mellitus? J Am Geriatr Soc 2005;53(7):1214-6.

17. Pogach LM, Brietzke SA, Cowan CL, Jr., Conlin P, Walder DJ, Sawin CT. Development of evidence-based clinical practice guidelines for diabetes: the Department of Veterans Affairs/Department of Defense guidelines initiative. Diabetes Care 2004;27 Suppl 2:B82-9.

18. Standards of medical care in diabetes--2007. Diabetes Care 2007;30 Suppl 1:S4S41.

19. Klein R. Hyperglycemia and microvascular and macrovascular disease in diabetes. Diabetes Care 1995;18(2):258-68.

20. Turner RC, Millns H, Neil HA, Stratton IM, Manley SE, Matthews DR, et al. Risk factors for coronary artery disease in non-insulin dependent diabetes mellitus: United Kingdom Prospective Diabetes Study (UKPDS: 23). Bmj 1998;316(7134):823-8.

21. Tsai C, Hayes C, Taylor GW. Glycemic control of type 2 diabetes and severe periodontal disease in the US adult population. Community Dent Oral Epidemiol 2002;30(3):182-92.

22. Klein R, Klein BE, Moss SE. Relation of glycemic control to diabetic microvascular complications in diabetes mellitus. Ann Intern Med 1996;124(1 Pt 2):90-6.

23. King DE, Mainous AG, 3rd, Buchanan TA, Pearson WS. C-reactive protein and glycemic control in adults with diabetes. Diabetes Care 2003;26(5):1535-9. 
24. Clark CM, Jr., Fradkin JE, Hiss RG, Lorenz RA, Vinicor F, Warren-Boulton E. The National Diabetes Education Program, changing the way diabetes is treated: comprehensive diabetes care. Diabetes Care 2001;24(4):617-8.

25. Saydah SH, Fradkin J, Cowie CC. Poor control of risk factors for vascular disease among adults with previously diagnosed diabetes. Jama 2004;291(3):335-42.

26. Saaddine JB, Engelgau MM, Beckles GL, Gregg EW, Thompson TJ, Narayan KM. A diabetes report card for the United States: quality of care in the 1990s. Ann Intern Med 2002;136(8):565-74.

27. Hoerger TJ, Segel JE, Gregg EW, Saaddine JB. Is glycemic control improving in U.S. adults? Diabetes Care 2008;31(1):81-6.

28. NCHS. Analytic and Reporting Guidelines. The National Health and Nutrition Examination Survey (NHANES) September, 2006. National Center for Health Statistics Centers for Disease Control and Prevention Hyattsville, Maryland. Available from: http://www.cdc.gov/nchs/data/nhanes/nhanes 03 04/nhanes analytic guidelines dec 20 05.pdf (accessed January 15 2007).

29. Hennessy CH, Moriarty DG, Zack MM, Scherr PA, Brackbill R. Measuring health-related quality of life for public health surveillance. Public Health Rep 1994;109(5):665-72.

30. Harris MI, Eastman RC, Cowie CC, Flegal KM, Eberhardt MS. Racial and ethnic differences in glycemic control of adults with type 2 diabetes. Diabetes Care 1999;22(3):403-8.

31. Fan T, Koro CE, Fedder DO, Bowlin SJ. Ethnic disparities and trends in glycemic control among adults with type 2 diabetes in the U.S. from 1988 to 2002. Diabetes Care 2006;29(8):1924-5.

32. Saydah S, Cowie C, Eberhardt MS, De Rekeneire N, Narayan KM. Race and ethnic differences in glycemic control among adults with diagnosed diabetes in the United States. Ethn Dis 2007;17(3):529-35.

33. Shorr RI, Franse LV, Resnick HE, Di Bari M, Johnson KC, Pahor M. Glycemic control of older adults with type 2 diabetes: findings from the Third National Health and Nutrition Examination Survey, 1988-1994. J Am Geriatr Soc 2000;48(3):264-7.

34. Mackenzie T, Brooks B, O'Connor G. Beverage intake, diabetes, and glucose control of adults in America. Ann Epidemiol 2006;16(9):688-91. 
35. Suh DC, Kim CM, Choi IS, Plauschinat CA. Comorbid conditions and glycemic control in elderly patients with type 2 diabetes mellitus, 1988 to 1994 to 1999 to 2004 . J Am Geriatr Soc 2008;56(3):484-92.

36. Ong KL, Cheung BM, Wong LY, Wat NM, Tan KC, Lam KS. Prevalence, treatment, and control of diagnosed diabetes in the U.S. National Health and Nutrition Examination Survey 1999-2004. Ann Epidemiol 2008;18(3):222-9.

37. Heisler M, Faul JD, Hayward RA, Langa KM, Blaum C, Weir D. Mechanisms for racial and ethnic disparities in glycemic control in middle-aged and older Americans in the health and retirement study. Arch Intern Med 2007;167(17):1853-60.

38. Van Tilburg MA, McCaskill CC, Lane JD, Edwards CL, Bethel A, Feinglos MN, et al. Depressed mood is a factor in glycemic control in type 1 diabetes. Psychosom Med 2001;63(4):551-5.

39. Weinger K, Jacobson AM. Psychosocial and quality of life correlates of glycemic control during intensive treatment of type 1 diabetes. Patient Educ Couns 2001;42(2):123-31.

40. Hertz RP, McDonald M, Unger AN, Lustik MB. Racial and ethnic disparities in the prevalence and management of cardiovascular risk factors in the United States workforce. J Occup Environ Med 2007;49(10):1165-75.

41. Kroenke CH, Spiegelman D, Manson J, Schernhammer ES, Colditz GA, Kawachi I. Work characteristics and incidence of type 2 diabetes in women. Am J Epidemiol 2007;165(2):175-83.

42. Kawakami N, Akachi K, Shimizu H, Haratani T, Kobayashi F, Ishizaki M, et al. Job strain, social support in the workplace, and haemoglobin A1c in Japanese men. Occup Environ Med 2000;57(12):805-9.

43. Houston TK, Person SD, Pletcher MJ, Liu K, Iribarren C, Kiefe CI. Active and passive smoking and development of glucose intolerance among young adults in a prospective cohort: CARDIA study. Bmj 2006;332(7549):1064-9.

44. Trief PM, Aquilino C, Paradies K, Weinstock RS. Impact of the work environment on glycemic control and adaptation to diabetes. Diabetes Care 1999;22(4):569-74.

45. de Alba Garcia JG, Rocha AL, Lopez I, Baer RD, Dressler W, Weller SC. "Diabetes is my companion": lifestyle and self-management among good and poor control Mexican diabetic patients. Soc Sci Med 2007;64(11):2223-35. 
46. Netterstrom B, Kristensen TS, Damsgaard MT, Olsen O, Sjol A. Job strain and cardiovascular risk factors: a cross sectional study of employed Danish men and women. Br J Ind Med 1991;48(10):684-9.

47. Kawakami N, Araki S, Hayashi T, Masumoto T. Relationship between perceived job-stress and glycosylated hemoglobin in white-collar workers. Ind Health 1989;27(4):149-54.

48. Belkic KL, Landsbergis PA, Schnall PL, Baker D. Is job strain a major source of cardiovascular disease risk? Scand J Work Environ Health 2004;30(2):85-128.

49. Marmot MG, Bosma H, Hemingway H, Brunner E, Stansfeld S. Contribution of job control and other risk factors to social variations in coronary heart disease incidence. Lancet 1997;350(9073):235-9.

50. Schnall PL, Landsbergis PA, Pickering TG, Schwartz JE. Perceived job stress, job strain, and hypertension. Am J Public Health 1994;84(2):320-1.

51. Schnall PL, Landsbergis PA, Baker D. Job strain and cardiovascular disease. Annu Rev Public Health 1994;15:381-411.

52. Soule J, Egede L. Challenges of Managing Diabetes in Commercial Truck Drivers. Clinical Diabetes 2007;25(3):110-111.

53. Cobb S, Rose RM. Hypertension, peptic ulcer, and diabetes in air traffic controllers. Jama 1973;224(4):489-92.

54. Morikawa Y, Nakagawa H, Ishizaki M, Tabata M, Nishijo M, Miura K, et al. Ten-year follow-up study on the relation between the development of non-insulindependent diabetes mellitus and occupation. Am J Ind Med 1997;31(1):80-4.

55. Kawakami N, Araki S, Takatsuka N, Shimizu H, Ishibashi H. Overtime, psychosocial working conditions, and occurrence of non-insulin dependent diabetes mellitus in Japanese men. J Epidemiol Community Health 1999;53(6):359-63.

56. Nakanishi N, Nishina K, Yoshida H, Matsuo Y, Nagano K, Nakamura K, et al. Hours of work and the risk of developing impaired fasting glucose or type 2 diabetes mellitus in Japanese male office workers. Occup Environ Med 2001;58(9):569-74.

57. Baker J, Scragg R, Metcalf P, Dryson E. Diabetes mellitus and employment: survey of a New Zealand workforce. Diabet Med 1993;10(4):359-61.

58. Pitsavos C, Panagiotakos DB, Chrysohoou C, Skoumas J, Tzioumis K, Stefanadis $\mathrm{C}$, et al. Association between exposure to environmental tobacco smoke and the development of acute coronary syndromes: the CARDIO2000 case-control study. Tob Control 2002;11(3):220-5. 
59. Eliasson B. Cigarette smoking and diabetes. Prog Cardiovasc Dis 2003;45(5):40513.

60. Madsbad S, McNair P, Christensen MS, Christiansen C, Faber OK, Binder C, et al. Influence of smoking on insulin requirement and metbolic status in diabetes mellitus. Diabetes Care 1980;3(1):41-3.

61. Chase HP, Garg SK, Marshall G, Berg CL, Harris S, Jackson WE, et al. Cigarette smoking increases the risk of albuminuria among subjects with type I diabetes. Jama 1991;265(5):614-7.

62. Morrish NJ, Stevens LK, Fuller JH, Jarrett RJ, Keen H. Risk factors for macrovascular disease in diabetes mellitus: the London follow-up to the WHO Multinational Study of Vascular Disease in Diabetics. Diabetologia 1991;34(8):590-4.

63. Lundman BM, Asplund K, Norberg A. Smoking and metabolic control in patients with insulin-dependent diabetes mellitus. J Intern Med 1990;227(2):101-6.

64. Chaturvedi N, Stephenson JM, Fuller JH. The relationship between smoking and microvascular complications in the EURODIAB IDDM Complications Study. Diabetes Care 1995;18(6):785-92.

65. Hayashino Y, Fukuhara S, Okamura T, Yamato H, Tanaka H, Tanaka T, et al. A prospective study of passive smoking and risk of diabetes in a cohort of workers: the High-Risk and Population Strategy for Occupational Health Promotion (HIPOP-OHP) study. Diabetes Care 2008;31(4):732-4.

66. Makimattila S, Nikkila K, Yki-Jarvinen H. Causes of weight gain during insulin therapy with and without metformin in patients with Type II diabetes mellitus. Diabetologia 1999;42(4):406-12.

67. Fritsche A, Haring H. At last, a weight neutral insulin? Int J Obes Relat Metab Disord 2004;28 Suppl 2:S41-6.

68. Nathan DM. Clinical practice. Initial management of glycemia in type 2 diabetes mellitus. N Engl J Med 2002;347(17):1342-9.

69. Daly A. Use of Insulin and Weight Gain: Optimizing Diabetes Nutrition Therapy. Journal of the American Dietetic Association 2007;107:1386-1393.

70. Friedman S, Vila G, Timsit J, Mouren-Simeoni M. Eating disorders and insulindependent diabetes mellitus (IDDM): relationships with glycaemic control and somatic complications. Acta Psychiatrica Scandinavica 1998;97:206-212. 
71. Wing RR, Nowalk MP, Marcus MD, Koeske R, Finegold D. Subclinical eating disorders and glycemic control in adolescents with type I diabetes. Diabetes Care 1986;9(2):162-7.

72. Daneman D, Rodin G, Jones P, Colton A, Rydall S, Maharai S, et al. Eating Disorders in Adolescent Girls and Young Adults Women with Type 1 Diabetes. Diabetes Sprectum 2002;15(2):83-105.

73. Lawrence JM, Liese AD, Liu L, Dabelea D, Anderson A, Imperatore G, et al. Weight-loss practices and weight-related issues among youth with type 1 or type 2 diabetes. Diabetes Care 2008;31(12):2251-7.

74. CDC. Healthy People 2010. Available from: http://www.healthypeople.gov/About/goals.htm (accessed April 20, 2006).

75. Centers for Disease Control and Prevention. Health Related Quality of Life. 2007. Available from: http://www.cdc.gov/hrqol/ (accessed April 20, 2006).

76. Jiang Y, Hesser JE. Associations between health-related quality of life and demographics and health risks. Results from Rhode Island's 2002 behavioral risk factor survey. Health Qual Life Outcomes 2006;4:14.

77. Jacobson AM. Impact of improved glycemic control on quality of life in patients with diabetes. Endocr Pract 2004;10(6):502-8.

78. Centers for Disease Control and Prevention. Measuring Healthy Days. Atlanta, Georgia: CDC, November 2000. Available from:

http://www.cdc.gov/hrqol/pdfs/mhd.pdf (accessed May 2, 2008)

79. Rubin RR, Peyrot M. Quality of life and diabetes. Diabetes Metab Res Rev 1999;15(3):205-18.

80. de Sonnaville JJ, Snoek FJ, Colly LP, Deville W, Wijkel D, Heine RJ. Well-being and symptoms in relation to insulin therapy in type 2 diabetes. Diabetes Care 1998;21(6):919-24.

81. Lloyd A, Sawyer W, Hopkinson P. Impact of long-term complications on quality of life in patients with type 2 diabetes not using insulin. Value Health 2001;4(5):392-400.

82. Wexler DJ, Grant RW, Wittenberg E, Bosch JL, Cagliero E, Delahanty L, et al. Correlates of health-related quality of life in type 2 diabetes. Diabetologia 2006;49(7):1489-97.

83. Lau CY, Qureshi AK, Scott SG. Association between glycaemic control and quality of life in diabetes mellitus. J Postgrad Med 2004;50(3):189-93; discussion 194. 
84. Hoey H, Aanstoot HJ, Chiarelli F, Daneman D, Danne T, Dorchy H, et al. Good metabolic control is associated with better quality of life in 2,101 adolescents with type 1 diabetes. Diabetes Care 2001;24(11):1923-8.

85. Lange LJ, Piette JD. Perceived health status and perceived diabetes control: psychological indicators and accuracy. J Psychosom Res 2005;58(2):129-37.

86. Influence of intensive diabetes treatment on quality-of-life outcomes in the diabetes control and complications trial. Diabetes Care 1996;19(3):195-203.

87. White NH, Cleary PA, Dahms W, Goldstein D, Malone J, Tamborlane WV. Beneficial effects of intensive therapy of diabetes during adolescence: outcomes after the conclusion of the Diabetes Control and Complications Trial (DCCT). J Pediatr 2001;139(6):804-12.

88. Aalto AM, Uutela A, Aro AR. Health related quality of life among insulindependent diabetics: disease-related and psychosocial correlates. Patient Educ Couns 1997;30(3):215-25.

89. Larsson D, Lager I, Nilsson PM. Socio-economic characteristics and quality of life in diabetes mellitus--relation to metabolic control. Scand J Public Health 1999;27(2):101-5.

90. Mitchell BD, Stern MP, Haffner SM, Hazuda HP, Patterson JK. Functional impairment in Mexican Americans and non-Hispanic whites with diabetes. J Clin Epidemiol 1990;43(4):319-27.

91. Glasgow RE, Ruggiero L, Eakin EG, Dryfoos J, Chobanian L. Quality of life and associated characteristics in a large national sample of adults with diabetes. Diabetes Care 1997;20(4):562-7.

92. Toossi M. Labor force projections to 2012: the graying of the U.S. workforce. Monthly Labor Review: Feburary, 2004.37-57.

93. National Institute of Health. National Diabetes Information Clearing House. National Diabetes Statistics, 2007. Available from: http://diabetes.niddk.nih.gov/DM/PUBS/statistics/ (accessed January 20, 2009)

94. Kessler RC, Greenberg PE, Mickelson KD, Meneades LM, Wang PS. The effects of chronic medical conditions on work loss and work cutback. J Occup Environ Med 2001;43(3):218-25.

95. Boyd A. Employment traps: Corruption in the workplace. Management review 1997;86:9. 
96. Danna K, Griffin R. Health and well-being in the workplace: A review and synthesis of the literature. Journal of Management 1999;25:357-384.

97. Robinson N, Yateman NA, Protopapa LE, Bush L. Employment problems and diabetes. Diabet Med 1990;7(1):16-22.

98. Vijan S, Hayward RA, Langa KM. The impact of diabetes on workforce participation: results from a national household sample. Health Serv Res 2004;39(6 Pt 1):1653-69.

99. Baker J, Scragg R, Metcalf P, Dryson E. Diabetes mellitus and employment: is there discrimination in the workplace? Diabet Med 1993;10(4):362-5.

100. Ng YC, Jacobs P, Johnson JA. Productivity losses associated with diabetes in the US. Diabetes Care 2001;24(2):257-61.

101. Ramsey S, Summers KH, Leong SA, Birnbaum HG, Kemner JE, Greenberg P. Productivity and medical costs of diabetes in a large employer population. Diabetes Care 2002;25(1):23-9.

102. Testa MA, Simonson DC. Health economic benefits and quality of life during improved glycemic control in patients with type 2 diabetes mellitus: a randomized, controlled, double-blind trial. Jama 1998;280(17):1490-6.

103. Tunceli K, Bradley CJ, Lafata JE, Pladevall M, Divine GW, Goodman AC, et al. Glycemic control and absenteeism among individuals with diabetes. Diabetes Care 2007;30(5):1283-5.

104. Licciardone JC, Kotsanos JG, Brinkman-Kaplan V, Cooper T, Jordan JE, Wishner KL. Resource utilization and work or school loss reported by patients with diabetes: experience in diabetes training programs. Am J Manag Care 1997;3(5):777-82.

105. Kovesdy CP, Sharma K, Kalantar-Zadeh K. Glycemic control in diabetic CKD patients: where do we stand? Am J Kidney Dis 2008;52(4):766-77.

106. Menon V, Greene T, Pereira AA, Wang X, Beck GJ, Kusek JW, et al. Glycosylated hemoglobin and mortality in patients with nondiabetic chronic kidney disease. J Am Soc Nephrol 2005;16(11):3411-7.

107. Kalantar-Zadeh K, Kopple JD, Regidor DL, Jing J, Shinaberger CS, Aronovitz J, et al. A1C and survival in maintenance hemodialysis patients. Diabetes Care 2007;30(5):1049-55. 
108. Khaw KT, Wareham N, Bingham S, Luben R, Welch A, Day N. Association of hemoglobin A1c with cardiovascular disease and mortality in adults: the European prospective investigation into cancer in Norfolk. Ann Intern Med 2004;141(6):413-20.

109. Scheidt-Nave C, Barrett-Connor E, Wingard DL, Cohn BA, Edelstein SL. Sex differences in fasting glycemia as a risk factor for ischemic heart disease death. Am J Epidemiol 1991;133(6):565-76.

110. Park S, Barrett-Connor E, Wingard DL, Shan J, Edelstein S. GHb is a better predictor of cardiovascular disease than fasting or postchallenge plasma glucose in women without diabetes. The Rancho Bernardo Study. Diabetes Care 1996;19(5):450-6.

111. Schwedler SB, Metzger T, Schinzel R, Wanner C. Advanced glycation end products and mortality in hemodialysis patients. Kidney Int 2002;62(1):301-10.

112. Eshaghian S, Horwich TB, Fonarow GC. An unexpected inverse relationship between $\mathrm{HbAlc}$ levels and mortality in patients with diabetes and advanced systolic heart failure. Am Heart J 2006;151(1):91.

113. NCHS. NHANES III Linked Mortality 2005. Available from : http://www.cdc.gov/nchs/r\&d/nchs datalinkage/nhanes data_linkage activities.htm (accessed September 15, 2005).

114. National Center for Health Statistics. National Health and Nutrition Examination Survey. Documentation, Codebook, and Frequencies. MEC Laboratory Component. Glycohemoglobin. Survey Years 2003-2004. January 2006. Available from: http://www.cdc.gov/nchs/data/nhanes/nhanes 03 04/110 c.pdf (accessed April 10, 2008)

115. Bish CL, Michels Blanck H, Maynard LM, Serdula MK, Thompson NJ, Kettel Khan L. Health-related quality of life and weight loss among overweight and obese U.S. adults, 2001 to 2002. Obesity (Silver Spring) 2006;14(11):2042-53.

116. Zahran HS, Kobau R, Moriarty DG, Zack MM, Holt J, Donehoo R. Health-related quality of life surveillance--United States, 1993-2002. MMWR Surveill Summ 2005;54(4):1-35.

117. National Center for Health Statistics, Office of Analysis and Epidemiology. The Third National Health and Nutrition Examination Survey (NHANES III) Linked Mortality File, mortality follow-up through 2006: matching methodology. 2009. Available from: http://www.cdc.gov/nchs/data/datalinkage/matching methodology nhanes3 final.pdf (accessed February 2, 2009)

118. Carr D. Overtime work: an expanded view. Monthly Labor Review 1986;109(11). 
119. Nardone T. Part-time workers: who are they? Monthly Labor Review $1986 ; 109(2)$.

120. NCHS. Classified Index of Industries and Occupations, 1989. Washington, DC: US Department of Health and Human Services; 1989.

121. Krieger N, Barbeau EM, Soobader MJ. Class matters: U.S. versus U.K. measures of occupational disparities in access to health services and health status in the 2000 U.S. National Health Interview Survey. Int J Health Serv 2005;35(2):213-36.

122. Biochemical verification of tobacco use and cessation. Nicotine Tob Res 2002;4(2):149-59.

123. Fleming LE, Levis S, LeBlanc WG, Dietz NA, Arheart KL, Wilkinson JD, et al. Earlier age at menopause, work, and tobacco smoke exposure. Menopause 2008;15(6):1103-8.

124. Arheart KL, Lee DJ, Fleming LE, LeBlanc WG, Dietz NA, McCollister KE, et al. Accuracy of self-reported smoking and secondhand smoke exposure in the US workforce: the National Health and Nutrition Examination Surveys. J Occup Environ Med 2008;50(12):1414-20.

125. NCHS. Plan and operation of the Third National Health and Nutrition Examination Survey, 1988-94. Series 1: programs and collection procedures. Vital Health Stat 1 1994(32):1-407.

126. Hosmer DW, S L. Applied Logist Regression . Second Edition John Wiley \& Sons, Inc New York, NY. 2000.

127. Kleinbaum DG, Kupper, L. L., Muller, K. E., Nizam, A. Applied Regression Analysis and Other Multivariable Methods,. (3rd. Ed.). ed. Pacific Grove, CA: Duxbury Press.; 1998.

128. PV L. Module 8: Selecting regression models. Master of Applied Statistics. Regressino and analysis of variance 2007. Available from: http://statmaster/sdu/dk/courses/st111 (accessed February 12, 2008)

129. Salinas JJ, Peek MK. Work experience and gender differences in chronic disease risk in older Mexicans. Ann Epidemiol 2008;18(8):628-30.

130. Sex differences in the mortality associated with insulin-dependent diabetes mellitus in four countries. The Diabetes Epidemiology Research International (DERI) Study. Am J Epidemiol 1991;133(6):577-84. 
131. Kleinbaum D, Kupper L, Morgenstern H. Epidemiologic Research Principles and Quantitative Methods. . John Wiley and Sons; 1982.

132. Baron RM, Kenny DA. The moderator-mediator variable distinction in social psychological research: conceptual, strategic, and statistical considerations. J Pers Soc Psychol 1986;51(6):1173-82.

133. Gardner W, Mulvey EP, Shaw EC. Regression analyses of counts and rates: Poisson, overdispersed Poisson, and negative binomial models. Psychol Bull 1995;118(3):392-404.

134. Ver Hoef JM, Boveng PL. Quasi-Poisson vs. negative binomial regression: how should we model overdispersed count data? Ecology 2007;88(11):2766-72.

135. Nagelkerk J, Reick K, Meengs L. Perceived barriers and effective strategies to diabetes self-management. J Adv Nurs 2006;54(2):151-8.

136. Ahmed AT, Karter AJ, Warton EM, Doan JU, Weisner CM. The relationship between alcohol consumption and glycemic control among patients with diabetes: the Kaiser Permanente Northern California Diabetes Registry. J Gen Intern Med 2008;23(3):275-82.

137. Steenland K, Fine L. Shift work, shift change, and risk of death from heart disease at work. Am J Ind Med 1996;29(3):278-81.

138. Buell P, Breslow L. Mortality from coronary heart disease in California men who work long hours. J Chronic Dis 1960;11:615-26.

139. van der Hulst M. Long workhours and health Scandanavian Journal of Work Environmental Health 2003;29:171-88.

140. Landsbergis P. Long work hours, hypertension, and cardiovascular disease. Cad Saude Publica 2004;20(6):1746-8.

141. Sokejima S, Kagamimori S. Working hours as a risk factor for acute myocardial infarction in Japan: case-control study. Bmj 1998;317(7161):775-80.

142. Liu Y, Tanaka H. Overtime work, insufficient sleep, and risk of non-fatal acute myocardial infarction in Japanese men. Occup Environ Med 2002;59(7):447-51.

143. Trinkoff AM, Storr CL. Work schedule characteristics and substance use in nurses. Am J Ind Med 1998;34(3):266-71.

144. Shields M. Long working hours and health. Health Rep 1999;11(2):33-48. 
145. Mizoue T, Reijula K, Andersson K. Environmental tobacco smoke exposure and overtime work as risk factors for sick building syndrome in Japan. Am J Epidemiol 2001;154(9):803-8.

146. Yang H, Schnall PL, Jauregui M, Su TC, Baker D. Work hours and self-reported hypertension among working people in California. Hypertension 2006;48(4):744-50.

147. Centers for Disease Control and Prevention. Workplace Safety and Health. Department of Health and Human Services. National Institute for Occupational Safety and Health. Overtime and extended work shifts: Recent findings on illnesses, injuries, and health behaviors. 2004. Available from: http://www.cdc.gov/niosh/docs/2004-143/. (accessed August 2, 2009).

148. Hetrick R. Analyzing the recent upward surge in overtime hours. Monthly Labor Review 2000.

149. Rones PL, Ilg RE, JM G. Trends in hours of work since the mid-1970's. Monthly Labor Review 1997;120:3-10.

150. International Labour Office. Key indicators of the labour market 2001-2002. 2003. Palm Version. Routledge.Taylor and Francis NY.

151. Lockley SW, Landrigan CP, Barger LK, CA C. When policy meets physiology: the challenge of reducing resident work hours. Clinical Orthop 2006;449:116-27.

152. Caruso CC. Possible broad impacts of long work hours. Ind Health 2006;44(4):531-6.

153. Weijman I, Ros WJ, Rutten GE, Schaufeli WB, Schabracq MJ, Winnubst JA. The role of work-related and personal factors in diabetes self-management. Patient Educ Couns 2005;59(1):87-96.

154. Jabs J, Devine CM. Time scarcity and food choices: an overview. Appetite 2006;47(2):196-204.

155. Williamson AR, Hunt AE, Pope JF, Tolman NM. Recommendations of dietitians for overcoming barriers to dietary adherence in individuals with diabetes. Diabetes Educ 2000;26(2):272-9.

156. Gucciardi E, Demelo M, Offenheim A, Stewart DE. Factors contributing to attrition behavior in diabetes self-management programs: a mixed method approach. BMC Health Serv Res 2008;8:33.

157. Whitehouse FW, Whitehouse IJ, Smith J, Hohl RD. Teaching the person with diabetes: experience with a follow-up session. Diabetes Care 1979;2(1):35-8. 
158. Hammersley M, Holland M, Walford S, Thorn P. What happens to defaulters from a diabetic clinic? British Medical Journal 1985;9(291):1330-2.

159. Spikmans F, Brug J, Doven M, Kruizenga H, Hofsteenge G, van Bokhorst-van der Schueren M. Why do diabetic patients not attend appointments with their dietitian? Journal of Human Nutrition and Diet 2003;16(3):151-18.

160. Graziani C, Rosenthal M, Diamond J. Diabetes education program use and patient-perceived barriers to attendance. Family Medicine 1999;31(5):358-63.

161. Surwit RS, van Tilburg MA, Zucker N, McCaskill CC, Parekh P, Feinglos MN, et al. Stress management improves long-term glycemic control in type 2 diabetes. Diabetes Care 2002;25(1):30-4.

162. Brown AF, Ettner SL, Piette J, Weinberger M, Gregg E, Shapiro MF, et al. Socioeconomic position and health among persons with diabetes mellitus: a conceptual framework and review of the literature. Epidemiol Rev 2004;26:63-77.

163. Barglow P, Hatcher R, Edidin DV, Sloan-Rossiter D. Stress and metabolic control in diabetes: psychosomatic evidence and evaluation of methods. Psychosom Med $1984 ; 46(2): 127-44$.

164. Seeman TE, McEwen BS, Rowe JW, Singer BH. Allostatic load as a marker of cumulative biological risk: MacArthur studies of successful aging. Proc Natl Acad Sci U S A 2001;98(8):4770-5.

165. Surwit RS, Schneider MS. Role of stress in the etiology and treatment of diabetes mellitus. Psychosom Med 1993;55(4):380-93.

166. Kawakami N, Araki S, Ohtsu H, Hayashi T, Masumoto T, Yokoyama K. Effects of mood states, smoking and urinary catecholamine excretion on hemoglobin A1c in male Japanese workers. Ind Health 1995;33(4):153-62.

167. Wamala SP, Wolk A, Orth-Gomer K. Determinants of obesity in relation to socioeconomic status among middle-aged Swedish women. Prev Med 1997;26(5 Pt 1):734-44.

168. Halberg F. Some aspects of the chronobiology of nutrition: more work is needed on "when to eat". Journal of Nutrition 1989;119(3):333-43.

169. He X, Wharrad HJ. Diabetes knowledge and glycemic control among Chinese people with type 2 diabetes. Int Nurs Rev 2007;54(3):280-7. 
170. Moss R, Shoefer J. The crisis of physical illness: An overview of conceptual approach. In R. Moss (Ed.) Coping with physical illness 2: New perspectives. New York: Plenum. 1984.

171. Ingram M, Torres E, Redondo F, Bradford G, Wang C, O'Toole ML. The impact of promotoras on social support and glycemic control among members of a farmworker community on the US-Mexico border. Diabetes Educ 2007;33 Suppl 6:172S-178S.

172. McCann BS, Warnick GR, Knopp RH. Changes in plasma lipids and dietary intake accompanying shifts in perceived workload and stress. Psychosom Med 1990;52(1):97-108.

173. Marmot MG, Smith GD, Stansfeld S, Patel C, North F, Head J, et al. Health inequalities among British civil servants: the Whitehall II study. Lancet 1991;337(8754):1387-93.

174. Wickrama K, Conger RD, Lorenz FO. Work, marriage, lifestyle, and changes in men's physical health. J Behav Med 1995;18(2):97-111.

175. Morris DH, Sorensen G, Stoddard AM, Fitzgerald G. Comparison between food choices of working adults and dietary patterns recommended by the National Cancer Institute. J Am Diet Assoc 1992;92(10):1272-4.

176. Stewart B, Tinsley A. Importance of food choice influences for working young adults. J Am Diet Assoc 1995;95(2):227-30.

177. Carranza SN, LeBaron S. Adherence among Mexican Americans with type 2 diabetes: behavioral attribution, social support, and poverty. Fam Med 2004;36(8):53940 .

178. Heuer L, Lausch C. Living with diabetes: perceptions of Hispanic migrant farmworkers. J Community Health Nurs 2006;23(1):49-64.

179. Gleeson-Kreig J, Bernal H, Woolley S. The role of social support in the selfmanagement of diabetes mellitus among a Hispanic population. Public Health Nurs 2002;19(3):215-22.

180. Arcury TA, Quandt SA. Delivery of health services to migrant and seasonal farmworkers. Annu Rev Public Health 2007;28:345-63.

181. Tsuda K, Tsutsumi A, Kawakami N. Work-related factors associated with visiting a doctor for a medical diagnosis after a worksite screening for diabetes mellitus in Japanese male employees. J Occup Health 2004;46(5):374-81. 
182. Henry RR, Gumbiner B, Ditzler T, Wallace P, Lyon R, Glauber HS. Intensive conventional insulin therapy for type II diabetes. Metabolic effects during a 6-mo outpatient trial. Diabetes Care 1993;16(1):21-31.

183. Carver C. Insulin treatment and the problem of weight gain in type 2 diabetes. Diabetes Educ 2006;32(6):910-7.

184. Lindstrom T, Eriksson P, Olsson AG, Arnqvist HJ. Long-term improvement of glycemic control by insulin treatment in NIDDM patients with secondary failure. Diabetes Care 1994;17(7):719-21.

185. Weight gain associated with intensive therapy in the diabetes control and complications trial. The DCCT Research Group. Diabetes Care 1988;11(7):567-73.

186. Dey J, Blonde L, Guthrie R. Factors influencing patient acceptability of diabetes treatment regimens. Clinical Diabetes 2000;18(2).

187. Mann JI. Evidence-based nutrition recommendations for the treatment and prevention of type 2 diabetes and the metabolic syndrome. Food Nutr Bull 2006;27(2):161-6.

188. Barnoya J, Glantz SA. Cardiovascular effects of secondhand smoke: nearly as large as smoking. Circulation 2005;111(20):2684-98.

189. Panagiotakos DB, Pitsavos C, Chrysohoou C, Skoumas J, Masoura C, Toutouzas $\mathrm{P}$, et al. Effect of exposure to secondhand smoke on markers of inflammation: the ATTICA study. Am J Med 2004;116(3):145-50.

190. Henkin L, Zaccaro D, Haffner S, Karter A, Rewers M, Sholinsky P, et al. Cigarette smoking, environmental tobacco smoke exposure and insulin sensitivity: the Insulin Resistance Atherosclerosis Study. Ann Epidemiol 1999;9(5):290-6.

191. Rimm EB, Chan J, Stampfer MJ, Colditz GA, Willett WC. Prospective study of cigarette smoking, alcohol use, and the risk of diabetes in men. Bmj 1995;310(6979):5559.

192. Kawakami N, Takatsuka N, Shimizu H, Ishibashi H. Effects of smoking on the incidence of non-insulin-dependent diabetes mellitus. Replication and extension in a Japanese cohort of male employees. Am J Epidemiol 1997;145(2):103-9.

193. Repace J. Exposure to Secondhand smoke. In: OH WR, Steinemann AC, Wallace LA, eds. Exposure Analyses. Boca Raton: CRC Press; 2007: 201-235.; 2007.

194. Polonsky WH. Understanding and assessing diabetes-specific quality of life. Diabetes Spectrum 2000;13:36-41. 
195. Unden AL, Elofsson S. Do different factors explain self-rated health in men and women? Gender medicine 2006;3(4):295-308.

196. Lehmkuhl H, Nabors L. Children with diabetes: Satisfaction with school support, illness perceptions and $\mathrm{HbA} 1 \mathrm{C}$ levels. Journal of Developmental and Physical Disabilities 2008;20(2):101-114.

197. Weijman I, Ros WJ, Rutten GE, Schaufeli WB, Schabracq MJ, Winnubst JA. Frequency and perceived burden of diabetes self-management activities in employees with insulin-treated diabetes: relationships with health outcomes. Diabetes Res Clin Pract 2005;68(1):56-64.

198. Wikblad K, Leksell J, Wibell L. Health-related quality of life in relation to metabolic control and late complications in patients with insulin dependent diabetes mellitus. Qual Life Res 1996;5(1):123-30.

199. Brown SL, Pope JF, Hunt AE, Tolman NM. Motivational strategies used by dietitians to counsel individuals with diabetes. Diabetes Educ 1998;24(3):313-8.

200. Savoca MR, Miller CK, Quandt SA. Profiles of people with type 2 diabetes mellitus: the extremes of glycemic control. Soc Sci Med 2004;58(12):2655-66.

201. Valdmanis V, Smith DW, Page MR. Productivity and economic burden associated with diabetes. Am J Public Health 2001;91(1):129-30.

202. Weinberger M, Kirkman S, Samsa GP, Cowper PA, Shortliffe A, Simel DL, et al. The Relationship between Glycemic Control and Health-Related Quality of Life in Patients with Non-Insulin-Dependent Diabetes Mellitus. Medicare care 1994;32(12):1173-1181.

203. Nerenz DR, Repasky DP, Whitehouse FW, Kahkonen DM. Ongoing assessment of health status in patients with diabetes mellitus. Med Care 1992;30(5 Suppl):MS11224.

204. Nyhlin KT. The fine balancing act of managing diabetes. Scand J Caring Sci 1991;5(4):187-94.

205. Van den Berghe G, Wilmer A, Milants I, Wouters PJ, Bouckaert B, Bruyninckx $\mathrm{F}$, et al. Intensive insulin therapy in mixed medical/surgical intensive care units: benefit versus harm. Diabetes 2006;55(11):3151-9.

206. Tamborlane WV, Ahern J. Implications and results of the Diabetes Control and Complications Trial. Pediatr Clin North Am 1997;44(2):285-300. 
207. Merbis MA, Snoek FJ, Kanc K, Heine RJ. Hypoglycaemia induces emotional disruption. Patient Educ Couns 1996;29(1):117-22.

208. Wild D, von Maltzahn R, Brohan E, Christensen T, Clauson P, Gonder-Frederick L. A critical review of the literature on fear of hypoglycemia in diabetes: Implications for diabetes management and patient education. Patient Educ Couns 2007;68(1):10-5.

209. American Diabetes Association. Hypoglycemia. 2009. Available from: http://www.diabetes.org/utils/printthispage.jsp?PageID=TYPE1DIABETES3_232936 (accessed April 25, 2009).

210. American Diabetes Association. Hyperglycemia. 2009. Available from: http://www/diabetes.org/utils/printthispage.jsp?PageID=Type1DIABETES3_232942 (accessed April 20, 2009).

211. Van der Does FE, De Neeling JN, Snoek FJ, Kostense PJ, Grootenhuis PA, Bouter LM, et al. Symptoms and well-being in relation to glycemic control in type II diabetes. Diabetes Care 1996;19(3):204-10.

212. Gavard JA, Lustman PJ, Clouse RE. Prevalence of depression in adults with diabetes. An epidemiological evaluation. Diabetes Care 1993;16(8):1167-78.

213. Peyrot M, Rubin RR. Levels and risks of depression and anxiety symptomatology among diabetic adults. Diabetes Care 1997;20(4):585-90.

214. De Groot M, Jacobs AM, Samson JA, Welch G. Glycemic control and major Depression in patients with type I and type II diabetes mellitus. Journal of Psychosom Res 1999;46:425-435.

215. Garay-Sevilla ME, Malacara JM, Gutierrez-Roa A, Gonzalez E. Denial of disease in Type 2 diabetes mellitus: its influence on metabolic control and associated factors. Diabet Med 1999;16(3):238-44.

216. Peel E, Parry O, Douglas M, Lawton J. Blood glucose self-monitoring in noninsulin-treated type 2 diabetes: a qualitative study of patients' perspectives. $\mathrm{Br} \mathrm{J}$ Gen Pract 2004;54(500):183-8.

217. Lustman PJ, Griffith LS, Clouse RE. Depression in Adults with Diabetes. Semin Clin Neuropsychiatry 1997;2(1):15-23.

218. Goldney RD, Phillips PJ, Fisher LJ, Wilson DH. Diabetes, depression, and quality of life: a population study. Diabetes Care 2004;27(5):1066-70. 
219. Redekop WK, Koopmanschap MA, Stolk RP, Rutten GE, Wolffenbuttel BH, Niessen LW. Health-related quality of life and treatment satisfaction in Dutch patients with type 2 diabetes. Diabetes Care 2002;25(3):458-63.

220. Pouwer F, Snoek FJ. Association between symptoms of depression and glycaemic control may be unstable across gender. Diabet Med 2001;18(7):595-8.

221. Samaras K, Hayward CS, Sullivan D, Kelly RP, Campbell LV. Effects of postmenopausal hormone replacement therapy on central abdominal fat, glycemic control, lipid metabolism, and vascular factors in type 2 diabetes: a prospective study. Diabetes Care 1999;22(9):1401-7.

222. Archer JS. NAMS/Solvay Resident Essay Award. Relationship between estrogen, serotonin, and depression. Menopause 1999;6(1):71-8.

223. Lloyd CE, Dyer PH, Barnett AH. Prevalence of symptoms of depression and anxiety in a diabetes clinic population. Diabet Med 2000;17(3):198-202.

224. Songer TJ, LaPorte RE, Dorman JS, Orchard TJ, Becker DJ, Drash AL. Employment spectrum of IDDM. Diabetes Care 1989;12(9):615-22.

225. Mayfield JA, Deb P, Whitecotton L. Work disability and diabetes. Diabetes Care 1999;22(7):1105-9.

226. Bastida E, Pagan JA. The impact of diabetes on adult employment and earnings of Mexican Americans: findings from a community based study. Health Econ 2002;11(5):403-13.

227. Von Korff M, Katon W, Lin EH, Simon G, Ciechanowski P, Ludman E, et al. Work disability among individuals with diabetes. Diabetes Care 2005;28(6):1326-32.

228. Kraut A, Walld R, Tate R, Mustard C. Impact of diabetes on employment and income in Manitoba, Canada. Diabetes Care 2001;24(1):64-8.

229. Turner S, Cherry N, Robinson J. Workplace exposures and employment patterns in adult onset asthmatics and diabetics. Occup Med 2005;55(4):287-91.

230. Glasgow RE, Anderson RM. In diabetes care, moving from compliance to adherence is not enough. Something entirely different is needed. Diabetes Care 1999;22(12):2090-2.

231. Kristensen TS. Sickness absence and work strain among Danish slaughterhouse workers: an analysis of absence from work regarded as coping behaviour. Soc Sci Med $1991 ; 32(1): 15-27$. 
232. Taylor PJ. Sickness Absence Resistance Trans. Soc. Occupational Medicine 1968;18:96-100.

233. Gu K, Cowie CC, Harris MI. Mortality in adults with and without diabetes in a national cohort of the U.S. population, 1971-1993. Diabetes Care 1998;21(7):1138-45.

234. Center for Disease Control and Prevention. National Center for Health Statistics. Division of vital statistics, National Vital Statistics Report. Volume 57, November 14, April 2009. Available from: http:www/cdc/gov/nchs/data/nvsr/nvsr57/nvsr57_14.pdf. (accessed May 29, 2009).

235. Saydah S, Tao M, Imperatore G, Gregg E. GHb level and subsequent mortality among adults in the U.S. Diabetes Care 2009;32(8):1440-6.

236. Katon WJ, Rutter C, Simon G, Lin EH, Ludman E, Ciechanowski P, et al. The association of comorbid depression with mortality in patients with type 2 diabetes.

Diabetes Care 2005;28(11):2668-72.

237. Khaw KT, Wareham N, Luben R, Bingham S, Oakes S, Welch A, et al. Glycated haemoglobin, diabetes, and mortality in men in Norfolk cohort of european prospective investigation of cancer and nutrition (EPIC-Norfolk). Bmj 2001;322(7277):15-8.

238. Inzucchi SE, Siegel MD. Glucose control in the ICU--how tight is too tight? N Engl J Med 2009;360(13):1346-9.

239. Hoogwerf BJ. Does intensive therapy of type 2 diabetes help or harm? Seeking accord on ACCORD. Cleve Clin J Med 2008;75(10):729-37.

240. Soo Yeon S, Nesto RW. Implications of intensive glycemic control on cardiovascular disease: early reports from the ACCORD and ADVANCE Trials. Rev Cardiovasc Med 2008;9(1):1-4.

241. Cost-effectiveness of intensive glycemic control, intensified hypertension control, and serum cholesterol level reduction for type 2 diabetes. Jama 2002;287(19):2542-51.

242. Cefalu WT, Watson K. Intensive glycemic control and cardiovascular disease observations from the ACCORD study: now what can a clinician possibly think? Diabetes 2008;57(5):1163-5.

243. Goldman N, Lin IF, Weinstein M, Lin YH. Evaluating the quality of self-reports of hypertension and diabetes. J Clin Epidemiol 2003;56(2):148-54.

244. Koopman RJ, Mainous AG, 3rd, Diaz VA, Geesey ME. Changes in age at diagnosis of type 2 diabetes mellitus in the United States, 1988 to 2000. Ann Fam Med 2005;3(1):60-3. 
245. Turner R, Stratton I, Horton V, Manley S, Zimmet P, Mackay IR, et al. UKPDS 25: autoantibodies to islet-cell cytoplasm and glutamic acid decarboxylase for prediction of insulin requirement in type 2 diabetes. UK Prospective Diabetes Study Group. Lancet 1997;350(9087):1288-93.

246. Clark PA. Type 2 diabetes in youth. J S C Med Assoc 2009;105(2):51-4.

247. Selvin E, Crainiceanu CM, Brancati FL, Coresh J. Short-term variability in measures of glycemia and implications for the classification of diabetes. Arch Intern Med 2007;167(14):1545-51.

248. Cohen RM, Franco RS, Joiner CH. Is poor glycemic control associated with reduced red blood cell lifespan? Diabetes Care 2004;27(4):1013-4.

249. Behan KJ. Cessation of menstruation improves the correlation of FPG to hemoglobin Alc in Caucasian women. Clin Lab Sci 2006;19(4):225-30.

250. Reider CR, Halter CA, Castelluccio PF, Oakes D, Nichols WC, Foroud T. Reliability of reported age at onset for Parkinson's disease. Mov Disord 2003;18(3):2759.

251. Rhee MK, Slocum W, Ziemer DC, Culler SD, Cook CB, El-Kebbi IM, et al. Patient adherence improves glycemic control. Diabetes Educ 2005;31(2):240-50.

252. Mehta SN, Volkening LK, Anderson BJ, Nansel T, Weissberg-Benchell J, Wysocki T, et al. Dietary behaviors predict glycemic control in youth with type 1 diabetes. Diabetes Care 2008;31(7):1318-20.

253. Evans JM, Newton RW, Ruta DA, MacDonald TM, Stevenson RJ, Morris AD. Frequency of blood glucose monitoring in relation to glycaemic control: observational study with diabetes database. Bmj 1999;319(7202):83-6.

254. Schillinger D, Grumbach K, Piette J, Wang F, Osmond D, Daher C, et al. Association of health literacy with diabetes outcomes. Jama 2002;288(4):475-82.

255. Rothman R, Malone R, Bryant B, Horlen C, DeWalt D, Pignone M. The relationship between literacy and glycemic control in a diabetes disease-management program. Diabetes Educ 2004;30(2):263-73.

256. Grylls WK, McKenzie JE, Horwath CC, Mann JI. Lifestyle factors associated with glycaemic control and body mass index in older adults with diabetes. Eur J Clin Nutr 2003;57(11):1386-93. 
257. Savoca MR, Miller CK, Ludwig DA. Food habits are related to glycemic control among people with type 2 diabetes mellitus. J Am Diet Assoc 2004;104(4):560-6.

258. Lloyd CE, Dyer PH, Lancashire RJ, Harris T, Daniels JE, Barnett AH. Association between stress and glycemic control in adults with type 1 (insulin-dependent) diabetes. Diabetes Care 1999;22(8):1278-83.

259. Paschalides C, Wearden AJ, Dunkerley R, Bundy C, Davies R, Dickens CM. The associations of anxiety, depression and personal illness representations with glycaemic control and health-related quality of life in patients with type 2 diabetes mellitus. J Psychosom Res 2004;57(6):557-64.

260. Cox DJ, Irvine A, Gonder-Frederick L, Nowacek G, Butterfield J. Fear of hypoglycemia: quantification, validation, and utilization. Diabetes Care 1987;10(5):61721.

261. Polonsky WH, Davis CL, Jacobson AM, Anderson BJ. Correlates of hypoglycemic fear in type I and type II diabetes mellitus. Health Psychol 1992;11(3):199-202.

262. Frier BM. How hypoglycaemia can affect the life of a person with diabetes. Diabetes Metab Res Rev 2008;24(2):87-92.

263. Geurts SA, Schufeli WB, Rutte CG. Absenteeism, turnover intention and inequity in the employment relationship Work and stress 1999;13(3):253-267.

264. Borritz M, Rugulies R, Christensen KB, Villadsen E, Kristensen TS. Burnout as a predictor of self-reported sickness absence among human service workers: prospective findings from three year follow up of the PUMA study. Occup Environ Med 2006;63(2):98-106

265. Lloyd-Jones DM, Martin DO, Larson MG, Levy D. Accuracy of death certificates for coding coronary heart disease as the cause of death. Ann Intern Med 1998;129(12):1020-6.

266. American Diabetes Association, Diabetes in the workplace. 2006. Available from: http://www.diabetes.org/communityprograms-and-localevents/diabetes-in-theworkplace.jsp (accessed November 20, 2008).

267. Leeds HS, Richards EP. Legal issues in accommodating the Americans with disabilities act to the diabetic worker. J Leg Med 2008;29(3):271-83.

268. Munir F, Leka S, Griffiths A. Dealing with self-management of chronic illness at work: predictors for self-disclosure. Soc Sci Med 2005;60(6):1397-407. 
269. Baanders AN, Andries F, Rijken PM, Dekker J. Work adjustments among the chronically ill. Int J Rehabil Res 2001;24(1):7-14.

270. Greene DS, Geroy GD. Diabetes and job performance: an empirical investigation. Diabetes Educ 1993;19(4):293-8.

271. Gold AE, MacLeod KM, Frier BM. Frequency of severe hypoglycemia in patients with type I diabetes with impaired awareness of hypoglycemia. Diabetes Care 1994;17(7):697-703.

272. Leckie AM, Graham MK, Grant JB, Ritchie PJ, Frier BM. Frequency, severity, and morbidity of hypoglycemia occurring in the workplace in people with insulin-treated diabetes. Diabetes Care 2005;28(6):1333-8.

273. Riddle MC, Rosenstock J, Gerich J. The treat-to-target trial: randomized addition of glargine or human NPH insulin to oral therapy of type 2 diabetic patients. Diabetes Care 2003;26(11):3080-6.

274. Centers for Disease Control and Prevention. National Center for Chronic Disease Prevention and Health Promotion. Fact Sheet. Diabetesatwork.org. 2009 [cited 2009 March 29]; Available from: http://www.cdc.gov/diabetes/pubs/factsheets/atwork.htm

275. Bartel A, Taubman P. Health and labor market success: the role of various diseases. Rev Econ Stat 1979;61(1):1-8.

276. D'Arrigo T. The shift-work shuffle. Working irregular hours doesn't have to mean battling irregular blood glucose. Diabetes Forecast 2007;60(7):50-2.

277. D'Arrigo T. Man of steel. Working crazy hours in a steel mill gave Ed Stabile crazy blood glucose to match--until he made a few changes. Diabetes Forecast 2007;60(7):53-4.

278. DeWitt DE, Dugdale DC. Using new insulin strategies in the outpatient treatment of diabetes: clinical applications. Jama 2003;289(17):2265-9.

279. Samann A, Muhlhauser I, Bender R, Kloos C, Muller UA. Glycaemic control and severe hypoglycaemia following training in flexible, intensive insulin therapy to enable dietary freedom in people with type 1 diabetes: a prospective implementation study. Diabetologia 2005;48(10):1965-70.

280. Devine CM, Connors MM, Sobal J, Bisogni CA. Sandwiching it in: spillover of work onto food choices and family roles in low- and moderate-income urban households. Soc Sci Med 2003;56(3):617-30. 
281. Lowe J, Linjawi S, Mensch M, James K, Attia J. Flexible eating and flexible insulin dosing in patients with diabetes: Results of an intensive self-management course. Diabetes Res Clin Pract 2008;80(3):439-43.

282. Watkins KW, Connell CM, Fitzgerald JT, Klem L, Hickey T, Ingersoll-Dayton B. Effect of adults' self-regulation of diabetes on quality-of-life outcomes. Diabetes Care 2000;23(10):1511-5.

283. Grey M, Boland EA, Davidson M, Yu C, Tamborlane WV. Coping skills training for youths with diabetes on intensive therapy. Appl Nurs Res 1999;12(1):3-12.

284. Lustman PJ, Griffith LS, Freedland KE, Kissel SS, Clouse RE. Cognitive behavior therapy for depression in type 2 diabetes mellitus. A randomized, controlled trial. Ann Intern Med 1998;129(8):613-21.

285. Anderson RM, Funnell MM, Butler PM, Arnold MS, Fitzgerald JT, Feste CC. Patient empowerment. Results of a randomized controlled trial. Diabetes Care 1995;18(7):943-9.

286. Peyrot M, Rubin RR, Lauritzen T, Snoek FJ, Matthews DR, Skovlund SE. Psychosocial problems and barriers to improved diabetes management: results of the Cross-National Diabetes Attitudes, Wishes and Needs (DAWN) Study. Diabet Med 2005;22(10):1379-85.

287. Quintiliani L, Sattelmair J, Sorensen G. The workplace as a setting for interventions to improve diet and promote physical activity. Background paper prepared for the WHO/WEF Joint Event on Preventing Noncommunicable Diseases in the Workplace (Dalian/China, September 2007). 2007; Available from: http:///www.cdc.gov/niosh/worklife/steps/pdfs/NIOSH\%20integration\%20ms_postsymp\%20revision trckd\%20done.pdf (accessed November 10, 2009).

288. CDC. Healthy People 2010. 2nd ed. With Understanding and Improving Health and Objectives for Improving Health. 2 vols.: U.S. Government Printing Office; 2000.

289. Grosch JW, Alterman T, Petersen MR, Murphy LR. Worksite health promotion programs in the U.S.: factors associated with availability and participation. Am J Health Promot 1998;13(1):36-45.

290. Glasgow RE, McCaul KD, Fisher KJ. Participation in worksite health promotion: a critique of the literature and recommendations for future practice. Health Educ Q 1993;20(3):391-408.

291. Morris WR, Conrad KM, Marcantonio RJ, Marks BA, Ribisl KM. Do blue-collar workers perceive the worksite health climate differently than white-collar workers? Am J Health Promot 1999;13(6):319-24. 
292. Alexy B. Workplace health promotion and the blue collar worker. Aaohn J 1990;38(1):12-6.

293. Arent $\mathrm{S}$. The role of diabetes healthcare professionals in diabetes discrimination issues at work and school. Diabetes Educ 2002;28(6):1021-7. 


\section{EVELYN PATRICIA DAVILA}

$\begin{array}{ll}\text { April } 2001 & \begin{array}{l}\text { Bachelor of Science in Food and Nutrition } \\ \text { The Florida State University } \\ \text { Tallahassee, Florida }\end{array} \\ \text { WIC Nutrition Educator } \\ \text { Miami-Dade County Health Department } \\ \text { Miami, Florida }\end{array}$

\section{PUBLICATIONS}

Trepka MJ, Newman F, Davila EP, Matthew KJ, Dixon Z, Huffman FG.. Randomized Controlled Trial To determine the Effectiveness of an Interactive Multimedia Food Safety Program for WIC (Women, Infants, and Children) clients. Journal of the American Dietetic Association 2008; Jun 108 (6): 978-84.

Arheart KL, Lee DJ, Fleming LE, LeBlanc WG, Dietz NA, McCollister KE, Wilkinson JD, Lewis JE, Clark JD, Davila EP, Bandiera FC. Accuracy of self-reported smoking and secondhand smoke exposure in the US workforce: the National Health and Nutrition 
Examination Surveys. Journal of Occupational and Environmental Medicine 2008; 50 (12): 1414-1420.

Davila EP, Hlaing WM. Comorbidities of Patients with Hypertension Admitted to Emergency Departments in Florida Hospitals. Florida Public Health Review 2008; 5, 8492.

Vidal L, LeBlanc WG, McCollister KE, Arheart KL, Chung Bridges K, Christ S, Caban Martinez AJ, Lewis J, Lee DJ, Clark J, Davila EP, Fleming LE. Cancer Screening in US Workers. American Journal of Public Health 2009; 99(1): 59-65.

Lee DJ, Lam BL, Arora S, Arheart KL, McCollister KE, Zheng DD, Christ SL, Davila EP. Reported Eye care Utilization among US Adults: The 1997-2005 National Health Interview Survey. Archives of Ophthalmology 2009;127(3):303-10.

Davila EP, Caban-Martinez AJ, Fleming LE, Lam BL, Arheart KL, McCollister KE, LeBlanc WG, Zheng DD, Christ SL, Muennig P, Ferraro KF. Sensory Impairment in Older US Workers. American Journal of Public Health 2009; Aug;99(8):1378-85.

Lee DJ, Arheart KL, Lam BL, Zheng DD, Christ SL, McCollister KE, Davila EP. Trends in Reported Visual Impairment in United States Adults. Ophthalmic Epidemiology 2009; 16: 42-9.

Lam BL, Lee DJ, Zheng DD, Davila EP, Christ SL, Arheart KL. Disparity in Prevalence of Self-Reported Visual Impairment in Older Adults Among US Race-Ethnic Subgroups Ophthalmic Epidemiology 2009; 16: 144-50.

Davila EP, Zhao W, Byrne MM, Webb MS, Huang Y, Arheart K, Dietz N, CabanMartinez AJ, Parker DF, Lee DJ. Correlates of Smoking Quit Attempts: Florida Tobacco Callback Survey, 2007. Tobacco Induced Diseases 2009; Jun 29:5-10.

Davila EP, Trepka MJ, Newman FL, Huffman FG, Dixon Z. Diarrheal illness among Women, Infants, and Children (WIC) Program participants, Miami, Florida: Implications for nutrition education. Journal Nutrition Education and Behavior 2009; 41 (6):420-4.

Davila EP, Lee DJ, Fleming LE, LeBlanc WG, Arheart K, Dietz N, Lewis JE, McCollister K, Caban-Martinez A, Bandiera F. Sleep Disorders and Secondhand Smoke Exposure in the US Population. Nicotine and Tobacco Research 2010; 12 (3): 294-9.

Caban-Martinez AJ, Lee DJ, Davila EP, LeBlanc WG, Arheart KL, Dietz N, Lewis JE, McCollister K, Caban-Martinez A, Bandiera F. Sustained low influenza vaccination rates in US healthcare workers. Preventive Medicine 2010; 50 (4):210-212. 